\title{
Dynamic Characteristics of High-speed Water- lubricated Spiral Groove Thrust Bearing based on Turbulent Cavitating Flow Lubrication Model
}

Xiaohui Lin ( $\sim$ Lxh60@seu.edu.cn )

Southeast University https://orcid.org/0000-0001-7849-5734

shun Wang

Southeast University

shuyun Jiang

Southeast University

shaowen Zhang

Southeast University

Original Article

Keywords: spiral groove thrust bearings, water lubrication, turbulent lubrication, cavitating flow, dynamic characteristics

Posted Date: May 29th, 2020

DOI: https://doi.org/10.21203/rs.3.rs-31639/v1

License: (c) (i) This work is licensed under a Creative Commons Attribution 4.0 International License. Read Full License

Version of Record: A version of this preprint was published at Chinese Journal of Mechanical Engineering on February 22nd, 2022. See the published version at https://doi.org/10.1186/s10033-021-00671-3. 


\section{Title page}

\section{Dynamic Characteristics of High-speed Water-lubricated Spiral Groove Thrust Bearing based on Turbulent Cavitating Flow Lubrication Model}

Xiaohui Lin, born in 1960, is currently an associate professor at School of Mechanical Engineering, Southeast University, China.He received his bachelor degree from Wuhan University of Technology, China, in 1982. His research interests include two-phase flow transport theory. born in 1960,Associate Professor of School of Mechanical Engineering, Southeast University.

Tel: 13851760077; E-mail: Lxh60@seu.edu.cn

Shun Wang, born in 1995, is currently a master at Southeast University, China.

Tel: 18251985252; E-mail: 220170310@seu.edu.cn

Shuyun Jiang, born in 1966, is currently a PhD supervisor at Southeast University, China.

Tel: 13605161056; E-mail: jiangshy@seu.edu.cn

Shaowen Zhang, is currently $\mathrm{PhD}$ at Southeast University, China.

Tel: 15051867239; E-mail: shaowen2004@163.com

Corresponding author: Xiaohui Lin E-mail: Lxh60@seu.edu.cn 


\title{
ORIGINAL ARTICLE
}

\section{Dynamic Characteristics of High-speed Water-lubricated Spiral Groove Thrust Bearing based on Turbulent Cavitating Flow Lubrication Model}

\author{
Xiaohui Lin ${ }^{1}$, Shun Wang, Shuyun Jiang, Shaowen Zhang \\ School of Mechanical Engineering Southeast University, Nanjing 211189, China
}

\begin{abstract}
In this paper, a turbulent cavitating flow lubrication model based on twophase fluid and population balance equation of bubbles was established to analyze dynamic characteristics of high speed water-lubricated spiral groove thrust bearings(SGTB). Stiffness and the damping coefficients of the SGTB were calculated using the perturbation pressure equations. An experimental apparatus was developed to verify the theoretical model. Simulating and experimental results show that the smallsized bubbles tend to generate in the turbulent cavitating flow when at a high rotary speed, and the bubbles mainly locate at the edges of the spiral groove. The simulating results also show that the direct stiffness coefficients are increased due to cavitation effect, and cross stiffness coefficients and damping coefficients are hardly affected by the cavitation effect. Turbulent effect on the dynamic characteristics of SGTB is much stronger than the cavitating effect.
\end{abstract}

Keywords: spiral groove thrust bearings; water lubrication; turbulent lubrication; cavitating flow; dynamic characteristics

\section{Introduction}

Water-lubricated bearing has been widely applied due to its low viscosity, low temperature rise, no pollution and etc. Spiral groove thrust bearing (SGTB) has advantages in hydrodynamic effect and stability. Nowadays, the water-lubricated spiral groove thrust bearing has been used in high-speed spindle. However, since inception cavitation number of water is larger than that of oil, cavitation phenomenon in waterlubricated bearings is more serious than the oil-lubricated bearing at high speed, and the water-lubricated bearing tends to fall into turbulent fluid condition when rotary speed exceeds a certain value. Hence, both cavitating effect and turbulent effect should be considered to model the high-speed water-lubricated bearing.

The cavitating and turbulent effects of water or oil bearings with different 
configurations have been investigated in previous studies. In the early studies on bearing cavitation, the different boundary conditions for lubrication equation including the Swift-Stiebe condition [1], the JFO condition [2], the Elrod condition [3] have been adopted to describe the cavitation effect of the lubricating film. With those conditions, solution of a two-phase flow in cavitated region can be avoided. However, the cavitating flow is not involved in those models. To overcome this problem, the cavitation lubricant is treated as two-phase mixed fluid. The two-phase mixed fluid models have been developed, those models can be divided into three types based on calculation approaches of gas phase volume fraction: (1) the model based on the R-P equation [4,5] (2) the model based on gas solubility and surface tension of bubble [4,6-9] (3) the model based on transport equation of the gas phase volume fraction [10-15]. In addition, with the rapid development of computational fluid dynamics (CFD) technology, some researchers have proposed performance analysis of bearings with the cavitation using the CFD software [7,11,16-19]. However, the interfacial effect of the two-phase flow is not included in the above lubrication models of cavitating flow. Actually, the momentum and energy exchange between the two phases produces at the interface, so the interface effect is a very important feature for two-phase flow, which can not be ignored when modeling the high-speed water-lubricated bearing. In addition, the size distribution of the bubbles cannot be obtained using the present mixed fluid model or the CFD model. In our previous study, lubrication models considering the interface effect of two-phase flow have been proposed[20-23]. However, those models are established under heat isolation, and the bubble population equilibrium equation is solved directly. Since the bubble size probability density function is an unknown quantity, so it is difficult to determine the bubble size probability density function at the initial moment and at the interface. Furthermore, this kind of algorithm tends to unstable.

This study aims to establish a lubrication model for the water-lubricated spiral groove thrust bearings including both two-phase interfacial and fluid turbulent and bearing heat conduction effects. A generalized Reynolds equation considering the cavitation interface and turbulence, and an energy equation considering bearing heat conduction are derived. A population balance equation considering the breakage and coalescence of bubbles is introduced to describe the evolution of the size distribution of bubbles. A discrete interval method is employed to solve the bubble population balance equation. The influence of cavitation and turbulence on the dynamic characteristics of the SGTB 
is analyzed using the established model. The size distribution of cavitation bubbles and stiffness coefficient of water film are measured using a self-developed experimental device. A comparison of the simulating results with experimental ones is given to verify the proposed model.

\section{Theoretical model}

\subsection{The generalized Reynolds equation based on the two-phase flow theory}

This paper studies the water-lubricated SGTB with the turbulent cavitating flow film. The classical Reynolds equation is not suitable for describing the bearing lubrication state, a Reynolds equation which contains turbulent and two-phase interfacial effects is needed, so the following basic assumptions are made for the modeling:

The bubbles in the cavitation flow be regarded as spheres.

The flow field is a small perturbation of the turbulent Couette flow [24].

The turbulent shear stress is defined by the law of wall, Reichardt [25] empirical formula is used to define the eddy viscosity coefficient.

The average viscosity along the film thickness is adopted in generalized turbulent Reynolds equation.

The spiral area is an irregular area in the polar coordinate system, a spiral coordinates system $(\eta, \zeta)$ are adopted to improve numerical calculation accuracy. The polar coordinates $(\theta, r)$ are converted into the spiral coordinates system $(\eta, \zeta)$ as follows:

$$
\begin{gathered}
\zeta=r \\
\eta=\theta-f(\zeta) \\
f(\zeta)=\frac{1}{\cot \beta} \ln \left(\frac{\zeta}{\zeta_{b}}\right)
\end{gathered}
$$

The spiral patterns before and after the coordinates conversion are shown in Fig. 2. 


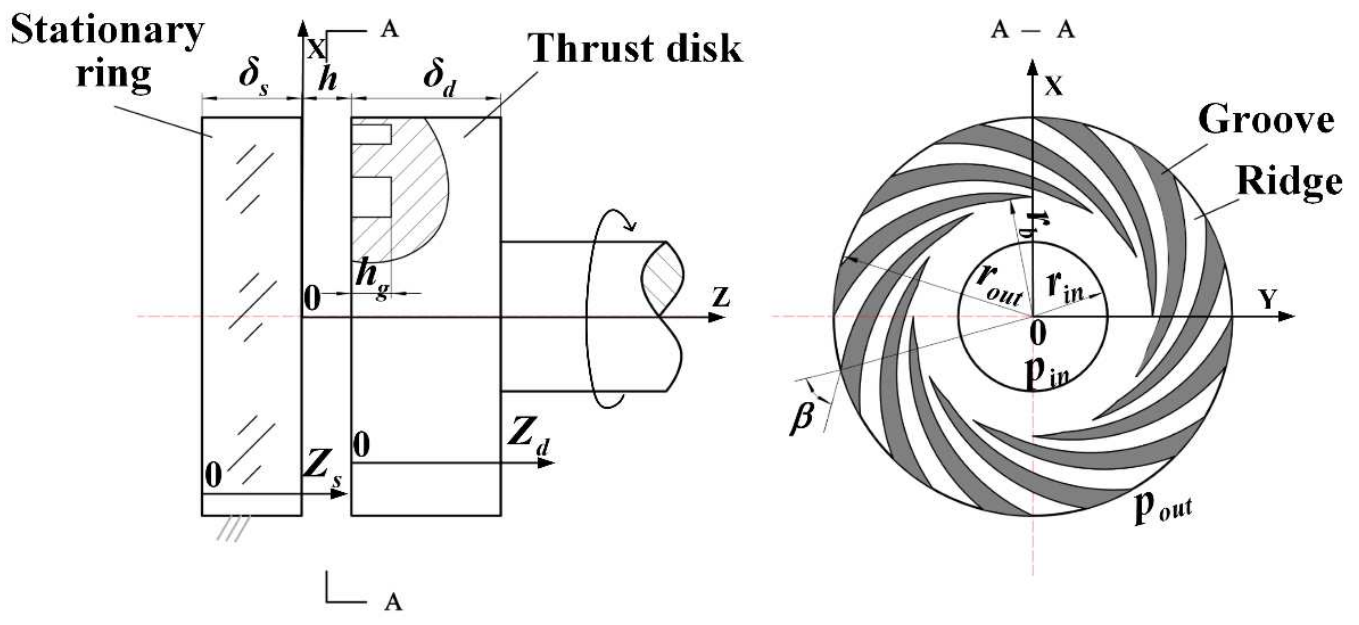

Fig. 1 Structure schematic of inward-pumping SGTB
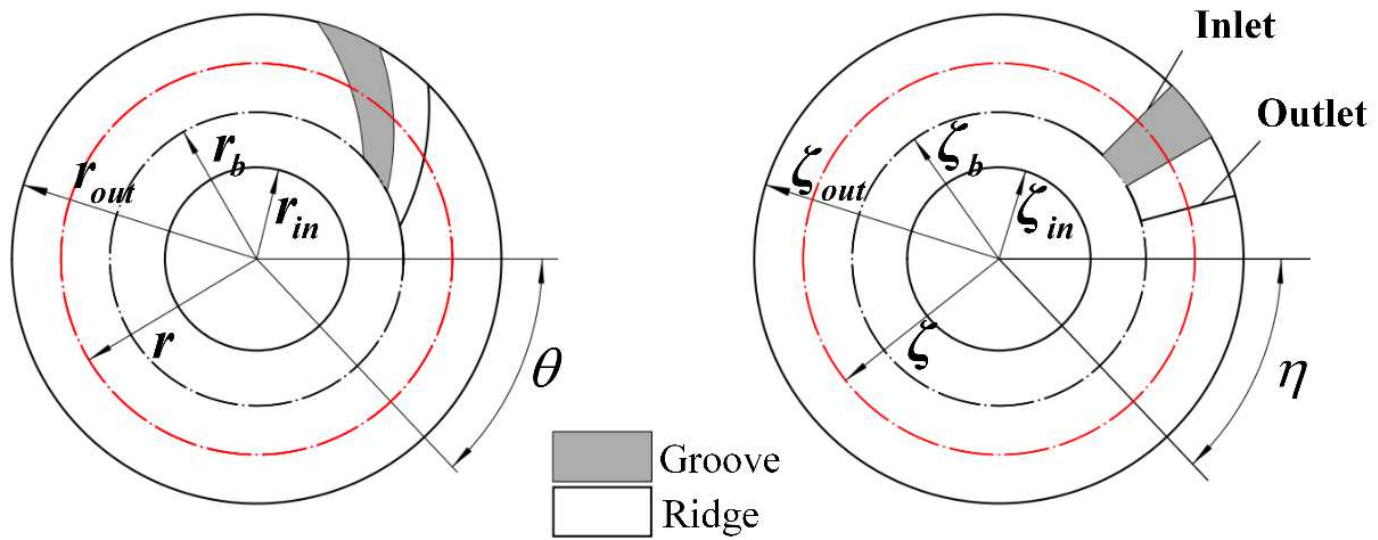

Fig. 2 Coordinates transformation of spiral patterns

A generalized turbulent Reynolds equation with cavitation interface effects and inertial effects in the $(\eta, \zeta)$ coordinates is derived based on two-phase flow theory[26]

$$
\begin{gathered}
\frac{\partial}{\partial \zeta}\left(\frac{h^{3} \zeta}{\bar{\mu}_{w} k_{r}} \frac{\partial C_{w} p}{\partial \zeta}\right)+\frac{\partial}{\partial \eta}\left(\left(\frac{\zeta^{2} f^{\prime 2}(\zeta)}{k_{r}}+\frac{1}{k_{\theta}}\right) \frac{h^{3}}{\bar{\mu}_{w} \zeta} \frac{\partial C_{w} p}{\partial \eta}\right) \\
-\frac{\partial}{\partial \zeta}\left(\frac{h^{3} \zeta}{\bar{\mu}_{w} k_{r}} f^{\prime}(\zeta) \frac{\partial C_{w} p}{\partial \eta}\right)-\frac{\partial}{\partial \eta}\left(\frac{h^{3} \zeta}{\bar{\mu}_{w} k_{r}} f^{\prime}(\zeta) \frac{\partial C_{w} p}{\partial \zeta}\right) \\
=\frac{\partial}{\partial \eta}\left(\frac{h U C_{w}}{2}\right)+\frac{\partial}{\partial \eta}\left(\frac{h^{3}}{\bar{\mu}_{w} k_{\theta}} M(\eta)\right)+ \\
\frac{\partial}{\partial \zeta}\left(\frac{h^{3} \rho_{w} \bar{u}^{2} C_{w}}{\bar{\mu}_{w} k_{r}}\right)-\frac{\partial}{\partial \eta}\left(f^{\prime}(\zeta) \frac{h^{3} \rho_{w} \bar{u}^{2} C_{w}}{\bar{\mu}_{w} k_{r}}\right)
\end{gathered}
$$

where

$$
\begin{gathered}
\bar{\mu}_{w}=\frac{1}{h} \int_{0}^{h} \mu_{w} d z \\
\bar{u}=\frac{1}{h} \int_{0}^{h} u u d z
\end{gathered}
$$




$$
\begin{gathered}
\frac{1}{k_{\theta}}=\int_{0}^{1} \int_{0}^{z^{*}} \frac{1}{f_{c}}\left(1-\frac{g_{c}}{f_{c}}\right)\left(\frac{1}{2}-z^{* \prime}\right) d z^{* \prime} d z^{*} \\
\frac{1}{k_{r}}=\int_{0}^{1} \int_{0}^{z^{*}} \frac{1}{f_{c}}\left(\frac{1}{2}-z^{* \prime}\right) d z^{* \prime} d z^{*} \\
f_{c}=0.4\left[z^{*} h_{c}^{*}-10.7 \operatorname{th}\left(\frac{z^{*} h_{c}^{*}}{10.7}\right)\right]+1 \\
g_{c}=0.2 z^{*} h_{c}^{*} \operatorname{th} h^{2}\left(\frac{z^{*} h_{c}^{*}}{10.7}\right) \\
z^{*}=\frac{z}{h} \\
h_{c}^{*}=\frac{h}{v_{w}} \sqrt{\frac{\tau_{c}}{\rho_{w}}}
\end{gathered}
$$

The following expression can be given for the logarithmic spirals

$$
f^{\prime}(\zeta)=\frac{1}{\zeta \cot \beta}
$$

The circumferential velocity distribution of the water film is written as

$$
u=\frac{U}{2}+\frac{\left(h_{c}^{*}\right)^{2}}{R_{c}} \frac{U}{h^{*}} G_{1}\left(z^{*}\right)-\frac{1}{C_{w}} \frac{h^{2}}{\bar{\mu}_{w}}\left(\frac{1}{\zeta} \frac{\partial\left(C_{w} p\right)}{\partial \eta}-M(\eta)\right) G_{2}\left(z^{*}\right)
$$

where

$$
\begin{gathered}
G_{1}\left(z^{*}\right)=\int_{\frac{1}{2}}^{z^{*}} \frac{1}{f_{c}} d z^{* \prime} \\
G_{2}\left(z^{*}\right)=\int_{0}^{z^{*}} \frac{1}{f_{c}}\left(1-\frac{g_{c}}{f_{c}}\right) d z^{* \prime} \\
C_{w}=1-C_{g} \\
C_{g}=\frac{\frac{\pi}{6} \int_{0}^{\infty} \varsigma^{3} f_{e q}(\eta, \varsigma) d \varsigma}{1+\frac{\pi}{6} \int_{0}^{\infty} \varsigma 3 f_{e q}(\eta, \varsigma) d \varsigma}
\end{gathered}
$$

where, $f_{e q}(\eta, \varsigma)$ is the equilibrium distribution function of the cavitation bubble diameter; $f_{e q}(\eta, \varsigma) d \varsigma$ represents the number of bubbles with diameter between $(\varsigma, \varsigma+d \varsigma)$ per unit volume of water at spatial coordinate $\eta$ under the equilibrium state. The first term on the right-hand side of Eq. (2) is the hydrodynamic effect, the second term on the right side of Eq. (2) is the interface hydrodynamic effect, the third and fourth terms on the right side of Eq. (2) is the inertia hydrodynamic effect. The interface momentum transfer function includes the following three parts:

$$
M(\eta)=M_{1}(\eta)+M_{2}(\eta)+M_{3}(\eta)
$$

where, $M_{1}(\eta)$ is the interface momentum transfer term due to mass transfer, $M_{2}(\eta)$ is the interface momentum transfer term due to viscous drag, $M_{3}(\eta)$ is the interface 
momentum transfer term due to surface tension. Because the film thickness is thin, the liquid velocity in $M_{1}(\eta), M_{2}(\eta)$ are approximated by the average velocity. $M_{1}(\eta), M_{2}(\eta)$ and $M_{3}(\eta)$ are written as:

$$
\begin{gathered}
M_{1}=-\left[\rho_{w} \frac{\pi}{3} \int_{0}^{\infty} \varsigma^{2} f_{e q}(\eta, \varsigma) d \varsigma\right] \bar{u}\left(\bar{u}-u_{b}\right) \\
M_{2}=\left[\frac{\pi}{3} C_{D s} \rho_{w} \int_{0}^{\infty} \varsigma^{2} f_{e q}(\eta, \varsigma) d \varsigma\right]\left(\bar{u}-u_{b}\right)^{2} \\
M_{3}=2 \pi \sigma \int_{0}^{\infty} \varsigma f_{e q}(\eta, \varsigma) d \varsigma
\end{gathered}
$$

where $C_{D S}=\frac{24}{R_{e}}$

Assume that the range of bubble diameter is divided into $\mathrm{M}$ smaller intervals, the diameter distribution of the bubbles is uniform in a smaller interval. So the equilibrium distribution function of bubble diameter in the kth smaller diameter interval $\left(\varsigma_{k}, \varsigma_{k+1}\right)$ can be expressed as.

$$
f_{e q}(\eta, \varsigma) \approx n_{e q}^{k}(\eta) \delta\left(\varsigma-x_{k}\right)
$$

where

$$
\begin{aligned}
n_{e q}^{k}(\eta) & =\int_{\varsigma_{k}}^{\varsigma_{k+1}} f_{e q}(\eta, \varsigma) d \varsigma \\
x_{k} & =\frac{1}{2}\left(\varsigma_{k}+\varsigma_{k+1}\right)
\end{aligned}
$$

where $n_{e q}^{k}(\eta)$ is the number of bubbles in the $k$ th diameter interval $\left(\varsigma_{k}, \varsigma_{k+1}\right)$. And the integral in Eq. (12) can be approximated as

$$
\begin{aligned}
\int_{0}^{\infty} \varsigma^{2} f_{e q}(\eta, \varsigma) d \varsigma & \approx \sum_{k=1}^{M} x_{k}^{2} n_{e q}^{k}(\eta) \\
\int_{0}^{\infty} \varsigma f_{e q}(\eta, \varsigma) d \varsigma & \approx \sum_{k=1}^{M} x_{k} n_{e q}^{k}(\eta)
\end{aligned}
$$

The boundary conditions of Eq. (2) are 


$$
\begin{gathered}
\left.p\right|_{\zeta=\zeta_{\text {out }}}=0 \\
\left.p\right|_{\zeta=\zeta_{\text {in }}}=p_{\text {in }} \\
p(\eta)=p\left(\eta+\frac{2 \pi}{N}\right)
\end{gathered}
$$

The pressure at the groove-ridge boundary can be solved by using continuous condition of flow at the groove-ridge boundary. The finite volume at the groove-ridge boundary is shown in Fig. 3.

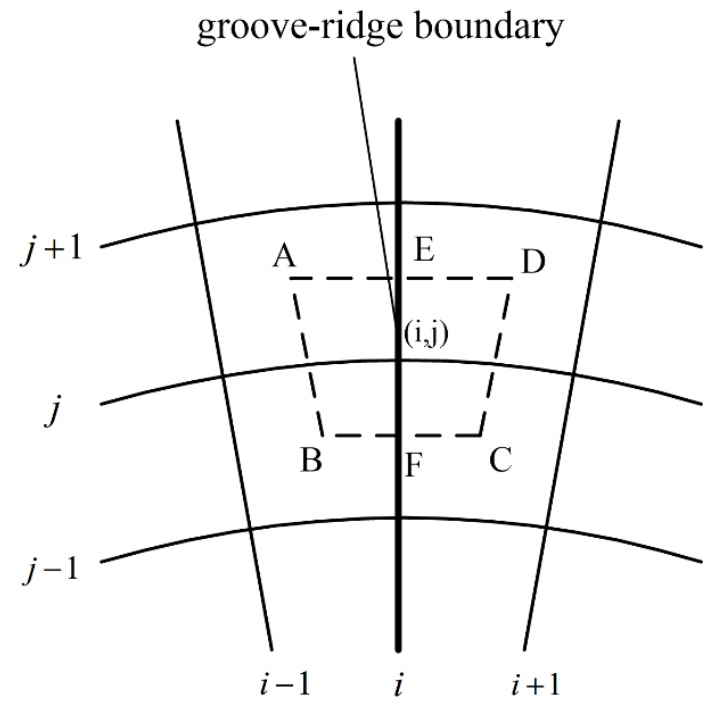

Fig.3 Finite volume separated by groove-ridge boundary

In a finite volume at the groove-ridge boundary, the continuous condition of the flow can be written as

$$
\sum_{i} Q_{i}^{\eta}+\sum_{j} Q_{j}^{\zeta}=0
$$

where

$$
\begin{gathered}
Q_{i}^{\eta}=\int_{\zeta_{1}}^{\zeta_{2}}\left(\begin{array}{c}
\zeta f^{\prime}(\zeta)\left(\frac{h^{3}}{C_{w} \mu_{m}}\left(\frac{\partial C_{w} p}{\partial \zeta}-\frac{1}{\zeta \cot \beta} \frac{\partial C_{w} p}{\partial \eta}\right)-\frac{h^{3}}{\mu_{w}} \frac{\rho \bar{u}^{2}}{\zeta}\right) \frac{1}{k_{r}} \\
+\frac{U h}{2}-\frac{h^{3}}{\mu_{m}}\left(\frac{1}{\zeta C_{w}} \frac{\partial C_{w} p}{\partial \eta}-\frac{M(\eta)}{C_{w}}\right) \frac{1}{k_{\theta}}
\end{array}\right) d \zeta \\
(i=B F, F C, D E, E A) \\
Q_{j}^{\zeta}=\int_{\eta_{1}}^{\eta_{2}} \zeta\left(-\frac{h^{3}}{C_{w} \mu_{m}}\left(\frac{\partial C_{w} p}{\partial \zeta}-f^{\prime}(\zeta) \frac{\partial C_{w} p}{\partial \eta}\right) \frac{1}{k_{\theta}}+\frac{h^{3}}{\mu_{m}} \frac{\rho \bar{u}^{2}}{\zeta} \frac{1}{k_{r}}\right) d \eta \\
(j=A B, C D)
\end{gathered}
$$

\subsection{Turbulent cavitating flow energy equation}

The temperature field of the cavitating flow water film can be obtained by solving the two-phase flow energy equation under high-speed conditions, and the following assumptions are adopted to simplify the energy equation. 
(1) The circumferential convection term is only considered in the energy equation, because the circumferential velocity is much larger than the radial velocity.

(2) Turbulent pulsation heat conduction term is ignored.

(3) The viscous dissipation term along the film thickness is only considered in the energy equation.

According to the above assumption, the energy equation in the $(\eta, \zeta)$ coordinates in the spiral coordinate system can be simplified to

$$
\rho_{w} c_{v}\left(\frac{u}{\zeta} \frac{\partial\left(C_{w} T\right)}{\partial \eta}\right)=C_{w} k_{w} \frac{\partial^{2} T}{\partial z^{2}}+C_{w}\left(\tau_{t}(z)\right)\left(\frac{\partial u}{\partial z}\right)+E_{\text {int }}
$$

where

$$
\begin{aligned}
& \left(\tau_{t}(z)\right)=\frac{\left(h_{c}^{*}\right)^{2} \bar{\mu}_{w} U}{R_{c} h}+\frac{1}{C_{w}}\left(\frac{1}{\zeta} \frac{\partial\left(C_{w} p\right)}{\partial \eta}-M(\eta)\right)\left(z-\frac{h}{2}\right) \\
& \frac{\partial u}{\partial z}=\frac{\left(h_{c}^{*}\right)^{2}}{R_{c} h^{*}} \frac{\partial G_{1}\left(z^{*}\right)}{\partial z^{*}} \frac{U}{h}-\frac{h}{C_{w} \bar{\mu}_{w}}\left(\frac{1}{\zeta} \frac{\partial\left(C_{w} p\right)}{\partial \eta}-M(\eta)\right) \frac{\partial G_{2}\left(z^{*}\right)}{\partial z^{*}}
\end{aligned}
$$

The interface term $E_{\text {int }}$ in the energy equation is expressed as

$$
\begin{gathered}
E_{\text {int }} \approx C_{D s} \pi\left(\bar{u}-u_{b}\right)^{2} \bar{u} \sum_{k=1}^{M} x_{k}^{2} n_{e q}^{k}(\eta)-\rho_{w} c_{v} T\left(\bar{u}-u_{b}\right) \pi \sum_{k=1}^{M} x_{k}^{2} n_{e q}^{k}(\eta) \\
+2 \sigma \bar{u} \pi \sum_{k=1}^{M} x_{k} n_{e q}^{k}(\eta)
\end{gathered}
$$

The following boundary conditions are adopted for the energy Eq. (21)

(1) At the inlet of the spiral groove

$$
T(0, z)=T_{0}
$$

(2) On the interface of the water film and the thrust disk

$$
T(\eta, h)=T_{d}(\eta, 0)
$$

(3) On the interface of the water film and the stationary ring

$$
T(\eta, h)=T_{s}(\eta, \delta)
$$

The viscosity temperature relationship is written as

$$
\mu_{w}=\mu_{w 0} e^{\left(-b\left(T-T_{0}\right)\right)}
$$

The water film temperature field can be obtained solved by simultaneously solving the energy equation (21), the heat conduction equation of the stationary ring and the heat conduction equation of the thrust disk. The surface temperature $T_{d}(\eta, 0)$ of the stationary ring and surface temperature $T_{s}(\eta, \delta)$ of the thrust plate are set as the boundary condition of the energy equation (21).

\subsection{The heat conduction equation in stationary ring}


The circumferential and radial heat conduction of the stationary ring are ignored, the heat conduction equation is simplified as follows

$$
\frac{\partial^{2} T_{\mathrm{s}}}{\partial z_{S}^{2}}=0 \quad\left(0 \leq z_{S} \leq \delta_{S}\right)
$$

The boundary conditions of Eq. (29) are given as follows

1) At the surface between the stationary ring and the fluid $\left(z_{s}=\delta_{s}\right)$

$$
\begin{aligned}
-\left.k_{s} \frac{\partial T_{s}}{\partial z_{s}}\right|_{z_{s}=\delta_{s}} & =\alpha_{w}\left(\left.T_{s}\right|_{z_{s}=\delta_{s}}-T_{m}\right) \\
T_{m} & =\frac{1}{h} \int_{0}^{h} T d z
\end{aligned}
$$

2) At the surface between the stationary ring and the ambient $\left(z_{s}=0\right)$

$$
\left.k_{s} \frac{\partial T_{s}}{\partial z_{s}}\right|_{z_{S}=0}=\alpha_{a}\left(\left.T_{s}\right|_{z_{s}=0}-T_{0}\right)
$$

The expression of the temperature at the surface of the stationary ring can be obtained from Eq. (29) and boundary conditions (30), (31). The temperature at the surface of the stationary ring is written as

$$
\left.T_{s}\right|_{z_{s}=\delta_{s}}=\frac{T_{m}-T_{0}}{\frac{\alpha_{w}}{\alpha_{a}}-\frac{\alpha_{W}}{k_{s}} \delta_{s}-1}+T_{m}
$$

\subsection{The heat conduction equation in thrust disk}

For the rotary thrust disk, the heat transfer effects in circumferential and radial directions are also ignored, the heat conduction equation is simplified as follows

$$
\frac{k_{d}}{\rho_{d} c_{d}} \frac{\partial^{2} T_{d}}{\partial z_{d}^{2}}=\omega \frac{\partial T_{d}}{\partial \theta} \quad\left(0 \leq z_{d} \leq \delta_{d}\right)
$$

The boundary conditions of Eq. (33) are given as follows

1) At the surface between the thrust disk and the fluid $\left(z_{d}=0\right)$

$$
\left.k_{d} \frac{\partial T_{d}}{\partial z_{d}}\right|_{z_{r}=0}=\alpha_{w}\left(\left.T_{d}\right|_{z_{d}=0}-T_{m}\right)
$$

2) At the surface between the thrust disk and the ambient $\left(z_{d}=\delta_{d}\right)$

$$
\left.k_{d} \frac{\partial T_{d}}{\partial z_{d}}\right|_{z_{d}=\delta_{d}}=-\alpha_{a}\left(\left.T_{d}\right|_{z_{d}=\delta_{d}}-T_{0}\right)
$$

\subsection{The Force Balance Equation of Bubble}

The force balance equation of bubbles is expressed as

$$
F_{B}+F_{D}+F_{G}=0
$$

where 


$$
\begin{gathered}
F_{B}=\langle V\rangle\left(\rho_{w}-\rho_{g}\right) g \\
F_{G}=\left(\rho_{w}-\rho_{g}\right)\langle V\rangle \frac{d u}{d t} \\
\frac{d u}{d t}=-\frac{1}{\rho_{w}} \frac{\partial p}{\zeta \partial \eta} \\
\langle V\rangle \approx \frac{\pi}{6} \sum_{k=1}^{M} x_{k}^{3} n_{e q}^{k}(\eta) \\
F_{D}=12 \pi \bar{\mu}_{w}\left\langle R_{b}\right\rangle\left(u_{b}-\bar{u}\right) \frac{1-C_{g}^{\frac{5}{3}}}{\left(1-C_{g}\right)^{2}} \\
\left\langle R_{b}\right\rangle \approx \frac{1}{2} \sum_{k=1}^{M} x_{k} n_{e q}^{k}(\eta)
\end{gathered}
$$

The bubble velocity $u_{b}$ in the cavitating flow is calculated by the force balance Eq. (36). Substitute $F_{B}, F_{G}, F_{D}$ into Eq. (36), the bubble velocity $u_{b}$ can be expressed as [27]

$$
u_{b}=\bar{u}+\frac{\left(1-C_{g}\right)^{2}\left(\rho_{w}-\rho_{g}\right)}{12 \pi \bar{\mu}_{w}\left\langle R_{b}\right\rangle\left(1-C_{g}^{\frac{5}{3}}\right)}\langle V\rangle\left(g+\frac{1}{\rho_{w}} \frac{\partial p}{\zeta \partial \eta}\right)
$$

\subsection{The population balance equation of bubbles}

\subsubsection{The population balance equation}

Interface effect is an important phenomenon for cavitating flow, the momentum, mass and energy transfer occur through the interface between the gas-liquid. The interfacial area per unit volume liquid is positively correlated with the size distribution of the bubble, the bubble size distribution can be described by defining a probability density function $f(\mathbf{r}, \varsigma, t)$, and the internal coordinates $\varsigma$ are taken as bubble diameter in the model, and the $f(\mathbf{r}, \varsigma, t) d \varsigma$ represents the number of bubbles with between $(\varsigma, \varsigma+d \varsigma)$ at per volume liquid. Thus, integration of $f$ over bubble diameter results in the total number of bubbles per volume liquid. The breakage, coalescence are two main factors affecting bubble size distribution in the cavitating flow, and bubble size distribution is predicted by the model of the breakage and coalescence processes of bubbles, this leads to the so-called population balance equation (PBE).

The PBE is a transport equation, the evolution of function $f$ can be described by the PBE in the spiral coordinates $(\zeta, \eta)$, the PBE is written as follows 


$$
\begin{gathered}
\frac{\partial f(\eta, \varsigma)}{\partial t}+u_{b} \frac{\partial}{\zeta \partial \eta}(f(\eta, \varsigma))=-b(\varsigma) f(\eta, \varsigma) \\
+\int_{\varsigma}^{\varsigma_{\max }} h_{b}(\xi, \varsigma) b(\xi) f(\eta, \xi) d \xi-f(\eta, \varsigma) \int_{0}^{\varsigma_{\max }} c(\varsigma, \xi) f(\eta, \xi) d \xi \\
+\frac{\varsigma^{2}}{2} \int_{0}^{\varsigma} \frac{c\left(\left(\varsigma^{3}-\xi^{3}\right)^{1 / 3}, \xi\right)}{\left(\varsigma^{3}-\xi^{3}\right)^{2 / 3}} f\left(\eta,\left(\varsigma^{3}-\xi^{3}\right)^{1 / 3}\right) f(\eta, \xi) d \xi+S_{c}(\varsigma)
\end{gathered}
$$

where, the first term on the right-hand side of Eq. (44) represents breakup sink term of bubbles with diameter $\varsigma$ per unit time, the second term on the right-hand side of Eq. (44) represents breakup source term of bubbles with diameter $\varsigma$ per unit time, the third term on the right-hand side of Eq. (44) represents coalescence sink term of bubbles with diameter $\varsigma$ per unit time, the fourth term on the right-hand side of Eq. (44) represents coalescence source term of bubbles with diameter $\varsigma$ per unit time, the fifth term on the right-hand side of Eq. (44) represents bubbles with diameter $\varsigma$ source term.

The initial condition for Eq. (44) is given

$$
f(\eta, \varsigma, 0)=0
$$

The breakage frequency $b(\varsigma)$ is given as [28]

$$
\begin{gathered}
b(\varsigma)=\frac{\kappa_{1} \varepsilon^{1 / 3}}{\varsigma^{2 / 3}} \exp \left(-\frac{\sigma \kappa_{2}}{\rho_{g} \varepsilon^{2 / 3} \varsigma^{5 / 3}}\right) \\
\varepsilon=C_{g} u_{b} g
\end{gathered}
$$

The daughter bubble size redistribution function $h_{b}(\xi, \varsigma)$ is given as [28]

$$
h_{b}(\xi, \varsigma)=\frac{4.8}{\xi} \exp \left(-4.5 \frac{(2 \varsigma-\xi)^{2}}{\xi^{2}}\right)
$$

The coalescence closure is given as [28]

$$
\begin{gathered}
c(\varsigma, \xi)=0.05 \frac{\pi}{4}(\varsigma+\xi)^{2}\left[\beta_{c}(\varepsilon \varsigma)^{2 / 3}+\beta_{c}(\varepsilon \xi)^{2 / 3}\right]^{1 / 2} \\
\exp \left(-\frac{\left(r_{c}^{3} \rho_{w} / 16 \sigma\right)^{1 / 2} \varepsilon^{1 / 3} \ln \left(h_{i} / h_{f}\right)}{r_{c}^{2 / 3}}\right) \\
r_{c}=\frac{1}{4}\left(\frac{1}{\varsigma}+\frac{1}{\xi}\right)^{-1}
\end{gathered}
$$

The value of parameter $\beta_{c}$ is taken as 2.48. The expression of source term $S_{c}(\varsigma)$ is 


$$
S_{c}(\varsigma)=C_{\rho} \sqrt{\left(p_{v}-p\right)} \chi(\varsigma)
$$

where

$$
\begin{gathered}
\chi(\varsigma)=\int_{0}^{\varsigma}\left(\psi\left(\varsigma, \varsigma_{c}\right) g_{c}\left(\varsigma_{c}\right)\right) d \varsigma_{c} \\
C_{\rho}=\left(\frac{2}{3 \rho_{w}}\right)^{1 / 2}
\end{gathered}
$$

The redistribution function of bubble diameter $\varsigma$ is given as

$$
\psi\left(\varsigma_{c}, \varsigma\right)=\frac{c_{1}}{\varsigma_{c}} \exp \left(-\frac{c_{2}\left(\varsigma-\varsigma_{c}\right)^{2}}{\varsigma_{\max }^{2}}\right)
$$

The diameter distribution density function of the gas nucleus is given by experimental data. [29]

$$
g_{c}\left(\varsigma_{c}\right) \approx \frac{(\alpha+1)}{\varsigma_{c \max }} N_{i}\left(\frac{\varsigma_{c}}{\varsigma_{c \max }}\right)^{\alpha}
$$

where, the value of parameter $\alpha$ is taken as $-10 / 3$.

\section{Calculation of dynamic characteristics of the SGTB}

\subsection{Perturbation generalized Reynolds equations of cavitation water film}

As shown in Fig.4, the origin of Cartesian coordinate is located at the center of the stationary ring. The possible independent motions of the thrust disk are axial movement along $\mathrm{z}$ axis and angular movements around $\mathrm{x}$ and $\mathrm{y}$ axes. The perturbed displacements and velocities of the thrust disk with respect to the steady equilibrium position are defined as $\left(\Delta z, \Delta \varphi_{x}, \Delta \varphi_{y}\right)$ and $\left(\Delta \dot{z}, \Delta \dot{\varphi}_{x}, \Delta \dot{\varphi}_{y}\right)$. The assume initial position of the thrust disk is $\left(h_{0}, \varphi_{x 0}, \varphi_{y 0}\right)$, the film thickness in quasi-equilibrium can be expressed as

$$
h=h_{0}+\Delta z+r \cos \theta \Delta \varphi_{y}-r \sin \theta \Delta \varphi_{x}
$$

The perturbations will influence the pressure distribution in the water film through Reynolds equation. The high order terms are ignored, the transient pressure of water film can be expressed as

$$
p=p_{0}+p_{z} \Delta z+p_{\varphi_{x}} \Delta \varphi_{x}+p_{\varphi_{y}} \Delta \varphi_{y}+p_{\dot{z}} \Delta \dot{z}+p_{\dot{\varphi}_{x}} \Delta \dot{\varphi}_{x}+p_{\dot{\varphi}_{y}} \Delta \dot{\varphi}_{y}
$$


Substituting Eqs. (56) and (57) into Eq. (2), the zeroth and first-order perturbation generalized Reynolds equations in the $(\zeta-\eta)$ coordinates system are obtained for the SGTB respectively,

$$
\operatorname{Rey}()\left\{\begin{array}{c}
C_{w} p_{z} \\
C_{w} p_{\varphi_{x}} \\
C_{w} p_{\varphi_{y}} \\
C_{w} p_{\dot{z}} \\
C_{w} p_{\dot{\varphi}_{x}} \\
C_{w} p_{\dot{\varphi}_{y}}
\end{array}\right\}=\left\{\begin{array}{c}
q_{z} \\
q_{\varphi_{x}} \\
q_{\varphi_{y}} \\
q_{\dot{z}} \\
q_{\dot{\varphi}_{x}} \\
q_{\dot{\varphi}_{y}}
\end{array}\right\}
$$

where, the operator $\operatorname{Rey}()$ is defined as

$$
\begin{array}{r}
\frac{\partial}{\partial \eta}\left(\frac{h_{0}^{3}}{\bar{\mu}_{w} \zeta}\left(\frac{\zeta^{2} f^{\prime 2}(\zeta)}{k_{r}}+\frac{1}{k_{\theta}}\right) \frac{\partial}{\partial \eta}\right)+\frac{\partial}{\partial \zeta}\left(\frac{\zeta h_{0}^{3}}{\bar{\mu}_{w} k_{r}} \frac{\partial}{\partial \zeta}\right) \\
-\frac{\partial}{\partial \zeta}\left(\frac{h_{0}^{3}}{\bar{\mu}_{w} k_{r}} \zeta f^{\prime}(\zeta) \frac{\partial}{\partial \eta}\right)-\frac{\partial}{\partial \eta}\left(\frac{h_{0}^{3}}{\bar{\mu}_{w} k_{r}} \zeta f^{\prime}(\zeta) \frac{\partial}{\partial \zeta}\right)
\end{array}
$$

$q_{j}\left(j=z, \varphi_{x}, \varphi_{y}, \dot{z}, \dot{\varphi}_{x}, \dot{\varphi_{y}}\right)$ can be found in Appendix A.

The stiffness and damping coefficients in the $(\zeta-\eta)$ coordinates system can be found by the following integrals

$$
\begin{aligned}
& {\left[\begin{array}{ccc}
K_{z z} & K_{z \varphi_{x}} & K_{z \varphi_{y}} \\
K_{\varphi_{x} z} & K_{\varphi_{x} \varphi_{x}} & K_{\varphi_{x} \varphi_{y}} \\
K_{\varphi_{y} z} & K_{\varphi_{y} \varphi_{x}} & K_{\varphi_{y} \varphi_{y}}
\end{array}\right]=\int_{0}^{2 \pi} \int_{r_{\text {in }}}^{r_{\text {out }}}\left[\begin{array}{c}
-1 \\
G_{1}(\eta, \zeta) \\
-G_{2}(\eta, \zeta)
\end{array}\right]\left\{p_{z} p_{\varphi_{x}} p_{\varphi_{y}}\right\} \zeta d \zeta d \eta} \\
& {\left[\begin{array}{ccc}
C_{z z} & C_{z \varphi_{x}} & C_{z \varphi_{y}} \\
C_{\varphi_{x} z} & C_{\varphi_{x} \varphi_{x}} & C_{\varphi_{x} \varphi_{y}} \\
C_{\varphi_{y} z} & C_{\varphi_{y} \varphi_{x}} & C_{\varphi_{y} \varphi_{y}}
\end{array}\right]=\int_{0}^{2 \pi} \int_{r_{\text {in }}}^{r_{\text {out }}}\left[\begin{array}{c}
-1 \\
G_{1}(\eta, \zeta) \\
-G_{2}(\eta, \zeta)
\end{array}\right]\left\{p_{\dot{z}} p_{\dot{\varphi}_{x}} p_{\dot{\varphi}_{y}}\right\} \zeta d \zeta d \eta}
\end{aligned}
$$

where

$$
\begin{aligned}
& G_{1}(\eta, \zeta)=\zeta \sin (\eta+f(\zeta)) \\
& G_{2}(\eta, \zeta)=\zeta \cos (\eta+f(\zeta))
\end{aligned}
$$



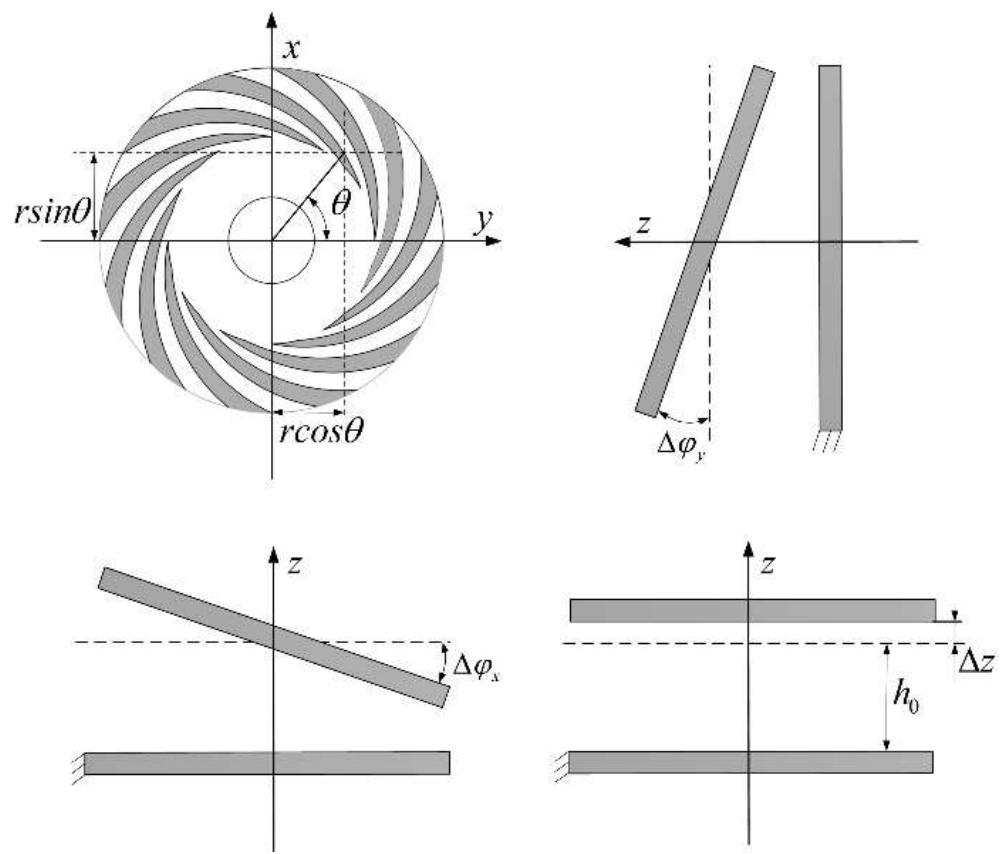

Fig. 4 Motion of a SGTB

\section{Numerical calculation method of theoretical model}

The generalized Reynolds equation (2), energy equation (21), and PBE (44) are the main three governing equations for the bearing. The pressure field, temperature field, and bubble diameter distribution are obtained by solving these three equations respectively, and pressure field; temperature field and bubble diameter distribution are coupled to each other, and an iterative algorithm needs to be employed to solve these distributions. Eq. (2) and Eq. (21) are discretized using finite difference, the pressure field is obtained by solving discretization equations of Eq. (2) with the SOR iterative method, the temperature field is obtained by solving discretization equations of Eq. (21) with the stepping method.

\subsection{Calculation of parameter $h_{c}^{*}$}

The shear stress parameter $h_{c}^{*}$ of the Couette flow is included in turbulent velocity distribution Eq. (2), The governing equation for parameter $h_{c}^{*}$ is written as [24]

$$
\left(h_{c}^{*}\right)^{2} \int_{0}^{1} \frac{d z^{*}}{f_{c}\left(z^{*}, h_{c}^{*}\right)}-R_{c}^{*} h^{*}=0
$$

Eq. (62) is a nonlinear equation with respect to $h_{c}^{*}$, the parameter $h_{c}^{*}$ can be solved using the Newton iteration method. The Newton iteration formula is expressed as $h_{c}^{*}$ 


$$
\left(h_{c}^{*}\right)^{(k+1)}=\left(h_{c}^{*}\right)^{(k)}-\frac{F\left(h_{c}^{*(k)}\right)}{F^{\prime}\left(h_{c}^{*(k)}\right)}
$$

where

$$
F\left(h_{c}^{*}\right)=\left(h_{c}^{*}\right)^{2} \int_{0}^{1} \frac{d z^{*}}{f_{c}\left(z^{*}, h_{c}^{*}\right)}-R_{c}^{*} h^{*}
$$

\subsection{Numerical solution of bubble PBE}

A discrete interval method [30] is employed to solve the bubble population balance Eq. (44). The procedure of the discrete interval method is as follows:

(1) Assume that the entire bubble diameter range is divided into $\mathrm{M}$ smaller intervals. The bubble diameter is approximated uniform distribution at kth representative diameter range $\left(\varsigma_{k}, \varsigma_{k+1}\right)$, then the $f(\eta, \varsigma)$ can be expressed by the bubble number density $n_{k}(\eta)$ at kth representative diameter range $\left(\zeta_{k}, \varsigma_{k+1}\right)$. Thus,

$$
f(\eta, \varsigma) \approx n_{k}(\eta) \delta\left(\varsigma-x_{k}\right)
$$

where

$$
n_{k}(\eta)=\int_{\zeta_{k}}^{\zeta_{k+1}} f(\eta, \varsigma) d \varsigma
$$

(2) The integrating the continuous Eq. (44) over a smaller range $\left(\varsigma_{k}, \varsigma_{k+1}\right)$. Thus,

$$
\begin{aligned}
& \frac{\partial}{\partial t} \int_{\zeta_{k}}^{\zeta_{k+1}} f(\eta, \varsigma) d \varsigma+u_{b} \frac{\partial}{\zeta \partial \eta}\left(\int_{\zeta_{k}}^{\zeta_{k+1}} f(\eta, \varsigma) d \varsigma\right)=-\int_{\zeta_{k}}^{\zeta_{k+1}} b(\eta, \varsigma) f(\eta, \varsigma) d \varsigma \\
& +\int_{\varsigma_{k}}^{\varsigma_{k+1}} d \varsigma \int_{\varsigma}^{\zeta_{\max }} h_{b}(\xi, \varsigma) b(\eta, \xi) f(\eta, \xi) d \xi \\
& -\int_{\varsigma_{k}}^{\varsigma_{k+1}} f(\eta, \varsigma) d \varsigma \int_{0}^{\zeta_{\max }} c(\varsigma, \xi) f(\eta, \xi) d \xi \\
& +\frac{1}{2} \int_{\varsigma_{k}}^{\varsigma_{k+1}} \varsigma^{2} d \varsigma \int_{0}^{\varsigma} \frac{c\left(\left(\varsigma^{3}-\xi^{3}\right)^{1 / 3}, \xi\right)}{\left(\varsigma^{3}-\xi^{3}\right)^{2 / 3}} f\left(\eta,\left(\varsigma^{3}-\xi^{3}\right)^{1 / 3}\right) f(\eta, \xi) d \xi \\
& +C_{\rho} \sqrt{\left(p_{v}-p\right)} \int_{\varsigma_{k}}^{\varsigma_{k+1}} \chi(\varsigma) d \varsigma
\end{aligned}
$$

(3) Since coalescence or breakage of bubbles of these diameters results in the formation of new bubbles whose diameters do not match with any of the node diameters, the new bubble diameter will be assigned to the adjoining node by introducing weight factor $\gamma$, the weight factor $\gamma$ can be determined by the total mass conservation of the bubble and the number of bubbles conservation. The expression is written as

$$
\begin{gathered}
\gamma_{k} x_{k}^{3}+\gamma_{k+1} x_{k+1}^{3}=\varsigma^{3} \\
\gamma_{k}+\gamma_{k+1}=1
\end{gathered}
$$

Substituting Eq. (65) into Eq. (67), and the integral term at the right end of Eq. (67) is reconstructed using the mean value theorem on breakage frequency and 
coalescence frequency, so Eq. (67) can be closed, a closed set of equations with regard to $n_{k}(\eta)$ can be written as

$$
\begin{gathered}
\frac{\partial n_{k}(\eta)}{\partial t}+u_{b} \frac{\partial}{\zeta \partial \eta}\left(n_{k}(\eta)\right)=-b\left(\eta, x_{k}\right) n_{k}(\eta) \\
+\sum_{i=k}^{M} \alpha_{k, i} b\left(\eta, x_{i}\right) n_{i}(\eta)-n_{k}(\eta) \sum_{i=1}^{M} c\left(\eta, x_{k}, x_{i}\right) n_{i}(\eta) \\
+\sum_{x_{k-1} \leq\left(x_{j}^{3}+x_{i}^{3}\right)^{1 / 3} \leq x_{k+1}}^{j \geq i}\left(1-\frac{1}{2} \delta_{j, i}\right) \gamma_{j, i}^{k} c\left(\eta, x_{j}, x_{i}\right) n_{j}(\eta) n_{i}(\eta) \\
+C_{\rho} \sqrt{\left(p_{v}-p\right)} \int_{\varsigma_{k}}^{\varsigma_{k+1}} \chi(\varsigma) d \varsigma
\end{gathered}
$$

where

$$
\begin{aligned}
& \gamma_{j, i}^{k}=\left\{\begin{array}{cc}
\frac{x_{k+1}^{3}-\left(x_{j}^{3}+x_{i}^{3}\right)}{\left(x_{k+1}^{3}-x_{k}^{3}\right)} & x_{k}^{3} \leq\left(x_{j}^{3}+x_{i}^{3}\right) \leq x_{k+1}^{3} \\
\frac{\left(x_{j}^{3}+x_{i}^{3}\right)-x_{k}^{3}}{\left(x_{k+1}^{3}-x_{k}^{3}\right)} & x_{k-1}^{3} \leq\left(x_{j}^{3}+x_{i}^{3}\right) \leq x_{k}^{3} \\
0 & \text { else }
\end{array}\right. \\
& k=1,2, \ldots \ldots M-2, \varsigma^{3}=\varsigma_{j}^{3}+\varsigma_{k}^{3} \\
& \gamma_{j, i}^{M-1}=\left\{\begin{array}{cc}
\frac{\left(x_{j}^{3}+x_{i}^{3}\right)}{x_{M-1}^{3}} & x_{k_{-} 1}^{3} \leq\left(x_{j}^{3}+x_{i}^{3}\right) \leq \varsigma_{M}^{3} \\
\frac{\left(x_{j}^{3}+x_{i}^{3}\right)-x_{M-2}^{3}}{\left(x_{M-1}^{3}-x_{M-2}^{3}\right)} & x_{M-2}^{3} \leq\left(x_{j}^{3}+x_{i}^{3}\right) \leq x_{M-1}^{3} \\
0 & \text { else }
\end{array}\right. \\
& \delta_{j, i}= \begin{cases}1 & j=i \\
0 & j \neq i\end{cases}
\end{aligned}
$$

Coefficient $\alpha_{k, i}$ is given as

$$
\alpha_{k, i}=\int_{x_{k-1}}^{x_{k}} \frac{\left(\varsigma^{3}-x_{k-1}^{3}\right)}{\left(x_{k}^{3}-x_{k-1}^{3}\right)} h_{b}\left(x_{i}, \varsigma\right) d \varsigma+\int_{x_{k}}^{x_{k+1}} \frac{\left(x_{k+1}^{3}-\varsigma^{3}\right)}{\left(x_{k+1}^{3}-x_{k}^{3}\right)} h_{b}\left(x_{i}, \varsigma\right) d \varsigma
$$

Eq. (69) is a set of first-order differential equations. Suppose that at a fixed time t, a set of nonlinear equations with regard to $n_{k, l}^{t}$ can be obtained by discretizing the second term on the left side of Eq. (69). The discrete equation at node $l$ is written as follows

$$
\tilde{Q}\left(n_{k, l}^{t}\right)=0
$$

where, the operator $\tilde{Q}\left(n_{k, l}\right)$ are defined as 


$$
\begin{gathered}
\tilde{Q}\left(n_{k, l}^{t}\right)=\frac{u_{b}}{\zeta_{m}} \frac{n_{k, l}^{t}-n_{k, l-1}^{t}}{\Delta \eta}+b\left(\eta_{l}, x_{k}\right) n_{k, l}^{t} \\
-\sum_{i=k}^{M} \alpha_{k, i} b\left(\eta_{l}, x_{i}\right) n_{i, l}^{t}+n_{k, l}^{t} \sum_{i=1}^{M} c\left(\eta_{l}, x_{k}, x_{i}\right) n_{i, l}^{t} \\
-\sum_{\substack{x_{k-1} \leq x_{j}+x_{i} \leq x_{k+1} \\
j \geq i}}\left(1-\frac{1}{2} \delta_{j, i}\right) \gamma_{j, i}^{k} c\left(\eta_{l}, x_{j}, x_{i}\right) n_{j, l}^{t} n_{i, l}^{t}-C_{\rho} \sqrt{p_{v}-p} \int_{\zeta_{k}}^{\zeta_{k+1}} \chi(\varsigma) d \varsigma
\end{gathered}
$$

Eq. (74) can be solved using the Newton-SOR iterative method. In addition, since $h_{b}\left(x_{k}, \varsigma\right)$ is a given function, the coefficient $\alpha_{k, i}$ can be calculated using the Gaussian integration method, and the value of the coefficient $\alpha_{k, i}$ is stored in a matrix, it will be called directly in the iterative calculation process, and the calculation time can be greatly saved by using the iterative process.

The term of the time derivative in the transient equation (69) is specially processed with finite difference, and a weighted averaging method is adopted for the timedifferencing. It can be written as [31]

$$
\frac{n_{k, l}^{t+1}-n_{k, l}^{t}}{\Delta t}+\left(\left(1-\omega_{t}\right) \tilde{Q}\left(n_{k, l}^{t}\right)+\omega \tilde{Q}\left(n_{k, l}^{t+1}\right)\right)=0
$$

where, $n_{k, l}^{t+1}=n_{k, l}(t+\Delta t)$, weight factor $0 \leq \omega_{t} \leq 1$.

A set of nonlinear equations with regard to $n_{k, l}^{t+1}$ can be rewritten as

$$
\begin{gathered}
F_{q}\left(n_{k, l}^{t+1}\right)=n_{k, l}^{t+1}+\Delta t \omega_{t} \tilde{Q}\left(n_{k, l}^{t+1}\right)+\Delta t\left(1-\omega_{t}\right) \tilde{Q}\left(n_{k, l}^{t}\right)-n_{k, l}^{t}=0 \\
\left(q=1, \ldots N_{q}\right)
\end{gathered}
$$

The nonlinear equation (77) is also solved using the Newton-SOR iterative method. And downhill condition is adopted in numerical iterative procedure to improve convergence. The downhill condition is expressed as

$$
\left\|F_{q}\left(n_{k, l}^{t+1(m+1)}\right)\right\| \leq\left\|F_{q}\left(n_{k, l}^{t+1(m)}\right)\right\|
$$

where $m$ is iteration number. The overall algorithm flow diagram is given by Fig. 5. 


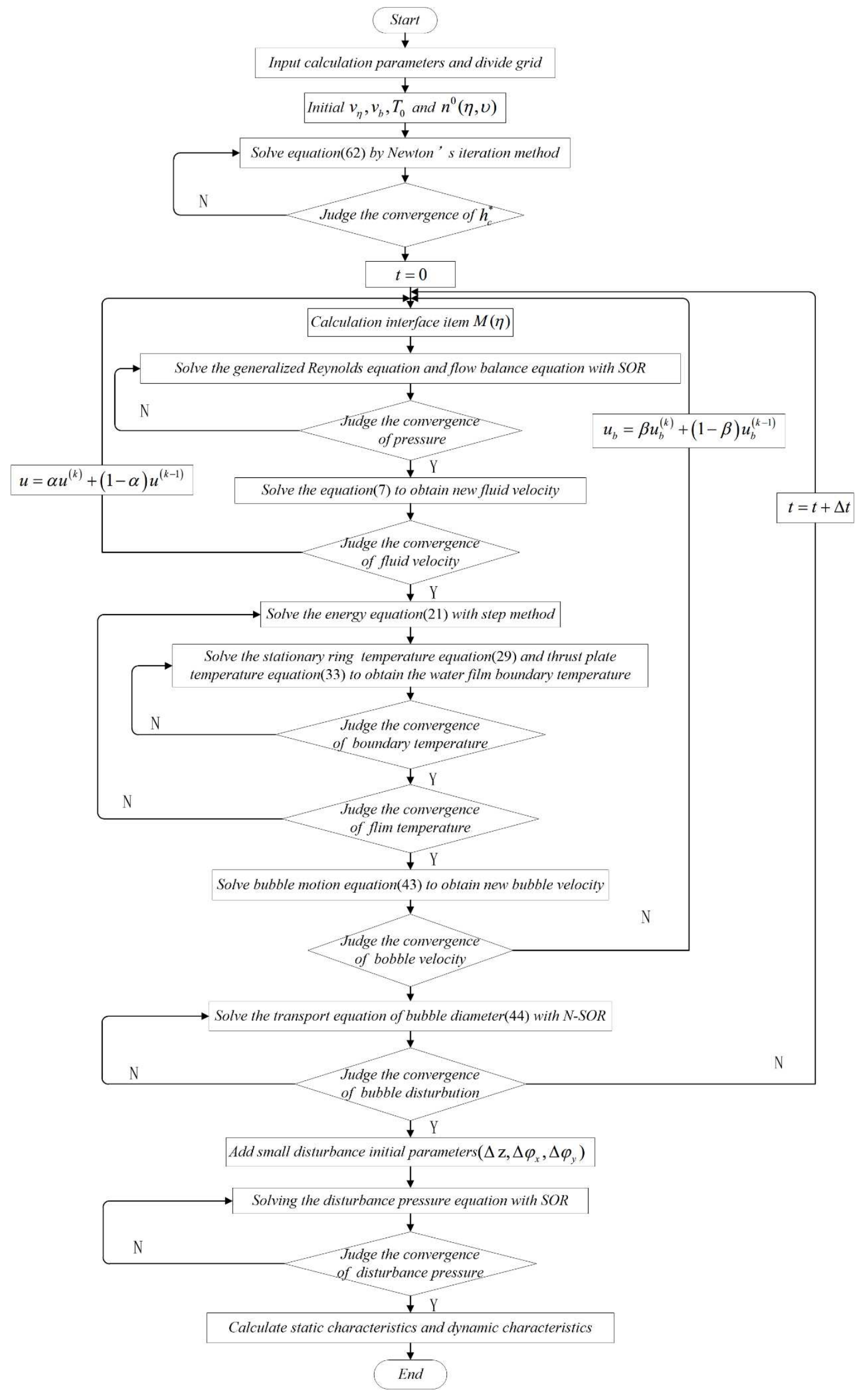


Fig. 5 Flow diagram of overall algorithm

\section{Results and Discussion}

\subsection{Experimental verification of theoretical models}

Table 1 list the related parameters of the water-lubricated SGTB for the numerical calculation. In order to verify this theoretical model, the simulating results of bubble distribution and axial stiffness coefficient are compared with experimental ones from our previous test study in Refs.[22,23].

Table 1 The parameters of the water-lubricated SGTB

\begin{tabular}{cc}
\hline Item & Value \\
\hline Inner radius $r_{\text {in }}(\mathrm{mm})$ & 7.5 \\
Outer radius $r_{\text {out }}(\mathrm{mm})$ & 20 \\
Base circle radius $r_{b}(\mathrm{~mm})$ & 12 \\
Water viscosity $\mu(P a . s)$ & $0.001\left(20^{\circ}\right)$ \\
Groove number $N$ & 12 \\
Groove depth $h_{g}(\mu m)$ & 40 \\
Spiral angles $\beta(\circ)$ & 20 \\
Pressure of supply water $p_{\text {in }}(\mathrm{MPa})$ & 0.1 \\
Specific heat at constant volume of water & 4200 \\
$c_{v}\left(\mathrm{~J} / \mathrm{kg} \cdot \mathrm{C}^{\circ}\right)$ & 1000 \\
Density of water $\rho_{w}\left(\mathrm{~kg} / \mathrm{m}^{3}\right)$ & 20 \\
Bearing capacity $W(\mathrm{~N})$ & 0.3 \\
Surface tension of bubble $\sigma(\mathrm{N} / \mathrm{m})$ & 0.5 \\
Groove-to-land ratio $\lambda_{b}$ & 0.6 \\
Groove-to-dam ratio $\lambda_{l}$ &
\end{tabular}

\section{1) Bubble distribution}

Fig. 6 shows the photographs of bubble distributions taken during the experiments under the rotational speeds of $15000 \mathrm{rpm}$ and $18000 \mathrm{rpm}$. 


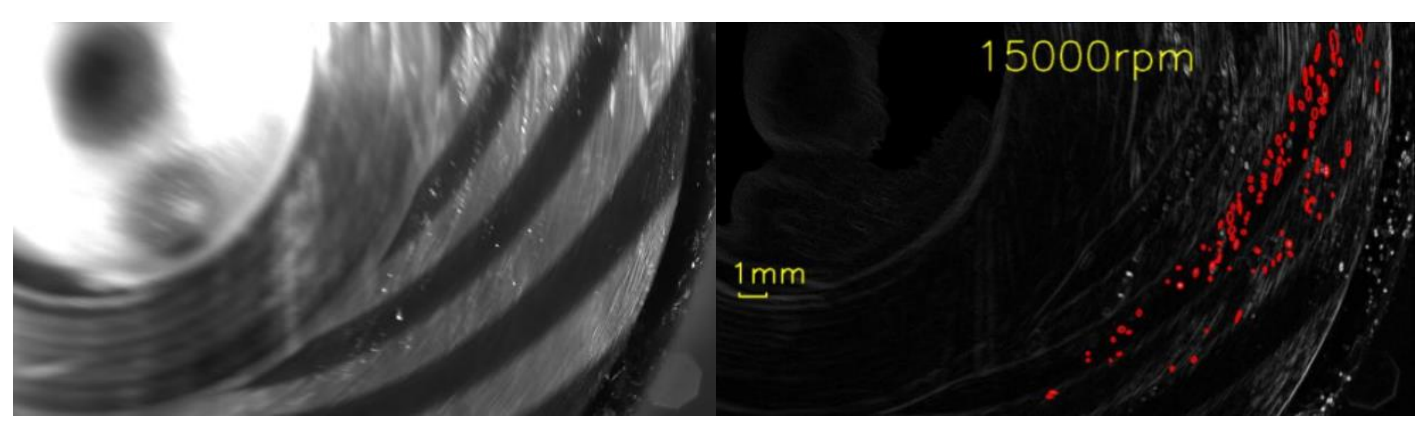

(a) 15000rpm

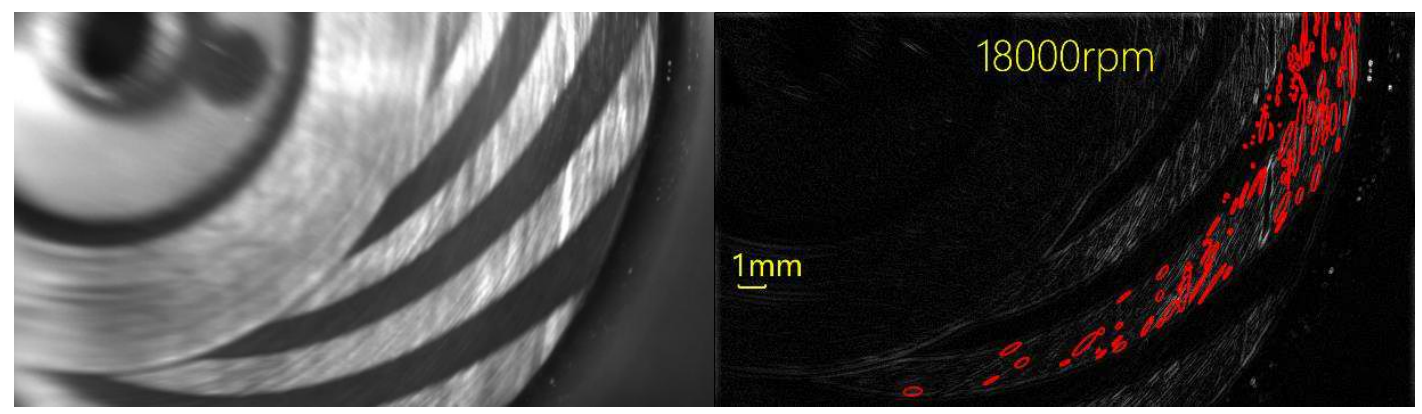

(b) 18000rpm

Fig.6 Photographs of bubble distributions under both rotational speeds: (a) $15000 \mathrm{rpm}$, (b) $18000 \mathrm{rpm}$

The bubble size distribution experimental data are counted from the photos. A comparison of the theoretical results of the bubble size distribution with the experimental statistics results is shown in Fig.7. It can be seen that, for a specified rotational speed of $15000 \mathrm{rpm}$, the results predicted by the model are in good agreement with the experimental statistics results over the entire bubble size range; while, for a specified rotational speed of $18000 \mathrm{rpm}$, the results predicted by the model are in good agreement with the experimental statistics results in the large size bubble range, but the deviation between the theoretical calculation and the experimental statistics results is large in the small size bubble range, and the experimental statistical values are all larger than the theoretical ones in the small size bubble range. This may be explained by the fact that the number of bubbles is excessively counted in the small-sized bubble range from the experimental photos, since there exists an error unavoidably when the edge contours of small size bubbles were identified from the photos. 


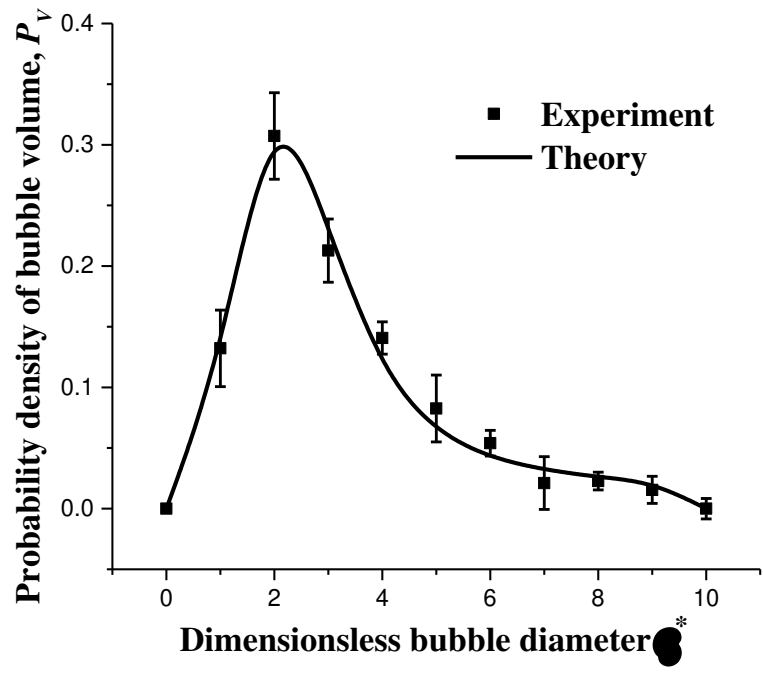

(a) 15000rpm

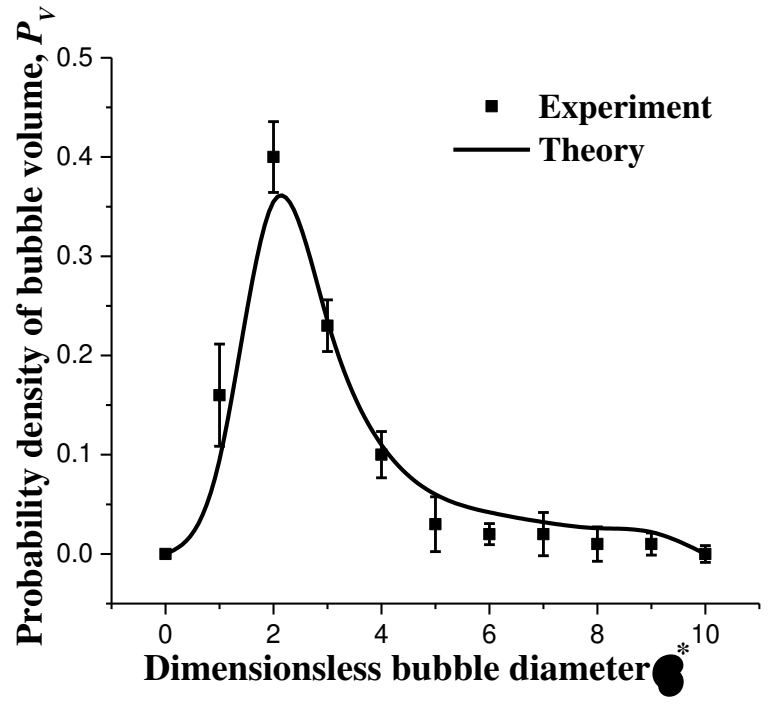

(b) 18000rpm

Fig. 7 Comparison of the theoretical probability density distributions with the experimental ones: (a)15000rpm, (b)18000rpm

$$
\left(\beta=20^{\circ}, h_{s}=15 \mu m, h_{g}=40 \mu m\right)
$$

\section{2) Axial stiffness coefficient}

A comparison of the predicting axial stiffness coefficients with the experimental ones is shown in Fig. 8. It can be seen that the predicting stiffness coefficients with axial load are generally in agreement with the experimental ones, but the experimental values are larger than the predicting ones in the entire range of applied load. This may be explained by the fact that the actual water flowrate of the bearing is less than the 
theoretical one, because the water is tended to be prevented from entering the clearance of inward-pumping SGTB due to the centrifugal effect.

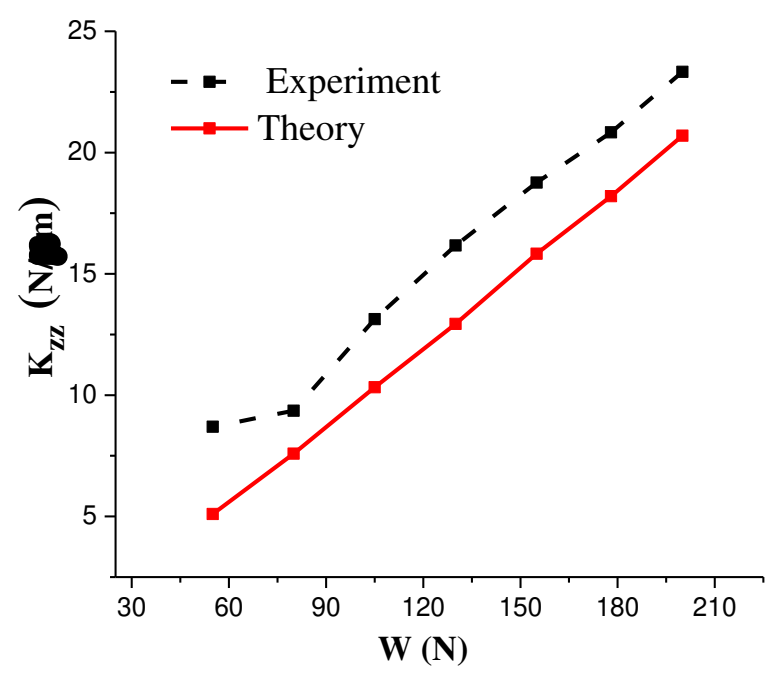

Fig. 8 Comparison of the theoretical stiffness coefficients with the experimental ones at 15000rpm

\subsection{Influence of cavitation effect on dynamic characteristics}

1) Stiffness coefficient

Fig. 9 shows the influence of cavitation effect on the stiffness coefficients of the SGTB with different spiral angles. The results show the direct stiffness coefficients $\left(K_{z z}^{*}, K_{\varphi_{x} \varphi_{x}}^{*}, K_{\varphi_{y} \varphi_{y}}^{*}\right)$ of the cavitating flow are larger than those with noncavitating flow when the spiral angle is less than 50 degrees. This may be explained by the fact that the bubbles cause the interfacial hydrodynamic effect for the the cavitating flow. In addition, the relative deviation between the cavitating flow model and the noncavitating flow model decreases with the increasing of spiral angle, so the interface hydrodynamic effect of the bubbles decreases with the spiral angle. It means that the influence of the cavitation effect on the water film stiffness is weakened with the increasing of spiral angle. The cross-coupled stiffness coefficients $\left(K_{\varphi_{x} \varphi_{y}}^{*}, K_{\varphi_{y} \varphi_{x}}^{*}\right)$ are 
of opposite sign, and $K_{\varphi_{x} \varphi_{y}}^{*}$ and $K_{\varphi_{y} \varphi_{x}}^{*}$ of cavitating flow with the angle generally coincide with those of non-cavitating flow. It shows that the influence of cavitation effect on cross-coupled stiffness coefficients $\left(K_{\varphi_{x} \varphi_{y}}^{*}, K_{\varphi_{y} \varphi_{x}}^{*}\right)$ are negligible. In addition, the cross-coupled stiffness $\left(K_{\varphi_{x} z}^{*}, K_{z \varphi_{x}}^{*}, K_{\varphi_{y} z}^{*}, K_{z \varphi_{y}}^{*}\right)$ with different spiral angles are approximately equal to zero for the cavitating flow or the non-cavitating flow.

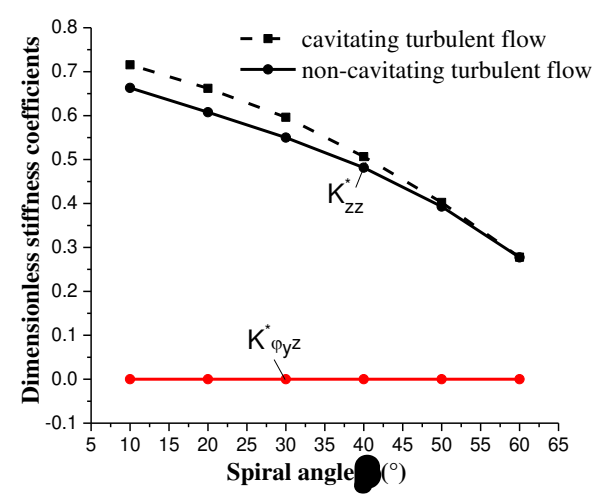

(a)

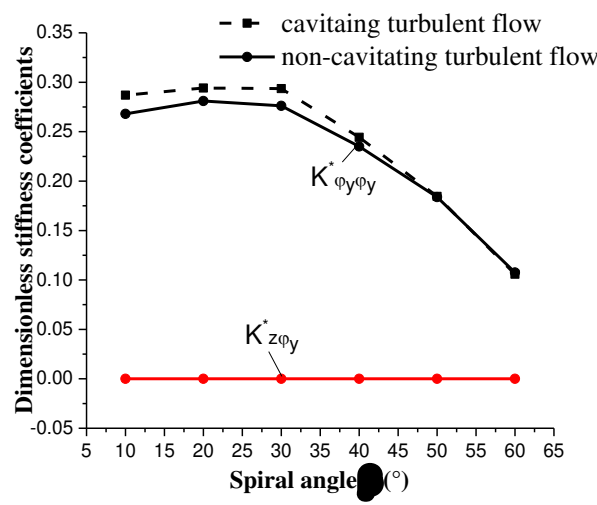

(c)

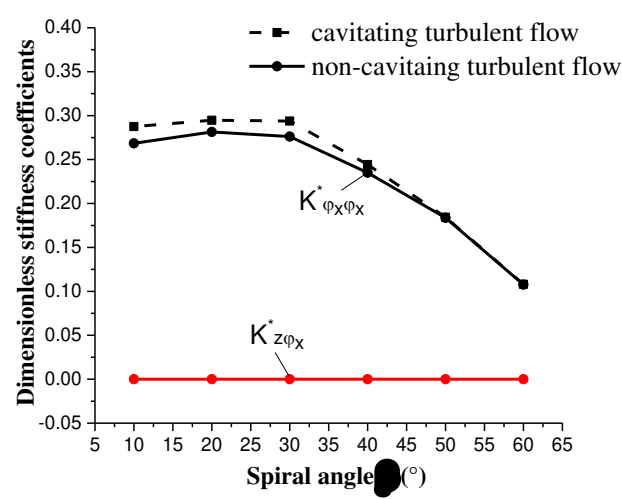

(b)

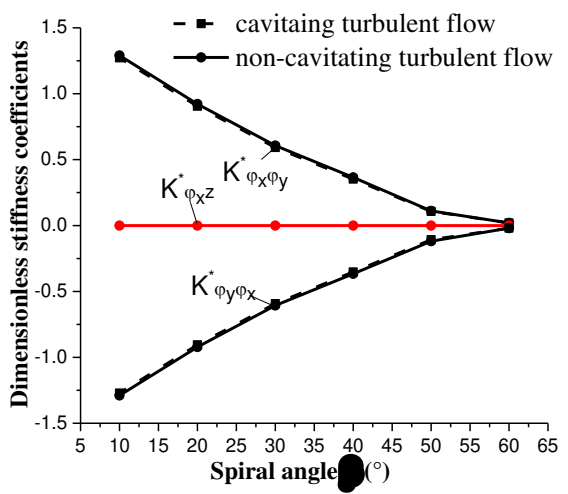

(d)

Fig. 9 Influence of cavitation effect on the stiffness coefficient for different spiral angles

$$
\left(\omega=18000 \mathrm{rpm}, h_{s}=15 \mu \mathrm{m}, h_{g}=40 \mu \mathrm{m}\right)
$$

Fig. 10 shows the influence of cavitation effect on the stiffness coefficient of the SGTB with different groove depth. The result also show the direct stiffness coefficients $\left(K_{z z}^{*}, K_{\varphi_{x} \varphi_{x}}^{*}, K_{\varphi_{y} \varphi_{y}}^{*}\right)$ of cavitating flow are larger than those of non-cavitating flow at a 
specific groove depth. This is also due to the fact that the bubbles cause the interfacial hydrodynamic effect for the cavitating flow. However, the differences of direct stiffness coefficients $\left(K_{z z}^{*}, K_{\varphi_{x} \varphi_{x}}^{*}, K_{\varphi_{y} \varphi_{y}}^{*}\right)$ between the cavitating flow and the non-cavitating flow increase first and then decrease with the groove depth. The step hydrodynamic effect becomes weak when the groove depth exceeds a given value, so the velocity difference between fluid and bubble reduces and the bubble density increases due to the weakening of hydrodynamic effect. The interface hydrodynamic effect is positively correlated with the velocity difference and the bubble density. Therefore, the interface hydrodynamic effect changes with the groove depth, meaning that the influence of cavitation effect on stiffness depends on the groove depth.

In addition, the variation of the cross-coupled stiffness coefficients $\left(K_{\varphi_{x} \varphi_{y}}^{*}, K_{\varphi_{y} \varphi_{x}}^{*}\right)$ with the groove depth is identical with that with the spiral angle. Similarly, it also shows that the influence of cavitation effect on cross-coupled angular stiffness coefficients $\left(K_{\varphi_{x} \varphi_{y}}^{*}, K_{\varphi_{y} \varphi_{x}}^{*}\right)$ are negligible for all the groove depths. The cross-coupled stiffness coefficients $\left(K_{\varphi_{x} z}^{*}, K_{z \varphi_{x}}^{*}, K_{\varphi_{y} z}^{*}, K_{z \varphi_{y}}^{*}\right)$ with different groove depths are approximately equal to zero for the cavitating flow or the non-cavitating flow.

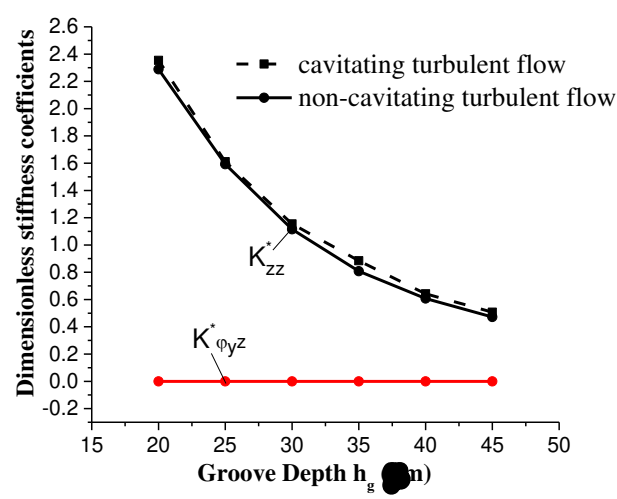

(a)

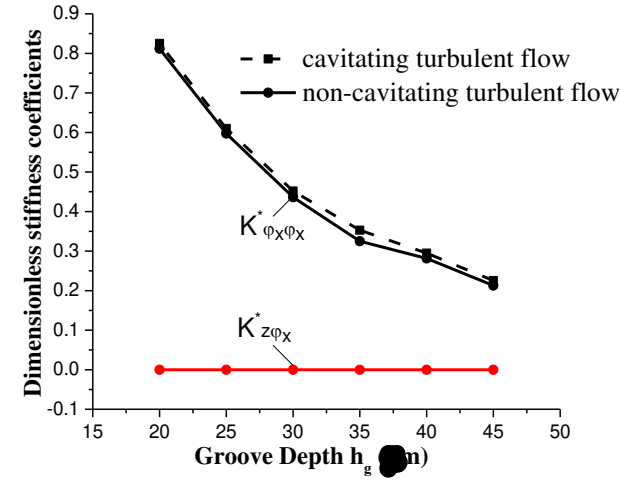

(b) 


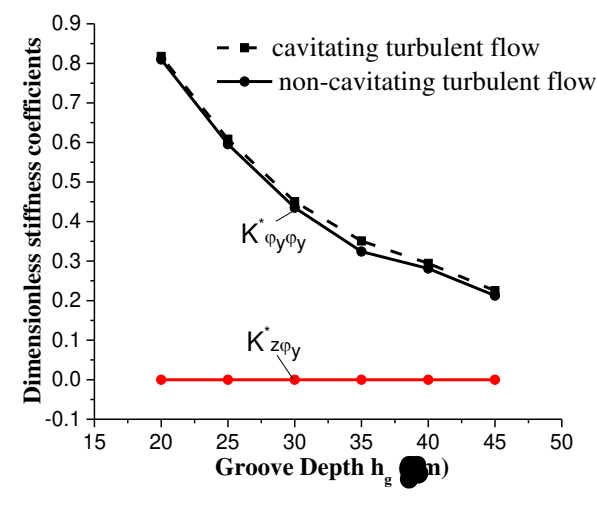

(c)

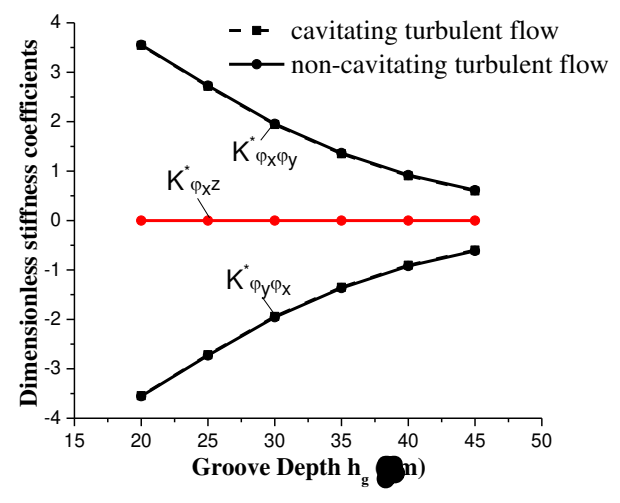

(d)

Fig. 10 Influence of cavitation effect on stiffness coefficients for different

groove depth

$$
\left(\omega=18000 \mathrm{rpm}, \beta=20^{\circ}, h_{s}=15 \mu \mathrm{m}\right)
$$

Fig. 11 shows the influence of cavitation effect on the stiffness coefficient of the SGTB with different rotating speed. It can be seen that the direct stiffness coefficients $\left(K_{z z}^{*}, K_{\varphi_{x} \varphi_{x}}^{*}, K_{\varphi_{y} \varphi_{y}}^{*}\right)$ with cavitating flow are larger than those with non-cavitating flow at a specific rotating speed. This is also due to the interfacial hydrodynamic effect caused by the bubbles. The cross-coupled stiffness coefficients $\left(K_{\varphi_{x} \varphi_{y}}^{*} K_{\varphi_{y} \varphi_{x}}^{*}\right)$ of cavitating flow are approximately equal to those of non-cavitating flow for different rotating speeds. The difference of direct stiffness coefficients $\left(K_{z z}^{*}, K_{\varphi_{x} \varphi_{x}}^{*}, K_{\varphi_{y} \varphi_{y}}^{*}\right)$ between the cavitating flow and the non-cavitating flow gradually increase as the rotating speed increases. This also can be explained by the fact that the hydrodynamic effect enhances as the rotational speed increases, and the velocity difference between the fluid and the bubble increases with this hydrodynamic effect. It means that the influence of the cavitation effect on stiffness is increased with the increase of rotating speed. The cross-coupled stiffness $\left(K_{z \varphi_{x}}^{*}, K_{\varphi_{x} z}^{*}, K_{z \varphi_{y}}^{*}, K_{\varphi_{y} z}^{*}\right)$ for different rotating speed are also approximately equal to zero for the cavitating flow or 
the non-cavitating flow.

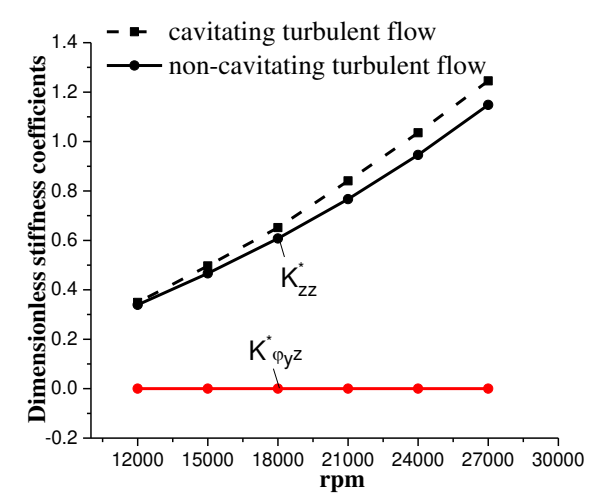

(a)

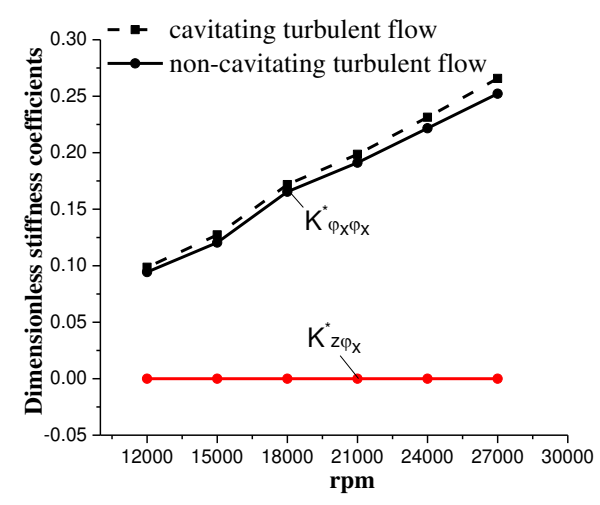

(c)

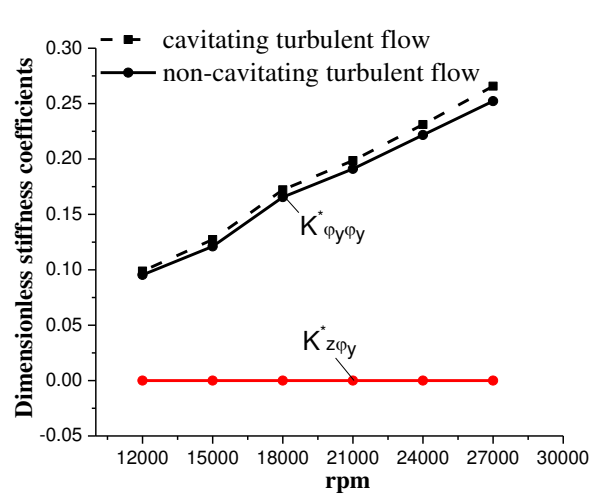

(b)

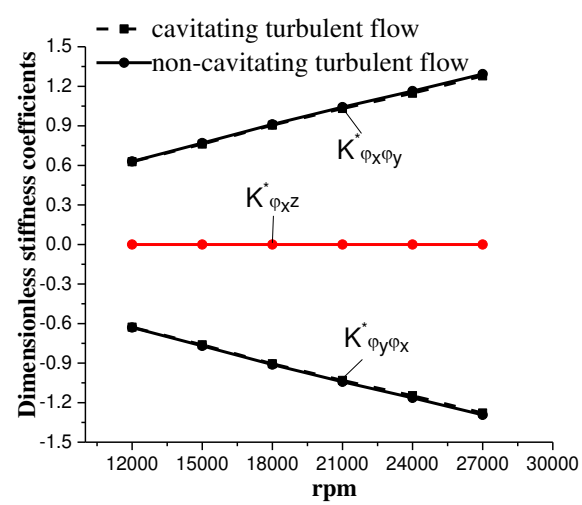

(d)

Fig. 11 Influence of cavitation effect on stiffness coefficients for different rotating speed

$$
\left(\beta=20^{\circ}, h_{s}=15 \mu m, h_{g}=40 \mu m\right)
$$

\section{2) Damping coefficient}

Fig. 12 shows the influence of cavitation effect on the water film damping coefficients for different spiral angles. It can be seen that the cavitation effect on damping coefficients is very weak. Because the damping coefficient is determined by the perturbed pressure, and the perturbed pressure with cavitating flow is only affected by the fraction $C_{g}$ of bubble volume, and the variation of bubble volume fraction is 
very small for the cavitating flow. In addition, the direct damping coefficients $\left(C_{z z}^{*}, C_{\varphi_{x} \varphi_{x}}^{*}, C_{\varphi_{y} \varphi_{y}}^{*}\right) \quad$ decreases with the increasing of the spiral angle, and the crosscoupled damping coefficients $\left(C_{z \varphi_{x}}^{*}, C_{\varphi_{x} z}^{*}, C_{z \varphi_{y}}^{*}, C_{\varphi_{y} z}^{*}, C_{\varphi_{x} \varphi_{y}}^{*}, C_{\varphi_{y} \varphi_{x}}^{*}\right)$ with the cavitating flow and the non-cavitating flow are also approximately equal to zero for different spiral angles.

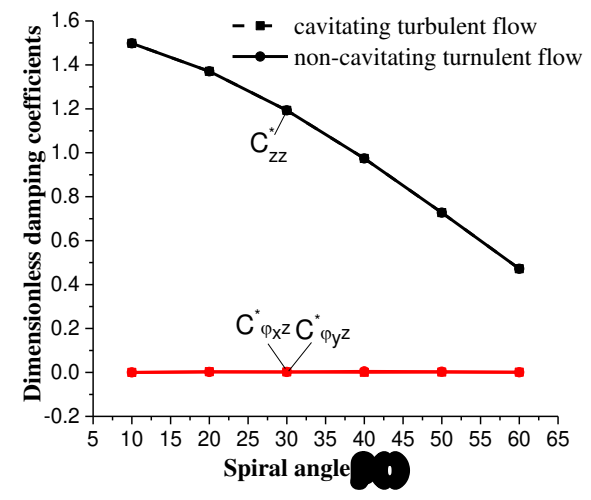

(a)

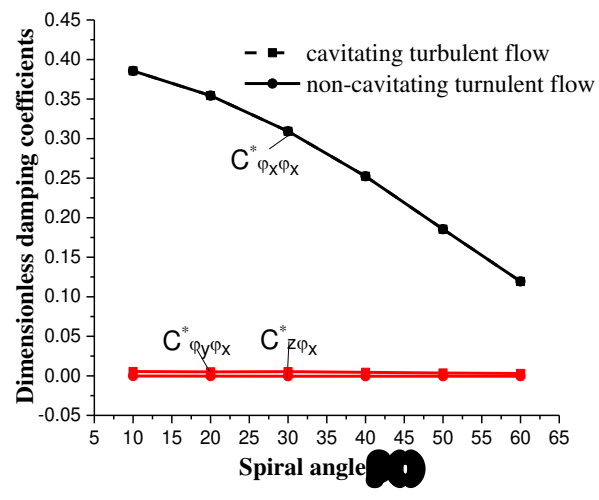

(b)

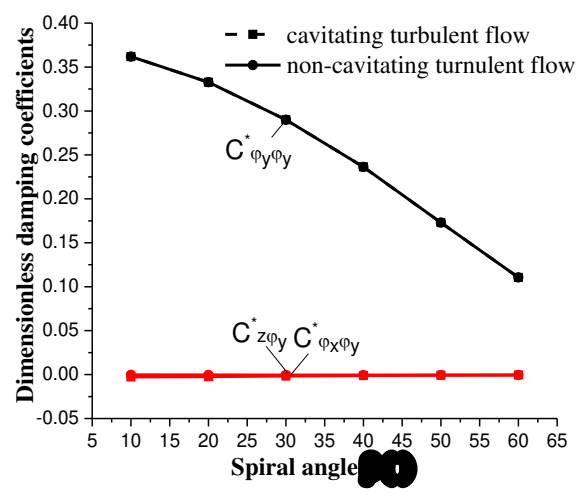

(c)

Fig. 12 Influence of cavitation effect on the damping coefficients for different spiral angles

$$
\left(\omega=18000 \mathrm{rpm}, h_{s}=15 \mu \mathrm{m}, h_{g}=40 \mu \mathrm{m}\right)
$$

Fig. 13 shows the influence of cavitation effect on the water film damping coefficients for different groove depth. It can be seen that the cavitation effect on damping coefficients is very weak for different groove depth. And the cross-coupled damping coefficients $\left(C_{z \varphi_{x}}^{*}, C_{\varphi_{x} z}^{*}, C_{z \varphi_{y}}^{*}, C_{\varphi_{y} z}^{*}, C_{\varphi_{x} \varphi_{y}}^{*}, C_{\varphi_{y} \varphi_{x}}^{*}\right)$ with the cavitating flow 
and the non-cavitating flow are also approximately equal to zero for different groove depth.

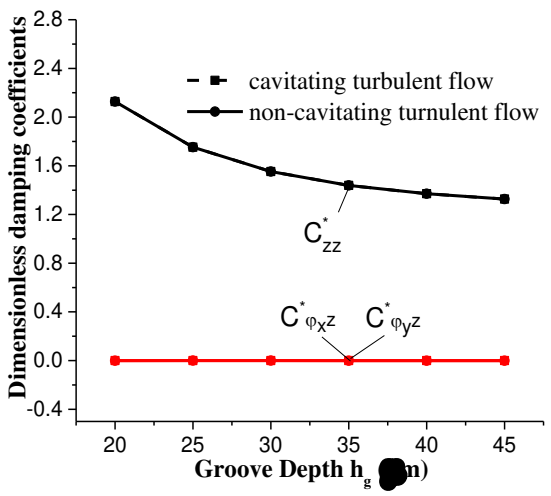

(a)

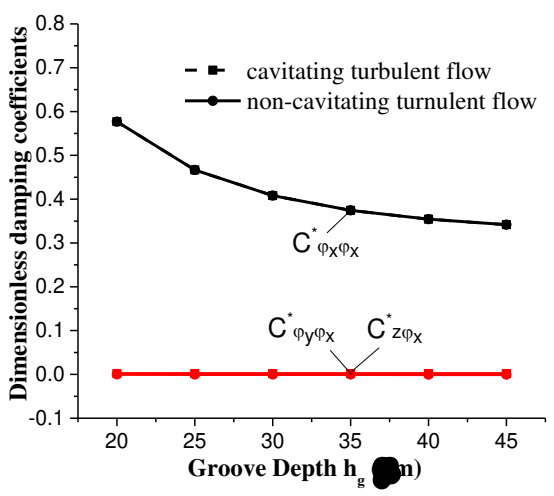

(b)

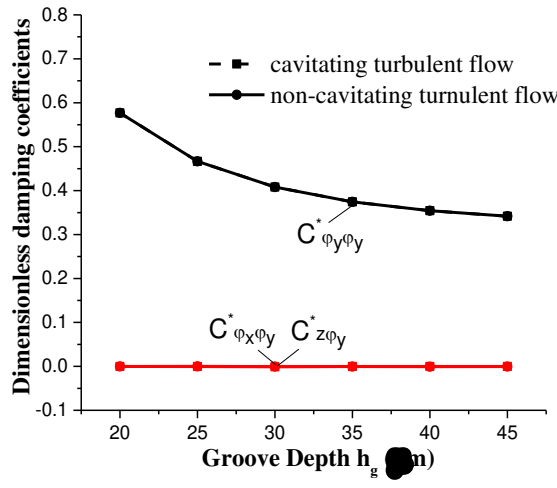

(c)

Fig. 13 Influence of cavitation effect on damping coefficients for different groove depth.

$$
\left(\omega=18000 \mathrm{rpm}, \beta=20^{\circ}, h_{s}=15 \mu \mathrm{m}\right)
$$

\subsection{Influence of turbulence effect on dynamic characteristics}

1) Stiffness coefficient

Fig.14 shows the influence of turbulence effect on the water film stiffness coefficients for different spiral angles. It can be seen that the direct stiffness coefficients $\left(K_{z z}^{*}, K_{\varphi_{x} \varphi_{x}}^{*}, K_{\varphi_{y} \varphi_{y}}^{*}\right)$ and the cross-coupled stiffness coefficients $\left(K_{\varphi_{x} \varphi_{y}}^{*}, K_{\varphi_{y} \varphi_{x}}^{*}\right)$ of turbulent cavitating flow are larger than those of the laminar cavitating flow at a specified spiral angle. This may be explained by the fact that additional Reynolds shear stress generates due to pulsating velocity in the turbulent 
flow, and equivalent viscosity of the turbulent water film increases, and the force of the turbulent water film needed to produce unit displacement increases, so the stiffness of the water film increases. In addition, it can be seen that the deviation of stiffness coefficient between turbulence model and laminar model gradually decreases with the increase of the spiral angle. This is due to the fact that the outward pumping hydrodynamic effect increases first and then decreases with the increase of the spiral angle. Therefore, when the spiral angle exceeds a certain value, the outward pumping hydrodynamic effect becomes weak and the additional Reynolds shear stress of the turbulent flow reduces. This indicates that the influence of turbulence effect on the water film stiffness coefficients is weakened when the spiral angle exceeds a certain value.

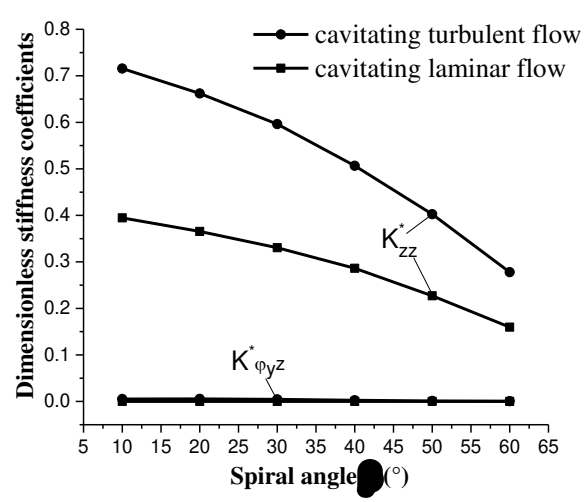

(a)

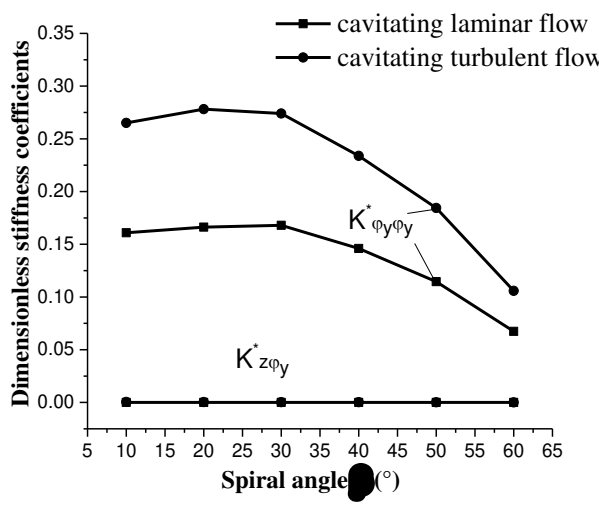

(c)

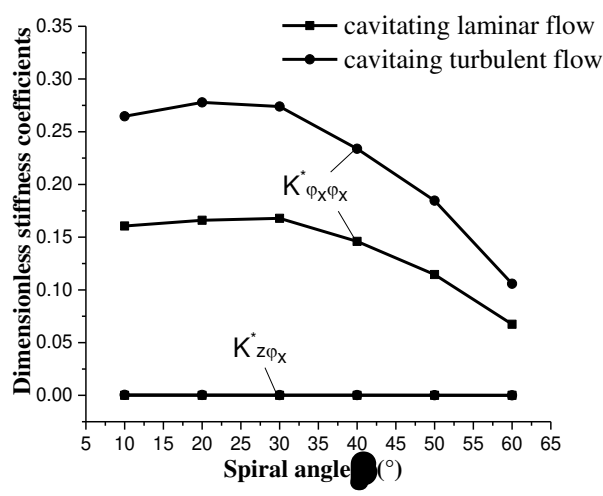

(b)

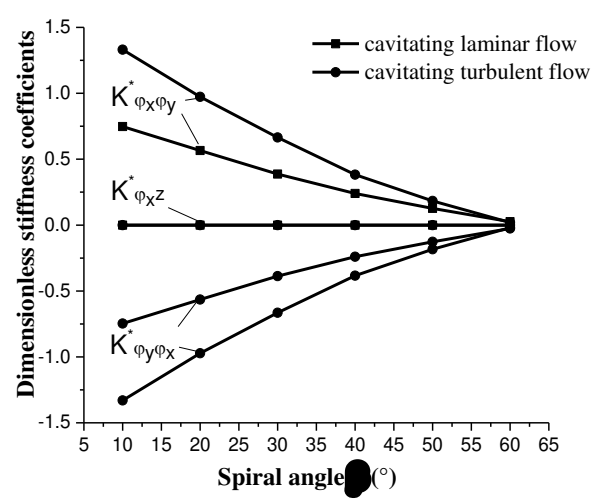

(d)

Fig. 14 Influence of turbulence effect on the water film stiffness coefficient for different spiral angles 


$$
\left(\omega=18000 \mathrm{rpm}, h_{s}=15 \mu \mathrm{m}, h_{g}=40 \mu \mathrm{m}\right)
$$

Fig. 15 shows the influence of turbulence effect on the water film stiffness coefficients for different groove depth. It can also be seen that the direct stiffness coefficients $\left(K_{z z}^{*}, K_{\varphi_{x} \varphi_{x}}^{*}, K_{\varphi_{y} \varphi_{y}}^{*}\right)$ and the cross-coupled stiffness coefficients of the turbulent cavitating flow are larger than those of the laminar cavitating flow at a specified groove depth. And the deviation of stiffness coefficients between turbulence model and laminar model also reduce with the increase of groove depth. Similarly, the step hydrodynamic effect becomes weak when the groove depth exceeds a given groove depth. This also show that the influence of turbulence effect on water film stiffness coefficients are also weakened when the groove depth exceeds a certain value.

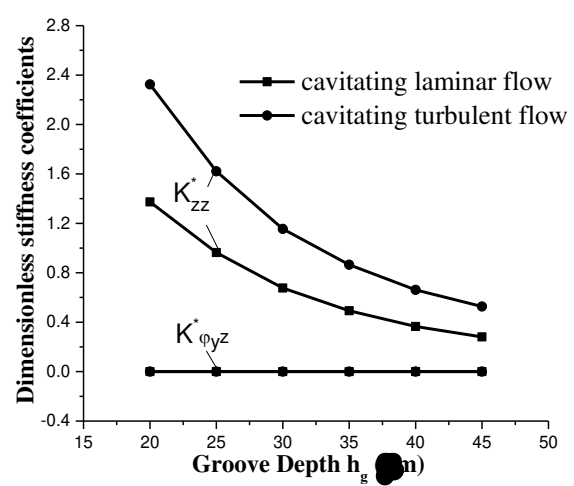

(a)

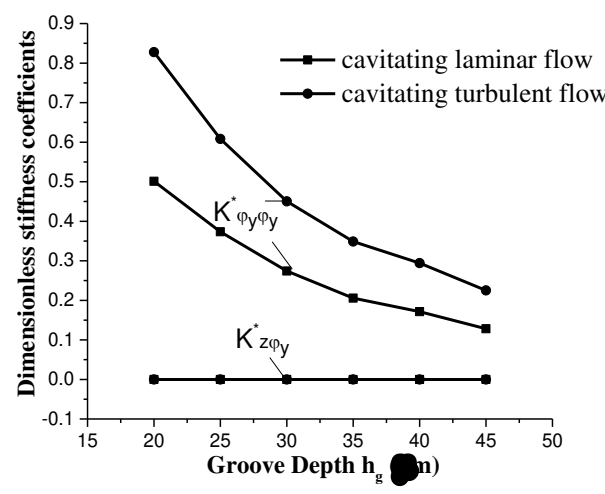

(c)

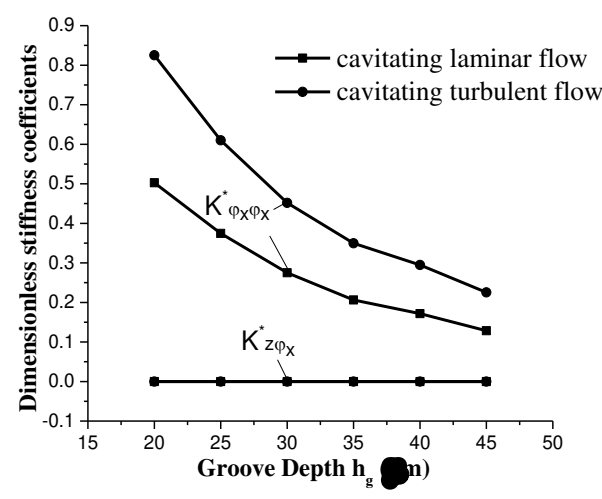

(b)

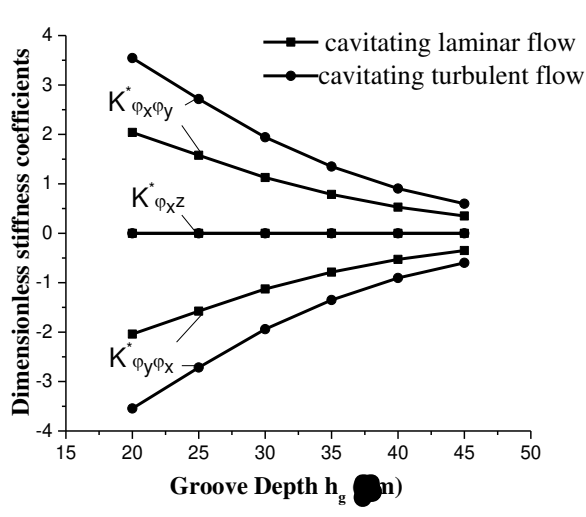

(d)

Fig. 15 Influence of turbulence effect on the stiffness coefficient for different groove depths

$$
\left(\omega=18000 \mathrm{rpm}, \beta=20^{\circ}, h_{s}=15 \mu \mathrm{m}\right)
$$

Fig. 16 shows the influence of turbulence effect on the water film stiffness 
coefficients for different rotating speed. It can also be seen that the direct stiffness coefficients and the cross-coupled stiffness coefficients of turbulent cavitating flow are larger than those of the laminar cavitating flow at a specified rotating speed. However, the deviation between turbulence model and laminar model is gradually increased with the increasing of rotating speed, the reason for this result is that the additional Reynolds shear stress of the turbulent flow increases with the rotational speed, leading to an enhancement of turbulence intensity and a larger deviation between turbulence model and laminar model.

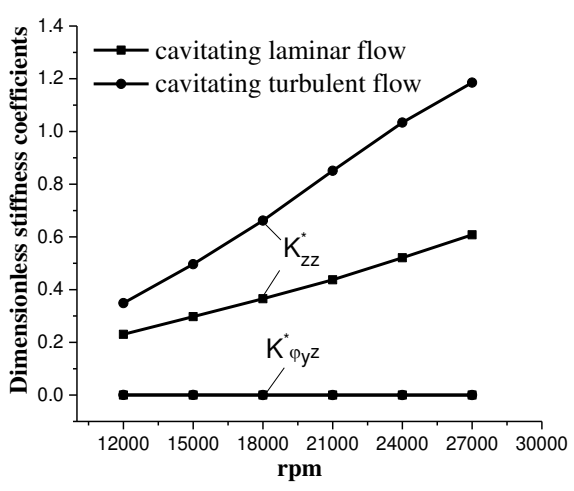

(a)

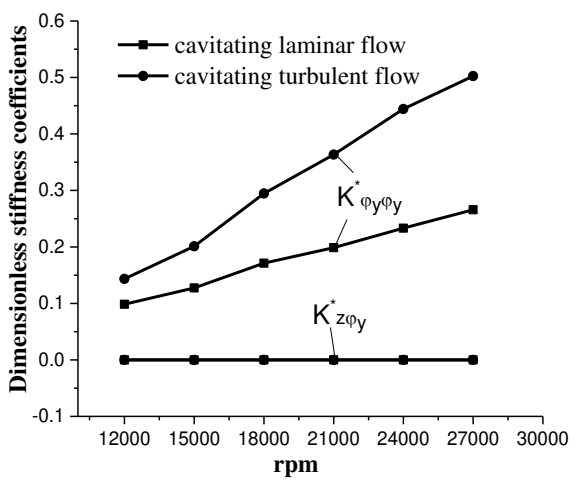

(c)

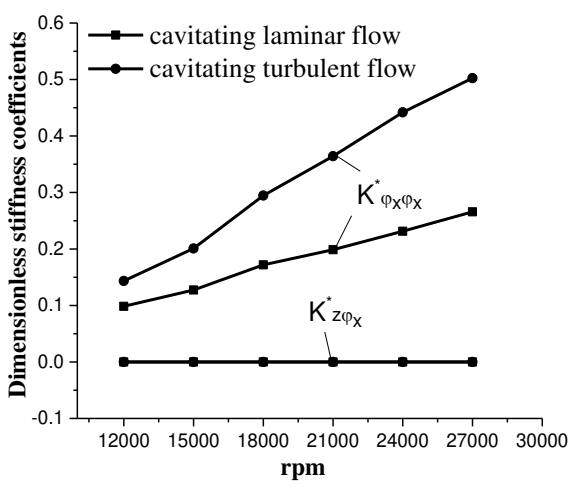

(b)

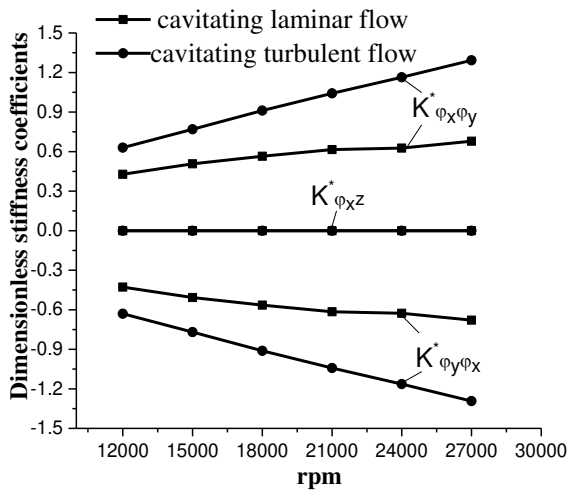

(d)

Fig. 16 Influence of turbulence effect on the stiffness coefficient for different rotating speed

$$
\left(\beta=20^{\circ}, h_{s}=15 \mu m, h_{g}=40 \mu m\right)
$$




\section{2) Damping coefficient}

Fig. 17 shows the influence of turbulence effect on the water film damping coefficients for different spiral angles. It can also be seen that the direct damping coefficients with turbulent cavitating flow are larger than those with the laminar cavitating flow at a specified spiral angle. The equivalent viscosity of water film is increased, and the force of the turbulent water film needed to produce unit velocity is increased, so the damping of the turbulent water film is increased. Similar to Fig. 14, the deviation of damping coefficients between turbulence model and laminar model is also gradually decreased with the increase of the spiral angle, correspondingly, the influence of the turbulence effect on the disturbance pressures s $p_{\dot{z}}, p_{\dot{\varphi}_{x}}, p_{\dot{\varphi}_{y}}$ is also weakened. This indicates that the influence of turbulence effect on damping coefficient of water film is weakened when the spiral angle exceeds a certain value.

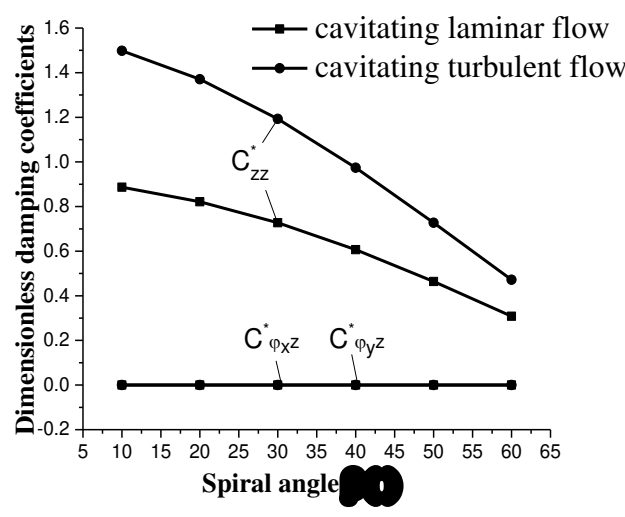

(a)

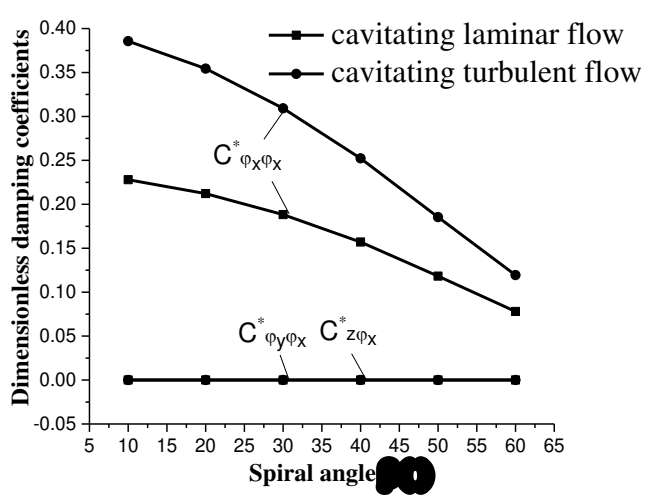

(b)

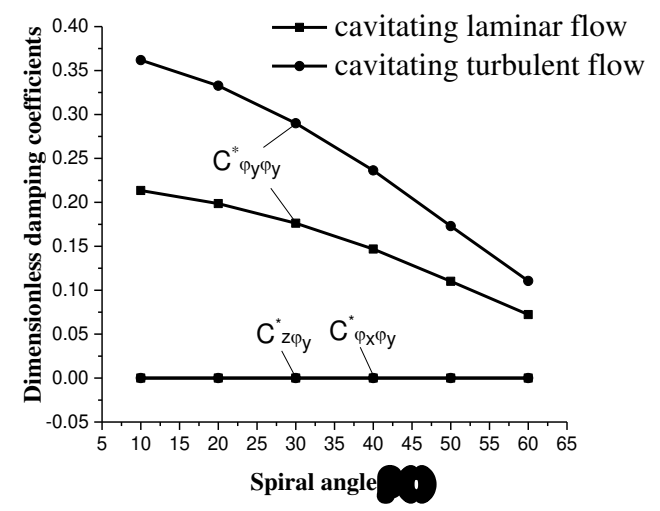

(c)

Fig. 17 Influence of turbulence effect on the damping coefficients for different 


\section{spiral angles.}

$$
\left(\omega=18000 \mathrm{rpm}, h_{s}=15 \mu \mathrm{m}, h_{g}=40 \mu \mathrm{m}\right)
$$

Fig.18 shows the influence of turbulence effect on the water film damping coefficients for different groove depth. It can also be seen that the direct damping coefficients with turbulent cavitating flow are larger than those with the laminar cavitating flow at a specified groove depth. However, the deviation of damping coefficient between turbulence model and laminar model decreases slowly with the increase of the groove depth. This indicates that the turbulence effect on damping coefficient is not sensitive to the groove depth.

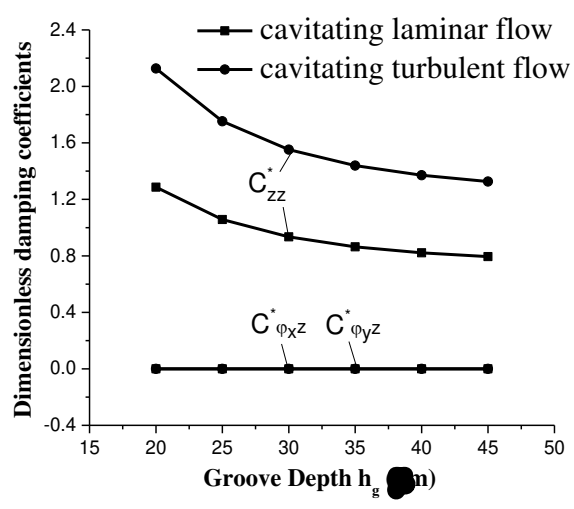

(a)

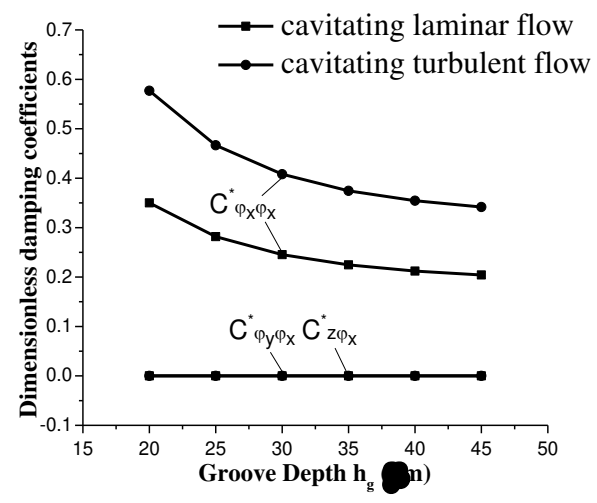

(b)

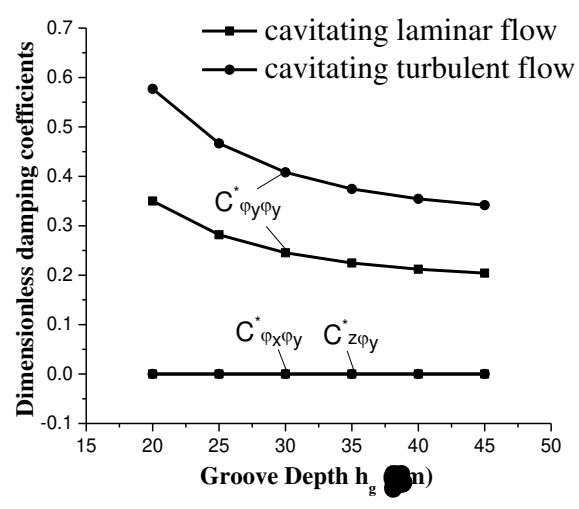

(c)

Fig. 18 Influence of turbulence effect on the damping coefficients for different groove depths.

$$
\left(\omega=18000 \mathrm{rpm}, \beta=20^{\circ}, h_{s}=15 \mu \mathrm{m}\right)
$$

Fig.19 shows the influence of turbulence effect on the water film damping coefficients for different rotating speed. It can be seen that the direct damping coefficients with turbulent cavitating flow are larger than those with the laminar 
cavitating flow at a specified rotating speed. And the deviation of water film damping coefficients between turbulence model and laminar model is also gradually increased with the increasing of rotating speed.

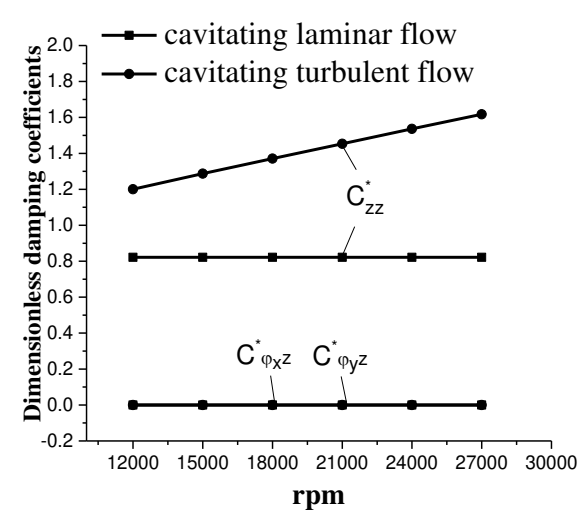

(a)

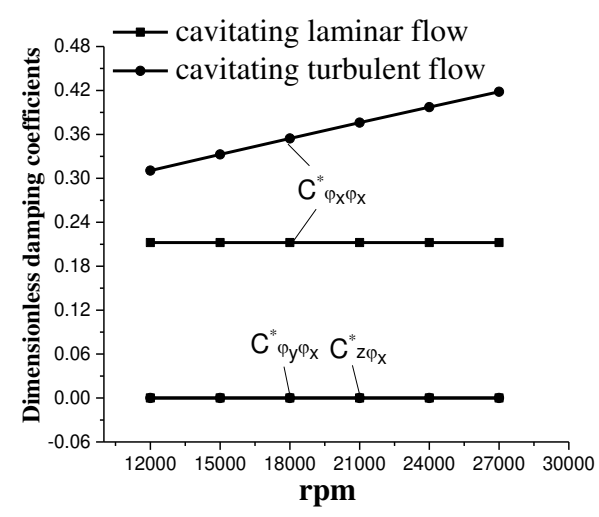

(b)

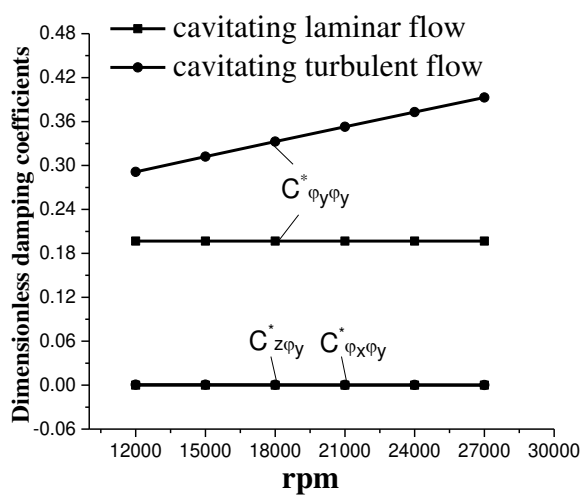

(c)

Fig. 19 Influence of turbulence effect on the damping coefficients for different rotating speed.

$$
\left(\beta=20^{\circ}, h_{s}=15 \mu m, h_{g}=40 \mu m\right)
$$

\section{3) Bubble density}

Fig. 20 shows the influence of turbulence effect on the bubble number for different radius. It can be seen that the bubbles mainly locate at the groove-ridge edges and the outer rim of bearing. This may be explained by the fact that the bubbles are formed due to the pressure drop near the groove-ridge edges. The result also show that the bubbles are approximately evenly distributed in dam region of bearing. In addition, the number 
of bubbles with laminar flow is more than the number of bubbles with turbulent flow at a specified circumferential position. Because the Reynolds shear stress due to the turbulent flow causes the equivalent viscosity of the turbulent liquid to increase, consequently, the turbulent flow effect on dynamic pressure is enhanced, which contributes to the generation of the bubbles.

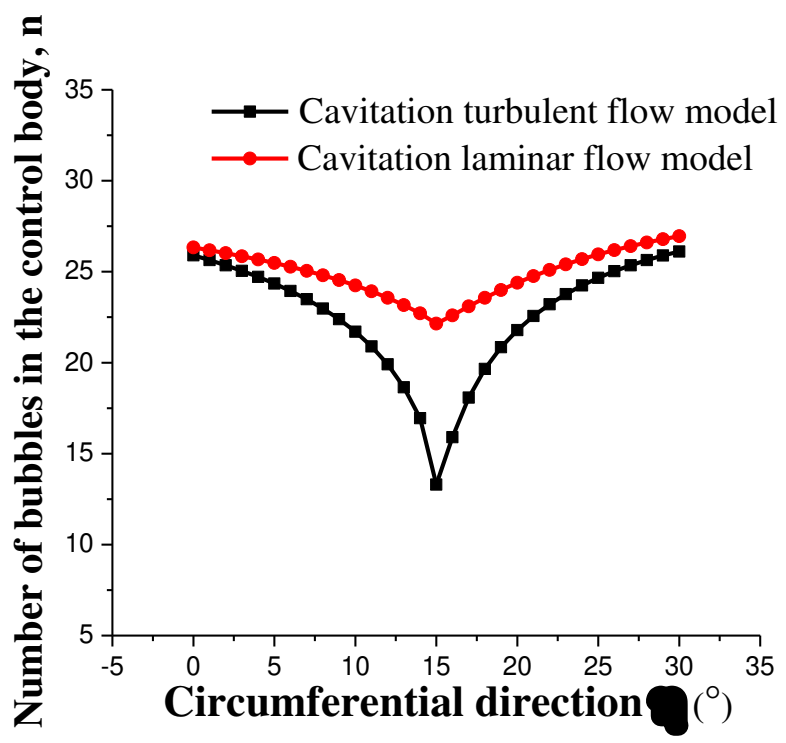

(a)

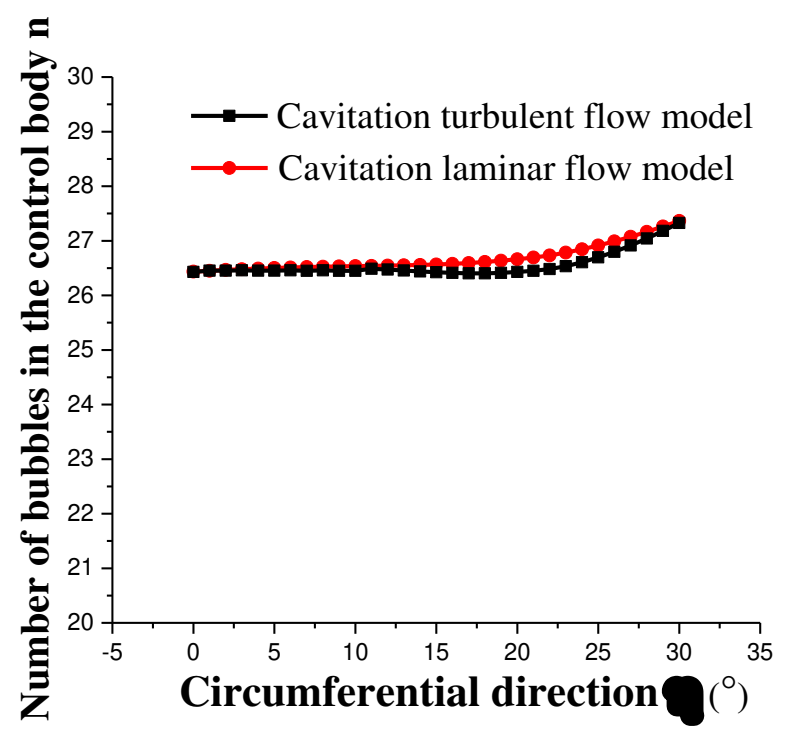

(b)

Fig. 20 Influence of turbulence effect on bubbles number for different

$$
\begin{gathered}
\text { radius.(a) } \zeta=0.2 \text { (b) } \zeta=0.5 \\
\left(\omega=18000 \mathrm{rpm}, \beta=20^{\circ}, h_{s}=15 \mu \mathrm{m}, h_{g}=40 \mu \mathrm{m}\right)
\end{gathered}
$$




\section{Conclusions}

In this paper, a theoretical model of water lubrication based on two-phase flow considering both turbulence and cavitation bubble effect is established. In the turbulent state, the evolution of the size distribution of the bubbles is described by the bubble transport equation considering the mechanism of breakage and coalescence. The interval discretization method is used to solve the bubble transport equation. The size and spatial equilibrium distribution of the bubbles under turbulent flow are predicted by solving the generalized Reynolds equation and the bubble transport equation simultaneously. The dynamic characteristics of water-lubricated SGTB under turbulent cavitating flow is analyzed. Based on discussion, the following conclusion can be drawn:

(1) The bubbles are mainly concentrated near the spiral-groove edges of the SGTB.

(2) The small-sized bubbles is much more than the large-sized bubbles for the waterlubricated SGTB under the cavitation flow condition.

(3) The cavitation has a significant effect on the direct stiffness coefficients, and the cavitation hardly affect the cross-coupled stiffness coefficients and the damping coefficients of SGTB.

(4) Compared to the cavitation, the turbulence has a much greater effect on the dynamic characteristics of the high-speed water-lubricated SGTB.

\section{Declaration}

\section{Funding}

The authors gratefully acknowledge the support provided by the National Science Foundation of China through grant Nos. 51635004, 11472078.

\section{Availability of data and materials}

The datasets supporting the conclusions of this article are included within the article.

\section{Authors' contributions}

The author' contributions are as follows: Xiaohui Lin was in charge of the whole trial; Xiaohui Lin wrote the manuscript; Shun Wang is responsible for model calculation and drawing;Shuyun Jiang revises and reviews the manuscript; Shaowen Zhang assisted with laboratory analyses. 


\section{Competing interests}

The authors declare no competing financial interests.

\section{Consent for publication}

Not applicable

\section{Ethics approval and consent to participate}

Not applicable

\section{Acknowledgements}

Not applicable

\section{Nomenclature}

$b=$ temperature viscosity coefficient $\left(\frac{1}{K}\right)$

$C_{i j}\left(i=z, \varphi_{x}, \varphi_{y} ; j=z, \varphi_{x}, \varphi_{y}\right)$ nine damping coefficients $\left(\left(\frac{N \cdot s}{\mathrm{~m}}, \frac{\mathrm{N} \cdot \mathrm{s}}{\mathrm{rad}}, N \cdot s, \frac{\mathrm{N} \cdot \mathrm{m} \cdot \mathrm{s}}{\mathrm{rad}}\right)\right)$

$C_{i j}^{*}\left(i=z, \varphi_{x}, \varphi_{y} ; j=z, \varphi_{x}, \varphi_{y}\right)$ nine dimensionless damping coefficients

(dimensionless)

$C_{w}=$ the water volume fraction (dimensionless)

$C_{D S}=$ drag coefficient (dimensionless)

$C_{g}=$ the bubble volume fraction (dimensionless)

$c_{v}=$ the specific heat capacity of water $\left(\frac{\mathrm{J}}{\mathrm{kg} \cdot \mathrm{K}}\right)$

$c_{d}=$ the specific heat capacity of thrust $\operatorname{disk}\left(\frac{\mathrm{J}}{\mathrm{kg} \cdot \mathrm{K}}\right)$

$c_{1}, c_{2}=$ experimental constant (dimensionless)

$f_{c}=$ the function of $z^{*}$ containing the parameter $h_{c}^{*}$ (dimensionless)

$f_{e q}(\eta, \varsigma)=$ the equilibrium distribution function of bubble diameter at $(\zeta, \eta)$ coordinates system $\left(\frac{1}{m^{6}}\right)$

$f(\eta, \varsigma)=$ the distribution function of bubble diameter at $(\zeta, \eta)$ coordinates system $\left(\frac{1}{m^{6}}\right)$ 
$g=$ gravity acceleration $\left(\frac{m}{s^{2}}\right)$

$g_{c}=$ the function of $z^{*}$ containing the parameter $h_{c}^{*}$ (dimensionless)

$h=$ the film thickness $(m)$

$h_{g}=$ the groove depth $(m)$

$h_{0}=$ Static film thickness $(m)$

$h_{s}=$ the film thickness of the ridge $(m)$

$h_{i}=$ initial film thickness of bubble $(m)$

$h_{f}=$ critical rupture film thickness of bubble $(m)$

$h_{c}^{*}=$ the shear stress parameter $h_{c}^{*}$ of the Couette flow (dimensionless)

$k_{s}=$ heat transfer coefficient of the stationary $\operatorname{ring}\left(\frac{\mathrm{W}}{\mathrm{m} \cdot \mathrm{K}}\right)$

$k_{d}=$ heat transfer coefficient of the thrust disk $\left(\frac{W}{m \cdot K}\right)$

$k_{w}=$ heat transfer coefficient of water $\left(\frac{W}{m \cdot K}\right)$

$k_{r}=$ the turbulence correction coefficient of radial direction (dimensionless)

$k_{\theta}=$ the turbulence correction coefficient of circumferential direction (dimensionless)

$K_{i j}\left(i=z, \varphi_{x}, \varphi_{y} ; j=z, \varphi_{x}, \varphi_{y}\right)$ nine stiffness coefficients $\left(\frac{N}{m}, \frac{N}{r a d}, N, \frac{N \cdot m}{r a d}\right)$

$K_{i j}^{*}\left(i=z, \varphi_{x}, \varphi_{y} ; j=z, \varphi_{x}, \varphi_{y}\right)$ nine dimensionless stiffness coefficients

(dimensionless)

$l=$ the circumferential discrete node(dimensionless)

$M(\eta)=$ the interface item at $(\zeta, \eta)$ coordinates system $\left(\frac{N}{m^{3}}\right)$

$N=$ the number of spiral groove (dimensionless)

$N_{i}=$ the number density of nuclei $\left(\frac{1}{m^{3}}\right)$

$n_{e q}^{k}(\eta)=$ the number of bubbles in the $k$ th volume range $\left(\varsigma_{k}, \varsigma_{k+1}\right)$ at equilibrium 
(dimensionless)

$n_{k}(\eta)=$ the number of bubbles in the $k$ th volume range $\left(\varsigma_{k}, \varsigma_{k+1}\right)$ (dimensionless)

$p=$ the water film pressure $\left(\frac{N}{m^{2}}\right)$

$p_{0}=$ the static pressure $\left(\frac{N}{m^{2}}\right)$

$p_{z}, p_{\varphi_{x}}, p_{\varphi_{y^{\prime}}} p_{\dot{z}}, p_{\dot{\varphi}_{x}}, p_{\dot{\varphi}_{y}}=$ perturbed pressure components $\left(\frac{N}{m^{3}}, \frac{N}{m^{2}}, \frac{N \cdot s}{m^{3}}, \frac{N \cdot s}{m^{2}}\right)$

$p_{v}=$ the vaporization pressure of water $\left(\frac{N}{m^{2}}\right)$

$p_{i n}=$ the pressure at inter radius $\left(\frac{N}{m^{2}}\right)$

$Q_{i}^{\eta}, Q_{j}^{\zeta}=$ the volume flow $\left(\frac{m^{3}}{s}\right)$

$r=$ the radial coordinate in polar coordinates $(m)$

$r_{b}=$ the base circle radius of spiral line $(m)$

$r_{i n}=$ thrust disk inner radius $(m)$

$r_{\text {out }}=$ thrust disk outer radius $(m)$

$R_{b}=$ the statistical average radius of bubbles $(m)$

$R_{e}=$ Reynolds number (dimensionless)

$S_{c}=$ the source terms $\left(\frac{1}{m^{6} s}\right)$

$t=$ the time $(s)$

$T=$ Water film temperature $(K)$

$T_{m}=$ the average temperature of water film $(K)$

$T_{S}=$ the temperature of stationary $\operatorname{ring}(K)$

$T_{d}=$ the temperature of thrust plate $(K)$

$T_{0}=$ the ambient temperature $(K)$

$U=$ the max circumferential velocity of thrust disk $\left(\frac{m}{s}\right)$

$\bar{u}=$ the circumferential average velocity of water $\left(\frac{m}{s}\right)$ 
$u=$ the circumferential velocity of water at $(\zeta, \eta)$ coordinates system $\left(\frac{m}{s}\right)$

$u_{b}=$ the bubble velocity $\left(\frac{m}{s}\right)$

$x_{k}=$ the diameter of bubbles $(m)$

$z=$ the coordinate of the film thickness $(m)$

$z^{*}=$ the coordinate of the dimensionless film thickness $\left(z^{*}=\frac{z}{h}\right)$

$z_{s}=$ the coordinate of thickness of the stationary ring $(\mathrm{m})$

$z_{d}=$ the coordinate of thickness of the thrust disk $(m)$

\section{Greek Letters}

$\alpha=$ experimental constant (dimensionless)

$\alpha_{a}=$ air convection heat transfer coefficient $\left(\frac{W}{m^{2} \cdot K}\right)$

$\alpha_{w}=$ forced convective heat transfer coefficient of water $\left(\frac{W}{m^{2} \cdot K}\right)$

$\beta=$ the helix angle of bearing $(\mathrm{deg})$

$\beta_{c}=$ the parameter in coalescence kernel (dimensionless)

$\delta=$ thickness coordinate of stationary ring $(m)$

$\delta_{s}=$ the thickness of the stationary $\operatorname{ring}(\mathrm{m})$

$\delta_{d}=$ the thickness of thrust plate $(m)$

$\zeta=$ the spiral coordinates $(m)$

$\zeta_{b}=$ the base circle radius of spiral line at $(\zeta, \eta)$ coordinates system $(m)$

$\zeta_{\text {in }}=$ radius of the oil inlet at $(\zeta, \eta)$ coordinates system $(m)$

$\zeta_{\text {out }}=$ radius of the oil outlet at $(\zeta, \eta)$ coordinates system $(m)$

$\varepsilon=$ turbulent kinetic energy dissipation rate per unit mass $\left(\frac{m^{2}}{s^{3}}\right)$

$\eta=$ the spiral coordinates $(\mathrm{deg})$ 


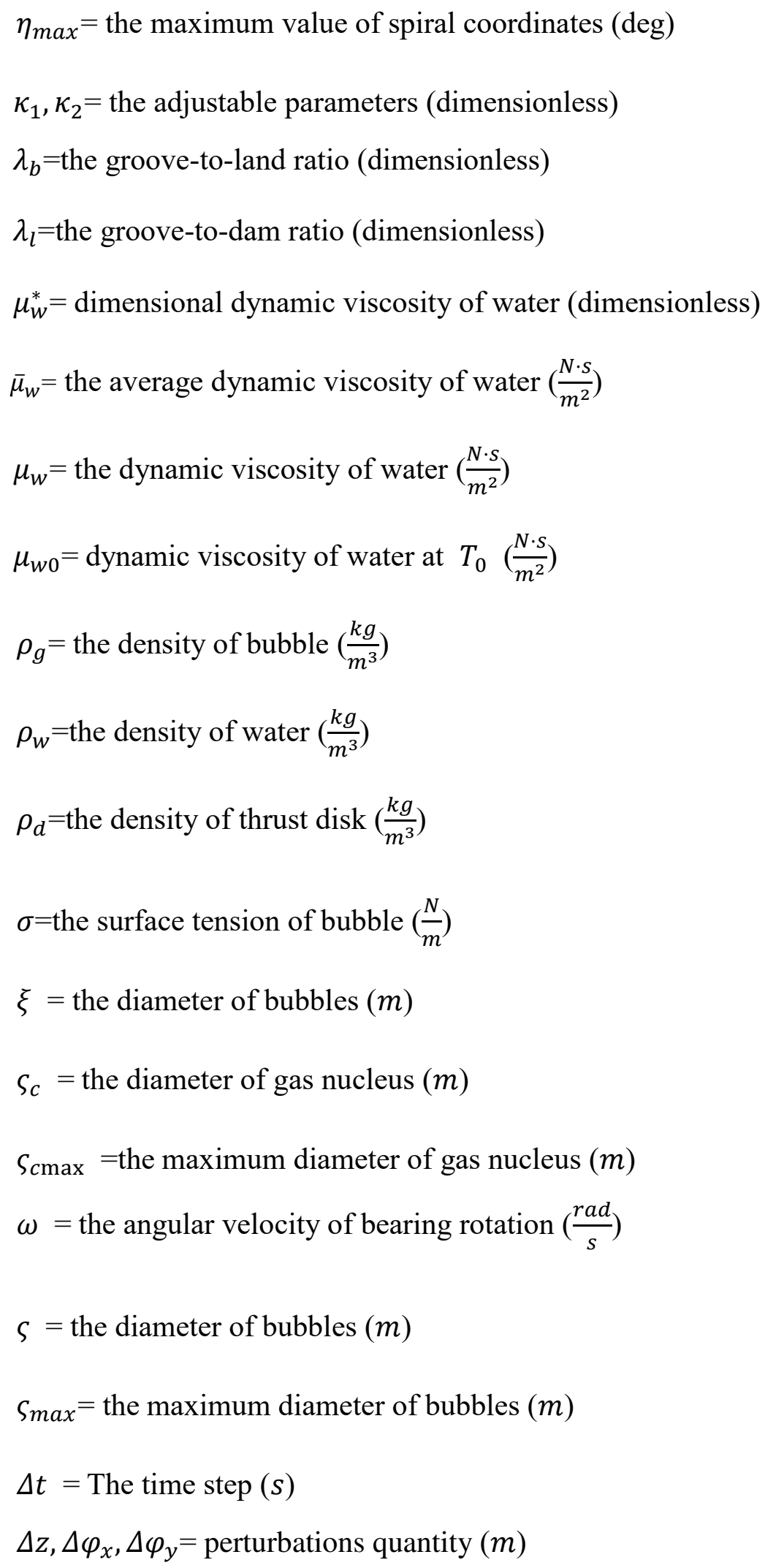

\section{References}

[1] B C Majumdar, R Pai, D J Hargreaves. Analysis of water-lubricated journal 
bearings with multiple axial grooves. Proc Inst Mech Eng Part J: J Eng Tribol, 2004, 218: 135-146.

[2] Y M Zhao, C Wei, S Yuan, et al. Theoretical and Experimental Study of Cavitation Effects on the Dynamic Characteristic of Spiral-Groove Rotary Seals(SGRSs). Tribology letters, 2016, 64(3): 1-18.

[3] H G Elrod. A Cavitation Algorithm. ASME J. Lubr. Technol,1981, 103(3): 350354.

[4] Y Song, C Gu. Development and validation of a three-dimensional computational fluid dynamics analysis for journal bearings considering cavitation and conjugate heat transfer. Journal of Engineering for Gas Turbines and Power, 2015, 137(12):122502.

[5] Y Wang, Z Yin, D Jiang, G Gao, et al. Study of the lubrication performance of waterlubricated journal bearings with CFD and FSI method. Industrial Lubrication and Tribology, 2016, 68(3): 341-348.

[6] E P Grando, M Priest, A T Prata. A two-phase flow approach to cavitation modelling in journal bearings. Tribology Letters, 2006, 21(3): 233-244.

[7] $\mathrm{H}$ Liu, $\mathrm{H} \mathrm{Xu}, \mathrm{P}$ J Ellison, et al. Application of computational fluid dynamics and fluid- structure interaction method to the lubrication study of a rotor-bearing system. Tribology Letters, 2010, 38(3): 325-336.

[8] J L Nikolajsen. The Effect of Aerated Oil on the Load Capacity of a Plain Journal Bearing. Tribology Transactions, 1999, 42(1): 58-62.

[9] S Choi, K W Kim. Analysis of Bubbly Lubrication in Journal Bearings. JSME International Journal Series C, 2002, 45(3): 802-808.

[10] Q Li, S L Liu, X H Pan, et al. A new method for studying the 3D transient flow of misaligned journal bearings in flexible rotor-bearing systems. Journal of Zhejiang University Science A, 2012, 13(4): 293-310.

[11] Q Li, G Yu, S Liu, S Zheng. Application of computational fluid dynamics and fluid structure interaction techniques for calculating the 3D transient flow of journal bearings coupled with rotor systems. Chinese Journal of Mechanical Engineering, 2012, 25(5): 926-932. 
[12] J Hu, W Wei, M Wu, S Yuan. Numerical investigation of the air-oil two-phase flow inside an oil-jet lubricated ball bearing. International Journal of Heat and Mass Transfer, 2014, 68: 85-93.

[13] X Zhang, Z Yin, G Gao, Z Li. Determination of stiffness coefficients of hydrodynamic water-lubricated plain journal bearings. Tribology International., 2015, 85: 37-47.

[14] Q Li, S Zhang, L Ma, W Xu, S Zheng. Stiffness and damping coefficients for journal bearing using the 3D transient flow calculation. Journal of Mechanical Science and Technology, 2017, 31(5): 2083-2091.

[15] Y Chen, Y Sun, Q He, J Feng. Elastohydrodynamic Behavior Analysis of Journal Bearing Using Fluid-Structure Interaction Considering Cavitation," Arabian Journal for Science and Engineering, 2019, 44: 1305-1320.

[16] D Sun, S Y Li, C W Fei, et al. Investigation of the effect of cavitation and journal whirl on static and dynamic characteristics of journal bearing. Journal of Mechanical Science and Technology, 2019, 33(1): 77-86.

[17] $\mathrm{H} \mathrm{Liu,} \mathrm{H} \mathrm{Xu}, \mathrm{Y}$ Zhang. The influence of sea water in oil emulsion on bearing performance. Part J: Journal of Engineering Tribology, 2009, 223(3): 457-468.

[18] Q Y Lin, Z Y Wei, N Wang, W Chen. Analysis on the lubrication performances of journal bearing system using computational fluid dynamics and fluid-structure interaction considering thermal influence and cavitation. Tribology International, 2013, 64: 8-15.

[19] S B Shenoy, R Pai, D Rao, R B Pai. Elasto-hydrodynamic lubrication analysis of full 360 journal bearing using CFD and FSI techniques. World Journal of Modelling and Simulation, 2009, 5(4).

[20] X H Lin, S Y Jiang, C B Zhang. Thermohydrodynamic analysis of high speed water-lubricated spiral groove thrust bearing considering effects of cavitation, inertia and turbulence. Tribology International, 2018, 119: 645-658.

[21] X H Lin, S Y Jiang, C B Zhang, X Liu. Thermohydrodynamic Analysis of HighSpeed Water-Lubricated Spiral Groove Thrust Bearing Using Cavitating Flow Model. Journal of Tribology, 2018, 140: 0517031-05170312.

[22] X H Lin, R Q Wang, S W Zhang, C B Zhang, S Y Jiang. Study of Cavitation Bubbles Evolution for High-Speed Water-Lubricated Spiral Groove Thrust Bearings. ASME Journal of Tribology, 2019, 141: 051703-1.

[23] X H Lin, R Q Wang, S W Zhang, S Y Jiang. Study on dynamic characteristics for 
high speed water-lubricated spiral groove thrust bearing considering cavitating effect. Tribology International, 2019, 143:106022.

[24] C W Ng, C H T Pan. A Linearized Turbulent Lubrication Theory. ASME J. Fluids Eng, 1965, 87(3): 675-688.

[25] J O Hinze. Turbulence. McGraw-Hili Book Company., Inc., New York, 1959.

[26] J R Ni, G Q Wang, H W Zhang. The Basic Theory of Solid Liquid Two-Phase Flow and Its Latest Applications. Science Press, 1991.

[27] L J Guo. Two-Phase and Multiphase Flow Dynamics. Xi'an Jiaotong University Press, 2002.

[28] J Solsvik, H A Jakobsen. Evaluation of Weighted Residual Methods for the Solution of a Population Balance Model Describing Bubbly Flows: The Least Squares, Galerkin, Tau, and Orthogonal Collocation Methods. Industrial \& Engineering Chemistry Research, 2013, 52(45): 15988-16013.

[29] M L Billet. Cavitation Nuclei Measurements. In: New Orleans, Louisiana, USA. Proc. of 2nd Intcrn Symp on Cavitation Inception, 1984, 33-42.

[30] S Kumar, D Ramkrishna. On The Solution of Population Balance Equations By Discretization--I. A Fixed Pivot Technique. Chemical Engineering Science, 1996, 51(8): 1311-1332.

[31] C A Dorao, H A Jakobsen. A Least Squares Method for The Solution of Population Balance Problems. Computers and Chemical Engineering, 2006, 30: 535-547. 


\section{Appendix A. The derivation of perturbation generalized Reynolds equations}

The generalized Reynolds equation in $(r, \theta)$ coordinate system,

$$
\begin{gathered}
\frac{\partial}{\partial r}\left(\frac{h^{3} r}{\mu_{m} k_{r}}\left(\frac{\partial C_{w} p}{\partial r}\right)\right)+\frac{\partial}{\partial \theta}\left(\frac{h^{3}}{\mu_{m} k_{\theta} r}\left(\frac{\partial C_{w} p}{\partial \theta}\right)\right) \\
=\frac{\partial}{\partial \theta}\left(\frac{h U C_{w}}{2}+\frac{h^{3}}{\mu_{m} k_{\theta}} M(\theta)\right)+\frac{\partial}{\partial r}\left(\frac{h^{3} \rho_{w} \bar{u}^{2} C_{w}}{\mu_{m} k_{r}}\right)+C_{w} r \frac{\partial h}{\partial t}
\end{gathered}
$$

Ignoring the infinitesimal value of higher order, the expression of $h^{3}$ is as follows,

$$
h^{3} \approx h_{0}^{3}+3 h_{0}^{2}\left(\Delta z+r \cos \theta \Delta \varphi_{y}-r \sin \theta \Delta \varphi_{x}\right)
$$

Bring Eq. (A2) and Eq. (57) into the right side of Eq. (A1),

$$
\begin{gathered}
\frac{\partial}{\partial \theta}\left(\frac{h U C_{w}}{2}+\frac{h^{3}}{\bar{\mu}_{w} k_{\theta}} M(\theta)\right)+\frac{\partial}{\partial r}\left(\frac{h^{3} \rho_{w} \bar{u}^{2} C_{w}}{\bar{\mu}_{w} k_{r}}\right)+C_{w} r \frac{\partial h}{\partial t} \\
=\frac{\partial}{\partial \theta}\left(C_{w} U h_{0}\right)+\frac{\partial}{\partial \theta}\left(\frac{h_{0}^{3}}{\bar{\mu}_{w} k_{\theta}}\right)+\frac{\partial}{\partial r}\left(\frac{h_{0}^{3} \rho_{w} \bar{u}^{2} C_{w}}{\bar{\mu}_{w} k_{r}} h_{0}^{3}\right) \\
+\left[\frac{\partial}{\partial \theta}\left(C_{w} U\right)_{+} \frac{\partial}{\partial \theta}\left(\frac{M(\theta)}{\bar{\mu}_{w} k_{\theta}} 3 h_{0}^{2}\right)+\frac{\partial}{\partial r}\left(\frac{h^{3} \rho_{w} \bar{u}^{2} C_{w}}{\bar{\mu}_{w} k_{r}} 3 h_{0}^{2}\right)\right] \Delta z \\
+\left[\frac{\partial}{\partial \theta}\left(C_{w} U r \cos \theta\right)+\frac{\partial}{\partial \theta}\left(\frac{M(\theta)}{\bar{\mu}_{w} k_{\theta}} 3 h_{0}^{2} r \cos \theta\right)+\frac{\partial}{\partial r}\left(\frac{h^{3} \rho_{w} \bar{u}^{2} C_{w}}{\bar{\mu}_{w} k_{r}} 3 h_{0}^{2} r \cos \theta\right)\right] \Delta \varphi_{y} \\
-\left[\frac{\partial}{\partial \theta}\left(C_{w} U r \sin \theta\right)+\frac{\partial}{\partial \theta}\left(\frac{M(\theta)}{\bar{\mu}_{w} k_{\theta}} 3 h_{0}^{2} r \sin \theta\right)+\frac{\partial}{\partial r}\left(\frac{h^{3} \rho_{w} \bar{u}^{2} C_{w}}{\bar{\mu}_{w} k_{r}} 3 h_{0}^{2} r \sin \theta\right)\right] \Delta \varphi_{x} \\
+C_{w} r \Delta \dot{z}+C_{w} r^{2} \cos \theta \Delta \dot{\varphi}_{y}-C_{w} r^{2} \sin \theta \Delta \dot{\varphi}_{x}
\end{gathered}
$$

Convert the coordinate system of Eq. (A3) to the $(\zeta, \eta)$ coordinate system, and compare similar terms,

$$
\begin{gathered}
q_{z}=\frac{\partial}{\partial \eta}\left(C_{w} U\right)+\frac{\partial}{\partial \eta}\left(\frac{M(\theta)}{\mu_{w} k_{\theta}} 3 h_{0}^{2}\right)+\frac{\partial}{\partial \zeta}\left(\frac{\rho_{w} \bar{u}^{2} C_{w}}{\mu_{w} k_{r}} 3 h_{0}^{2}\right) \\
-\frac{\partial}{\partial \eta}\left(\frac{\rho_{w} \bar{u}^{2} C_{w}}{\zeta \mu_{w} k_{r} \cot \beta} 3 h_{0}^{2}\right)-\frac{\partial}{\partial \eta}\left(\frac{3 h_{0}^{2}}{\mu_{w} k_{\theta} \zeta} \frac{\partial C_{w} p_{0}}{\partial \eta}\right) \\
-\left[\begin{array}{c}
\frac{\partial}{\partial \zeta}\left(\frac{3 h_{0}^{2} \zeta}{\mu_{w} k_{r}} \frac{\partial C_{w} p_{0}}{\partial \zeta}\right)-\frac{\partial}{\partial \zeta}\left(\frac{3 h_{0}^{2}}{\cot \beta \mu_{w} k_{r}} \frac{\partial C_{w} p_{0}}{\partial \eta}\right) \\
-\frac{\partial}{\partial \eta}\left(\frac{3 h_{0}^{2}}{\cot \beta \mu_{w} k_{r}} \frac{\partial C_{w} p_{0}}{\partial \zeta}\right)+\frac{\partial}{\partial \eta}\left(\frac{3 h_{0}^{2}}{\zeta \cot ^{2} \beta \mu_{w} k_{r}} \frac{\partial C_{w} p_{0}}{\partial \eta}\right)
\end{array}\right] \\
q_{\varphi_{x}}=\frac{\partial}{\partial \eta}\left(C_{w} U G_{1}(\zeta, \eta)\right)+\frac{\partial}{\partial \eta}\left(\frac{M(\theta)}{\mu_{w} k_{\theta}} 3 h_{0}^{2} G_{1}(\zeta, \eta)\right)+\frac{\partial}{\partial \zeta}\left(\frac{\rho_{w} \bar{u}^{2} C_{w}}{\mu_{w} k_{r}} 3 h_{0}^{2} G_{1}(\zeta, \eta)\right) \\
-\frac{\partial}{\partial \eta}\left(\frac{\rho_{w} \bar{u}^{2} C_{w}}{\zeta \mu_{w} k_{r}} \frac{3 h_{0}^{2}}{\cot \beta} G_{1}(\zeta, \eta)\right)+\frac{\partial}{\partial \eta}\left(\frac{3 h_{0}^{2}}{\mu_{w} k_{\theta} \zeta} \frac{\partial C_{w} p_{0}}{\partial \eta} G_{1}(\zeta, \eta)\right) \\
+\left[\begin{array}{c}
\frac{\partial}{\partial \zeta}\left(\frac{3 h_{0}^{2} \zeta}{\mu_{w} k_{r}} \frac{\partial C_{w} p_{0}}{\partial \zeta} G_{1}(\zeta, \eta)\right)-\frac{\partial}{\partial \zeta}\left(\frac{3 h_{0}^{2}}{\cot \beta \mu_{w} k_{r}} \frac{\partial C_{w} p_{0}}{\partial \eta} G_{1}(\zeta, \eta)\right) \\
\left.-\frac{\partial}{\partial \eta}\left(\frac{3 h_{0}^{2}}{\cot \beta \mu_{w} k_{r}} \frac{\partial C_{w} p_{0}}{\partial \zeta} G_{1}(\zeta, \eta)\right)+\frac{\partial}{\partial \eta}\left(\frac{3 h_{0}^{2}}{\zeta \cot ^{2} \beta \mu_{w} k_{r}} \frac{\partial C_{w} p_{0}}{\partial \eta} G_{1}(\zeta, \eta)\right)\right]
\end{array}\right]
\end{gathered}
$$




$$
\begin{gathered}
q_{\varphi_{y}}=\frac{\partial}{\partial \eta}\left(C_{w} U G_{2}(\zeta, \eta)\right)+\frac{\partial}{\partial \eta}\left(\frac{M(\theta)}{\mu_{w} k_{\theta}} 3 h_{0}^{2} G_{2}(\zeta, \eta)\right)+\frac{\partial}{\partial \zeta}\left(\frac{\rho_{w} \bar{u}^{2} C_{w}}{\mu_{w} k_{r}} 3 h_{0}^{2} G_{2}(\zeta, \eta)\right) \\
-\frac{\partial}{\partial \eta}\left(\frac{\rho_{w} \bar{u}^{2} C_{w}}{\zeta \mu_{m} k_{r}} \frac{3 h_{0}^{2}}{\cot \beta} G_{2}(\zeta, \eta)\right)-\frac{\partial}{\partial \eta}\left(\frac{3 h_{0}^{2}}{\mu_{w} k_{\theta} \zeta} \frac{\partial C_{w} p_{0}}{\partial \eta} G_{2}(\zeta, \eta)\right) \\
-\left[\begin{array}{c}
\frac{\partial}{\partial \zeta}\left(\frac{3 h_{0}^{2} \zeta}{\mu_{w} k_{r}} \frac{\partial C_{w} p_{0}}{\partial \zeta} G_{2}(\zeta, \eta)\right)-\frac{\partial}{\partial \zeta}\left(\frac{3 h_{0}^{2}}{\cot \beta \mu_{w} k_{r}} \frac{\partial C_{w} p_{0}}{\partial \eta} G_{2}(\zeta, \eta)\right) \\
\left.-\frac{\partial}{\partial \eta}\left(\frac{3 h_{0}^{2}}{\cot \beta \mu_{w} k_{r}} \frac{\partial C_{w} p_{0}}{\partial \zeta} G_{2}(\zeta, \eta)\right)+\frac{\partial}{\partial \eta}\left(\frac{3 h_{0}^{2}}{\zeta \cot ^{2} \beta \mu_{w} k_{r}} \frac{\partial C_{w} p_{0}}{\partial \eta} G_{2}(\zeta, \eta)\right)\right] \\
q_{\dot{z}}=C_{w} \zeta \\
q_{\dot{\varphi}_{x}}=-C_{w} \zeta G_{1}(\eta, \zeta) \\
q_{\dot{\varphi}_{y}}=C_{w} \zeta G_{2}(\eta, \zeta)
\end{array}\right.
\end{gathered}
$$

where

$$
\begin{aligned}
& G_{1}(\eta, \zeta)=\zeta \sin (\eta+f(\zeta)) \\
& G_{2}(\eta, \zeta)=\zeta \cos (\eta+f(\zeta))
\end{aligned}
$$


Figures
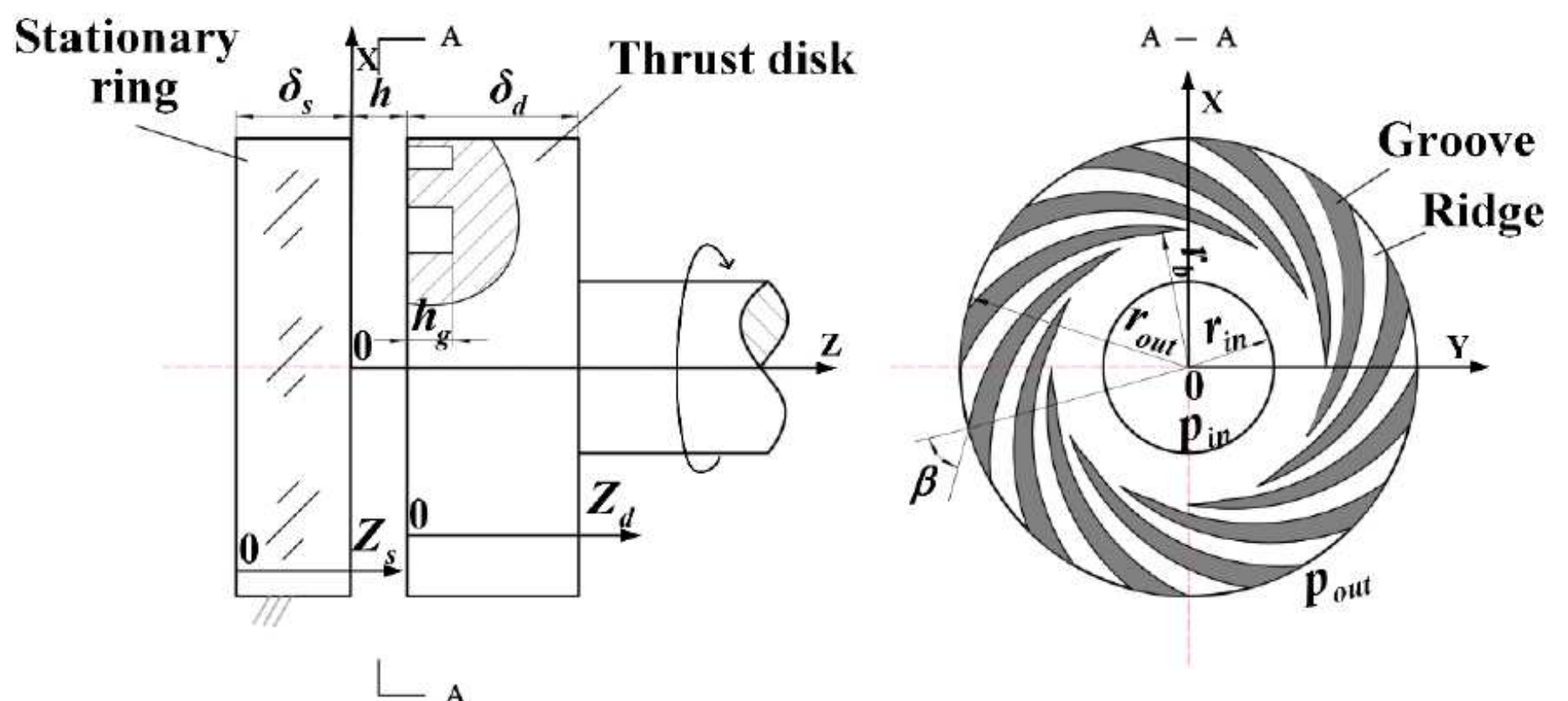

Figure 1

Structure schematic of inward-pumping SGTB
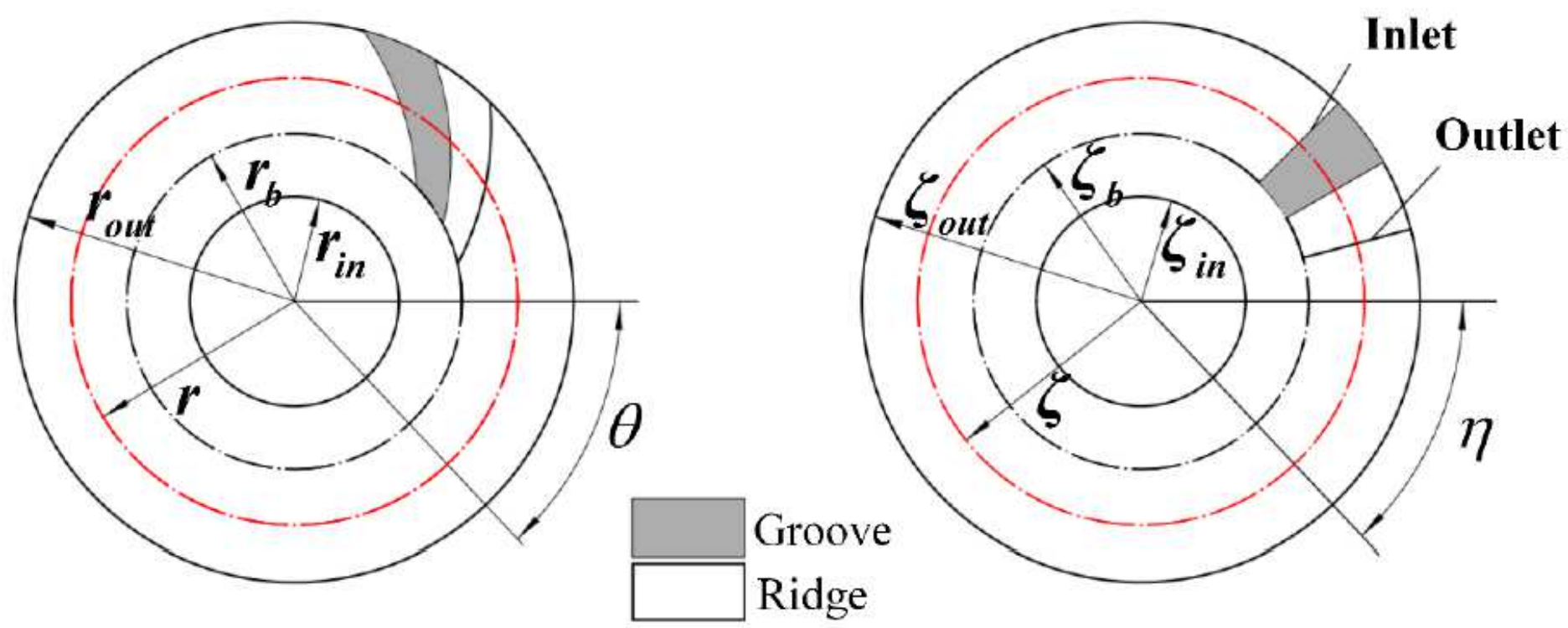

Figure 2

Coordinates transformation of spiral patterns 


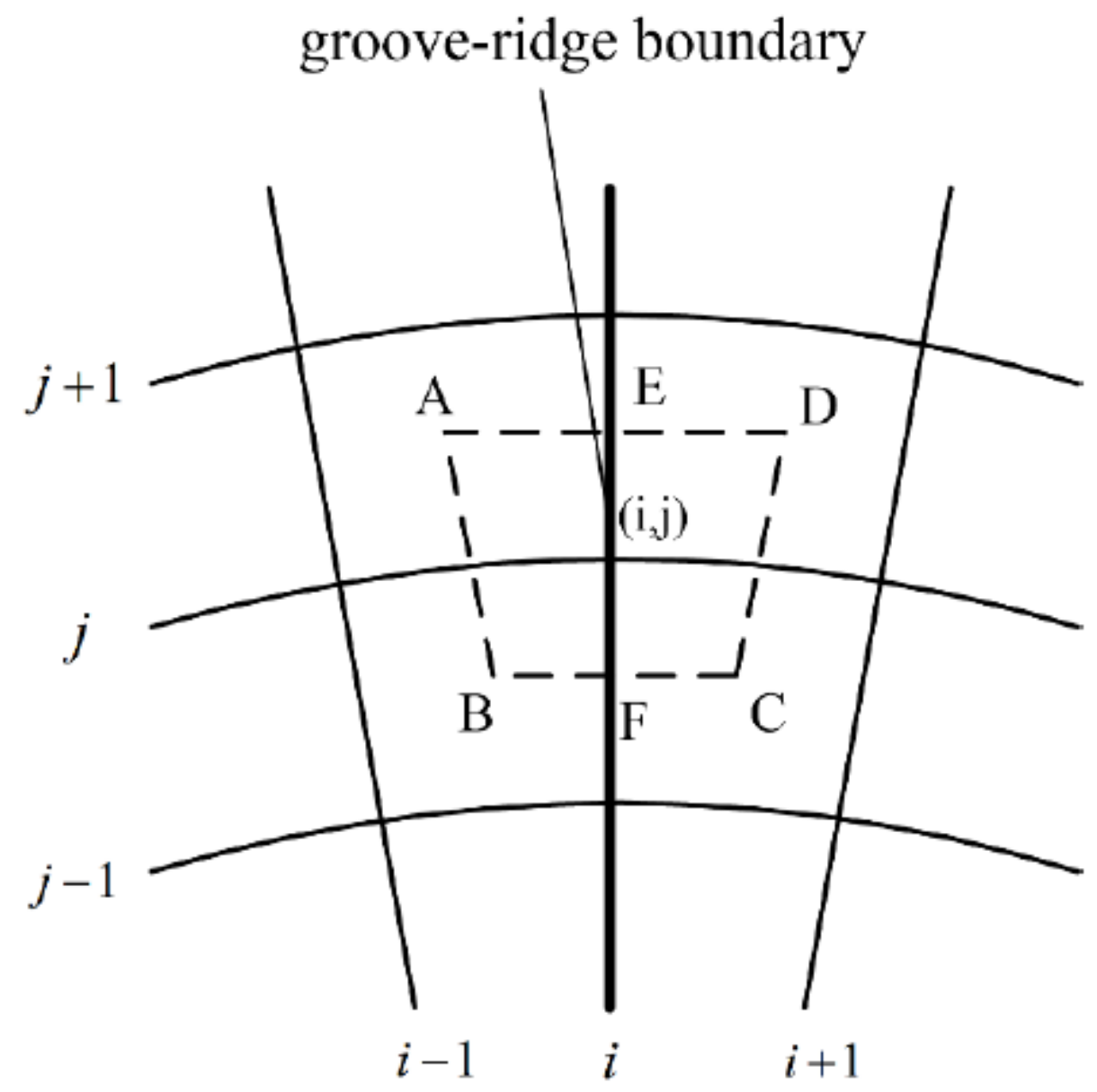

Figure 3

Finite volume separated by groove-ridge boundary 

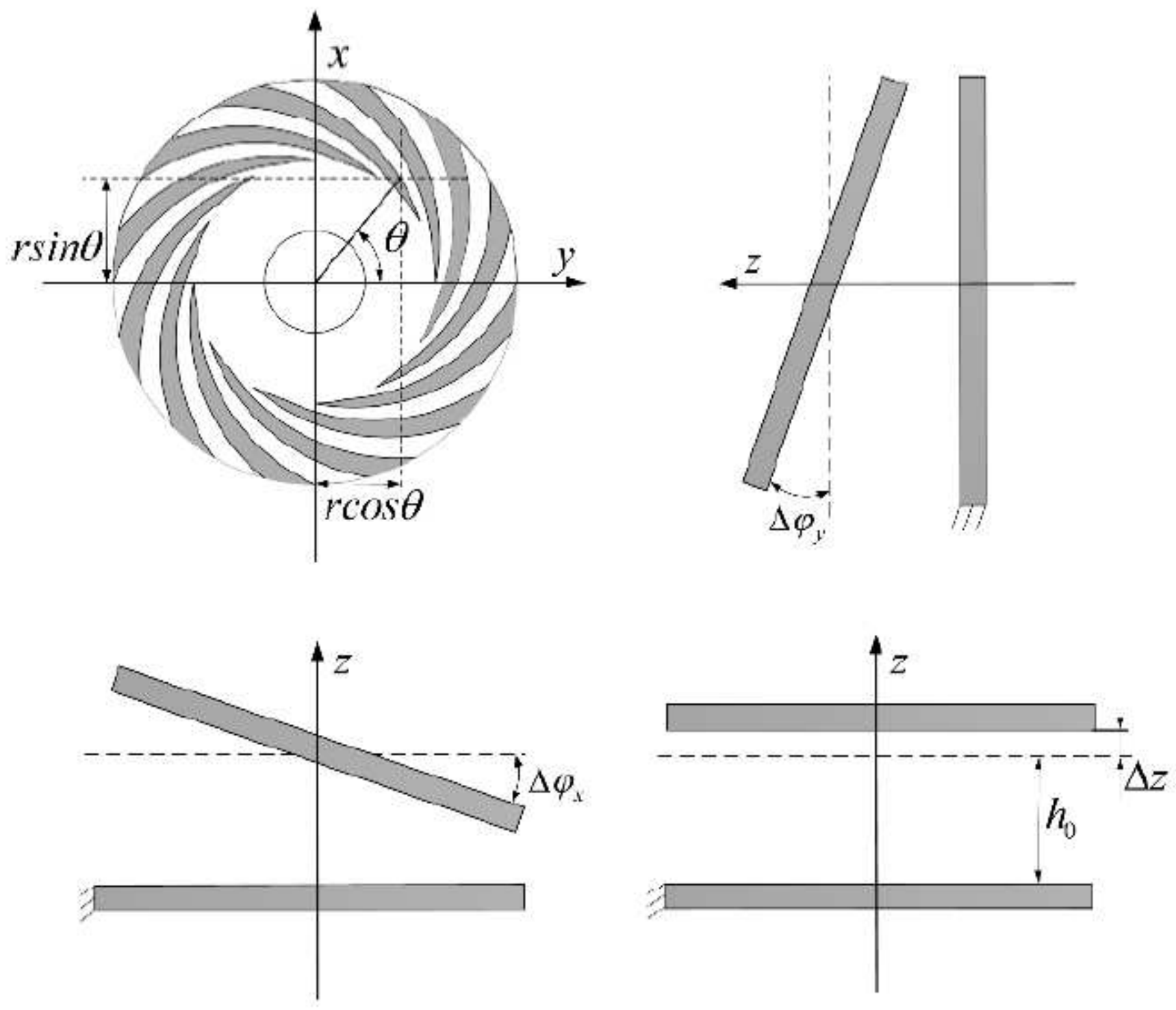

Figure 4

Motion of a SGTB 


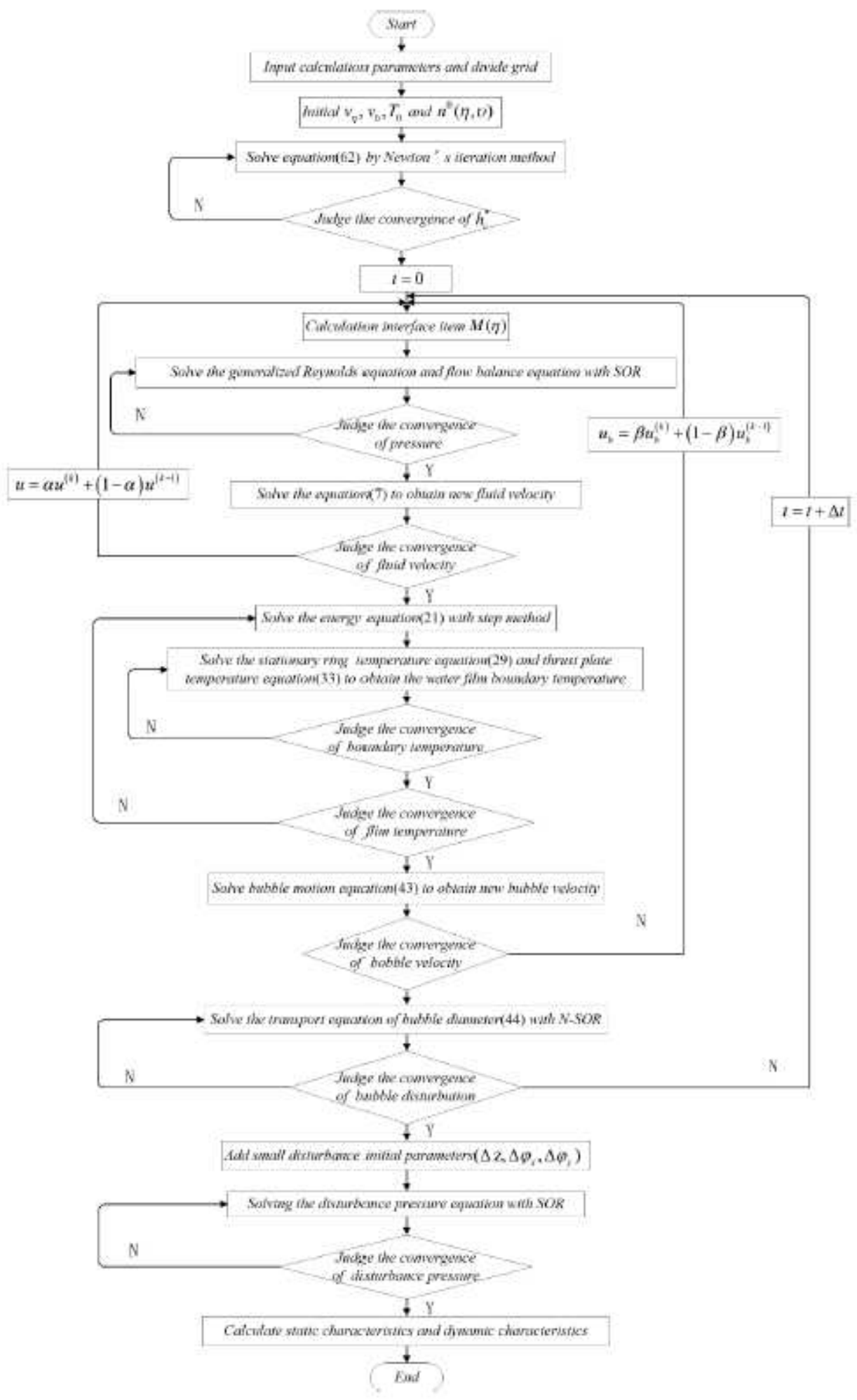

Figure 5

Flow diagram of overall algorithm 


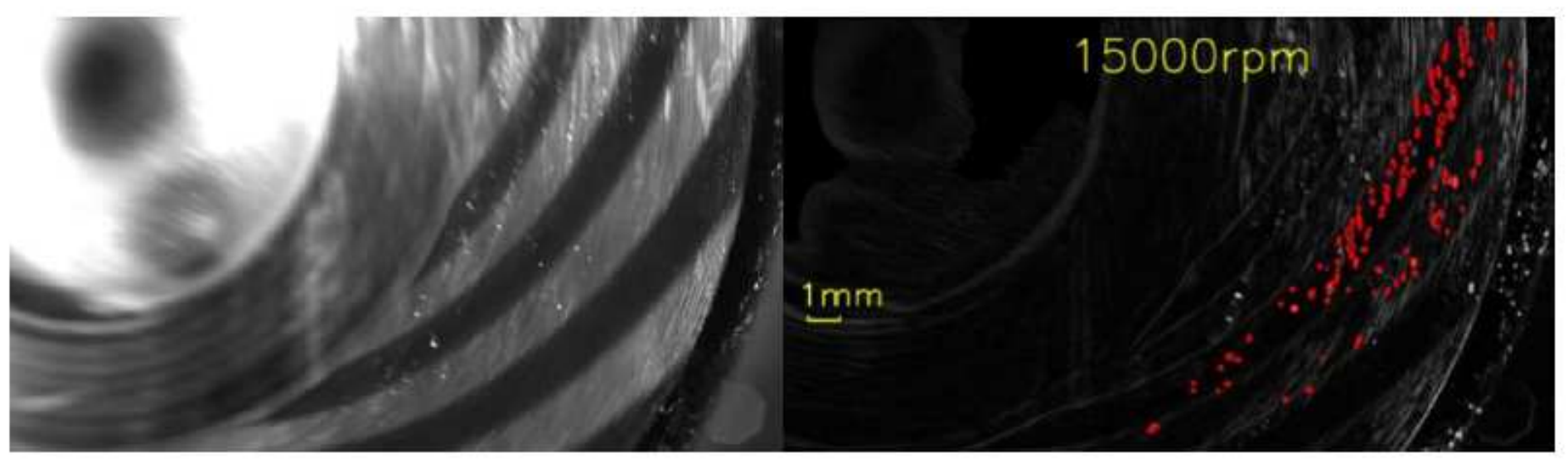

(a) $15000 \mathrm{rpm}$

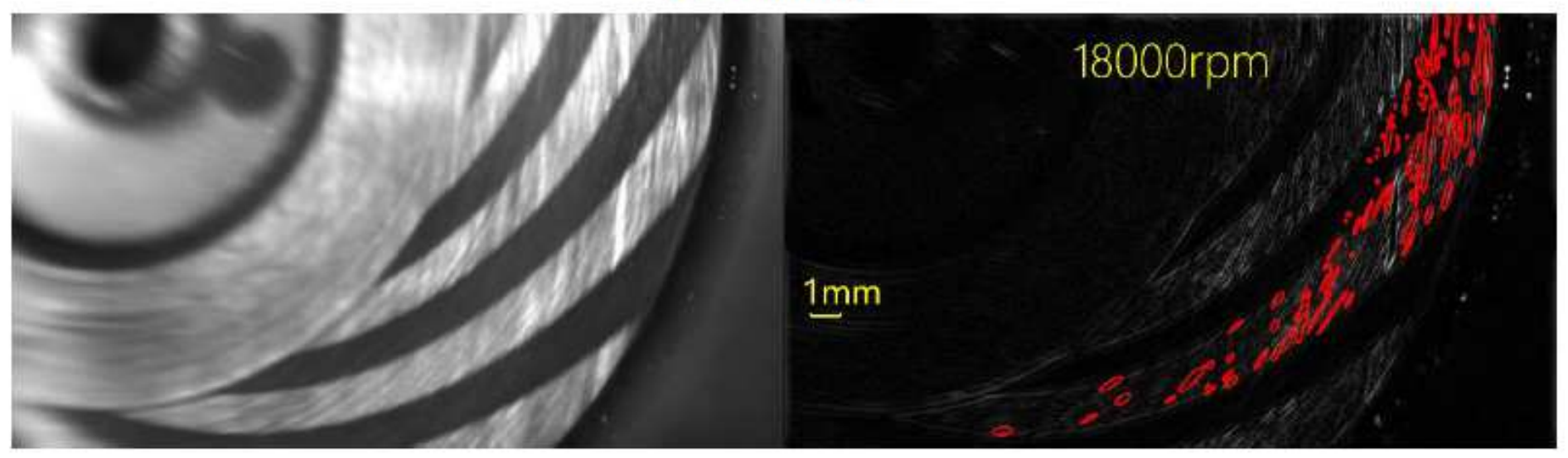

(b) $18000 \mathrm{rpm}$

Figure 6

Photographs of bubble distributions under both rotational speeds: (a) $15000 \mathrm{rpm}$, (b) $18000 \mathrm{rpm}$ 


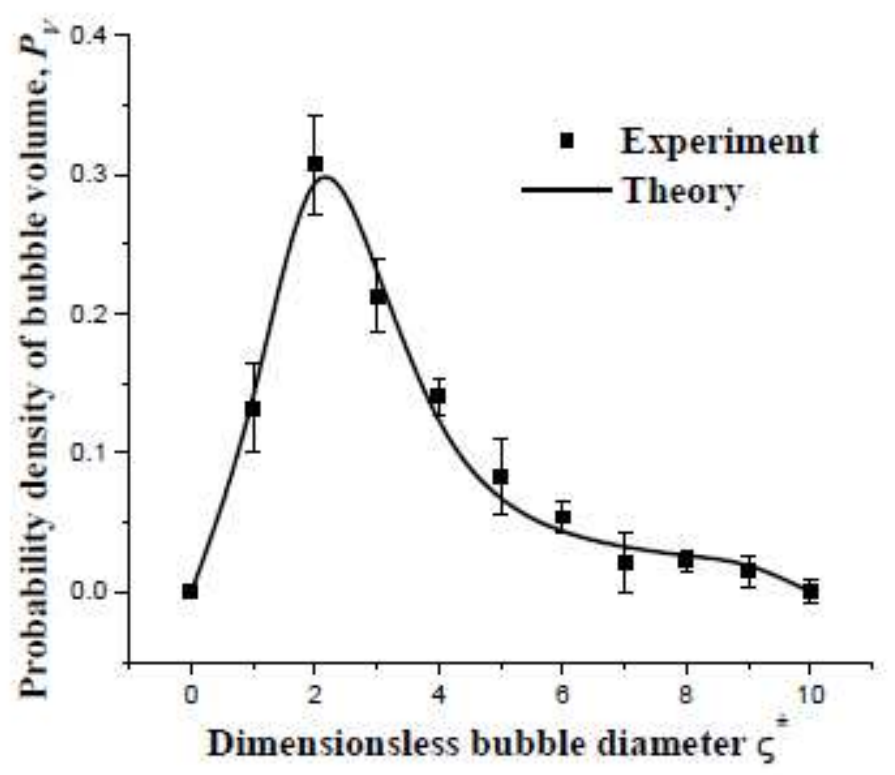

(a) $15000 \mathrm{rpm}$

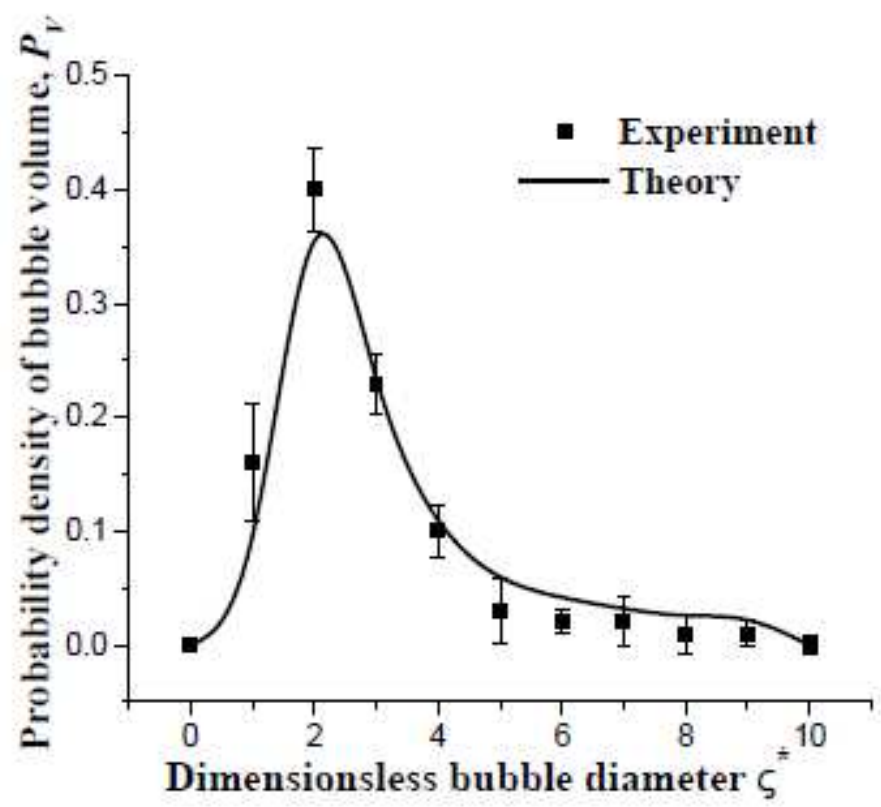

(b) $18000 \mathrm{rpm}$

\section{Figure 7}

Comparison of the theoretical probability density distributions with the experimental ones: (a) $15000 \mathrm{rpm} \rrbracket$ (b) $18000 \mathrm{rpm}$ 


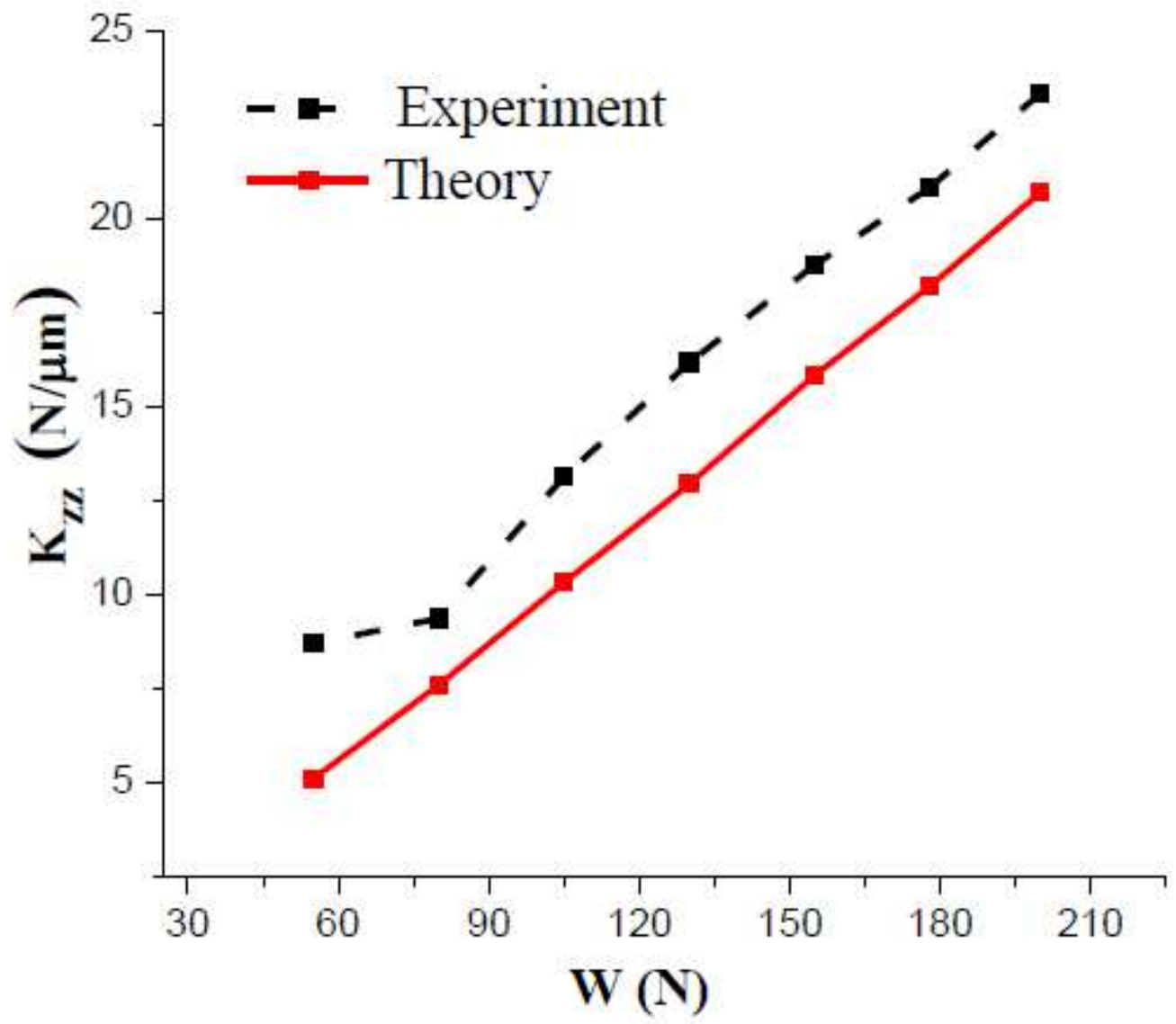

Figure 8

Comparison of the theoretical stiffness coefficients with the experimental ones at $15000 \mathrm{rpm}$ 


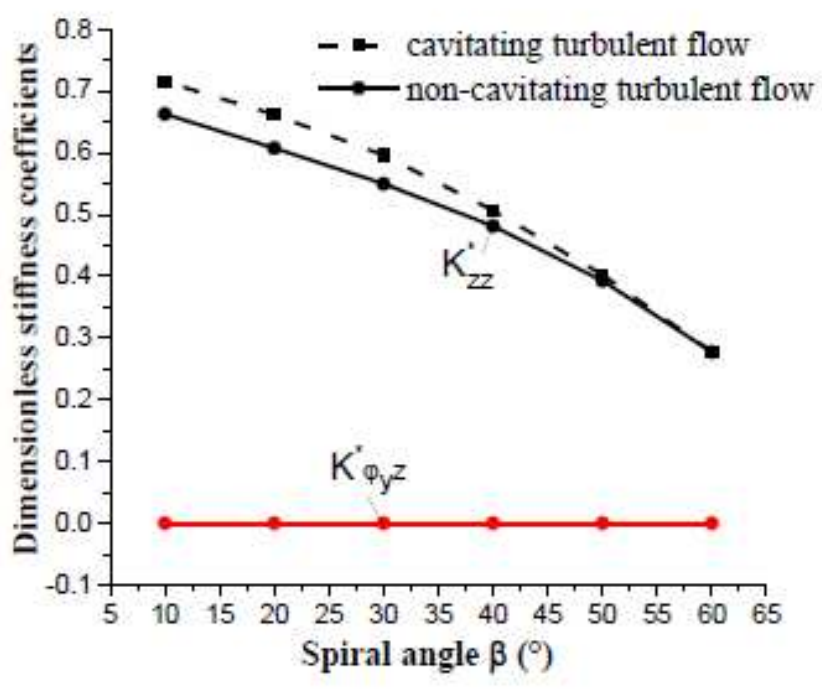

(a)

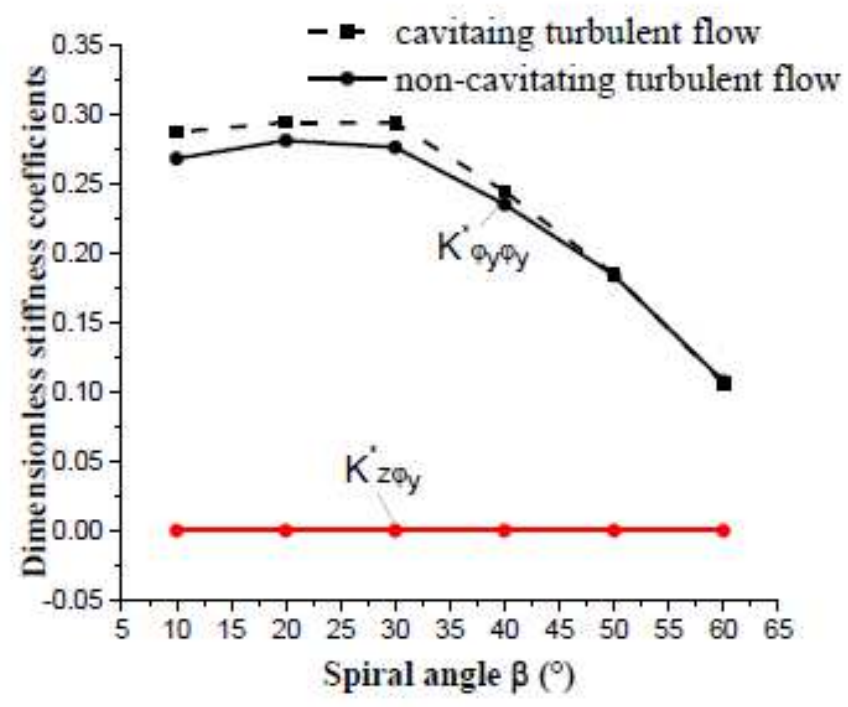

(c)

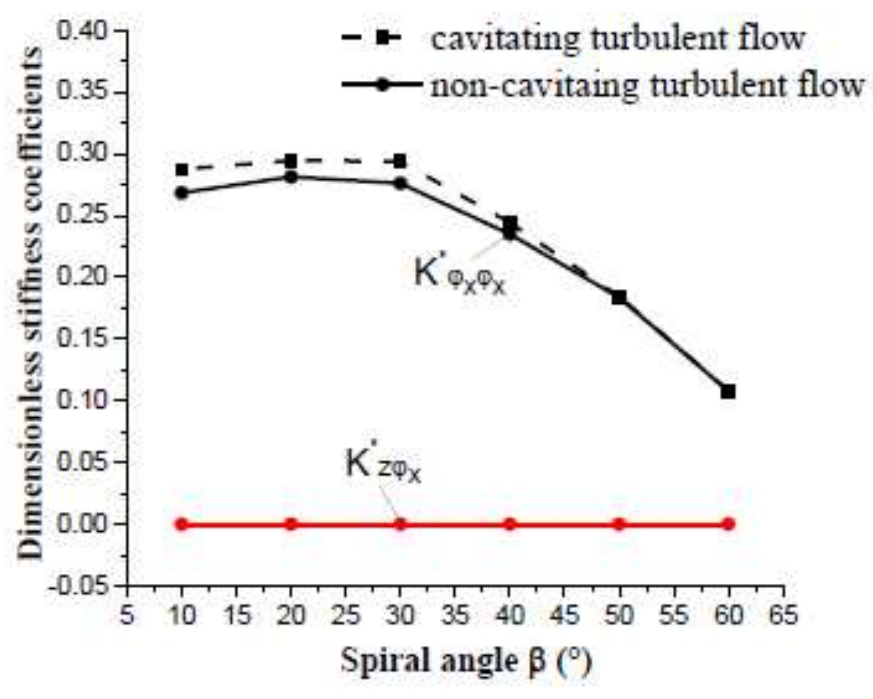

(b)

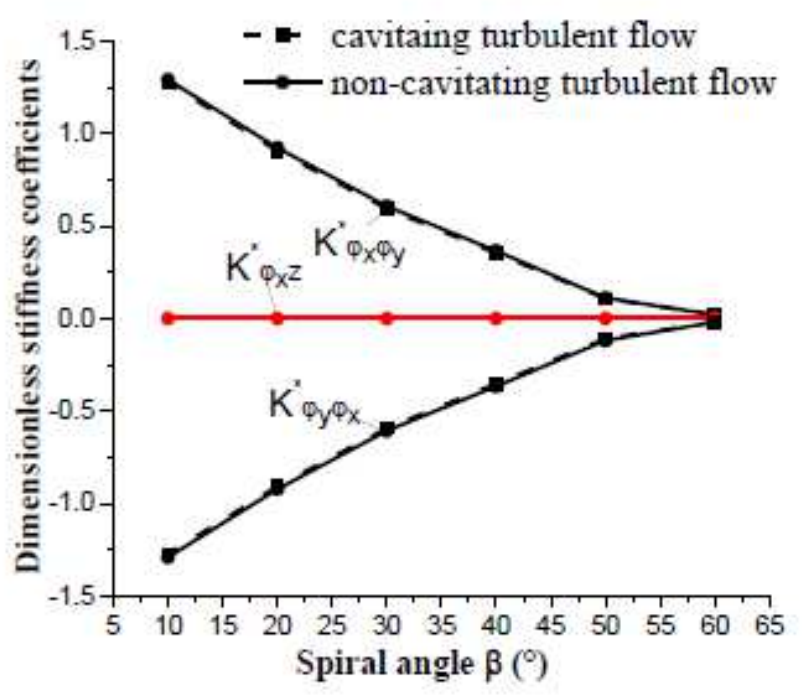

(d)

\section{Figure 9}

Influence of cavitation effect on the stiffness coefficient for different spiral angles 


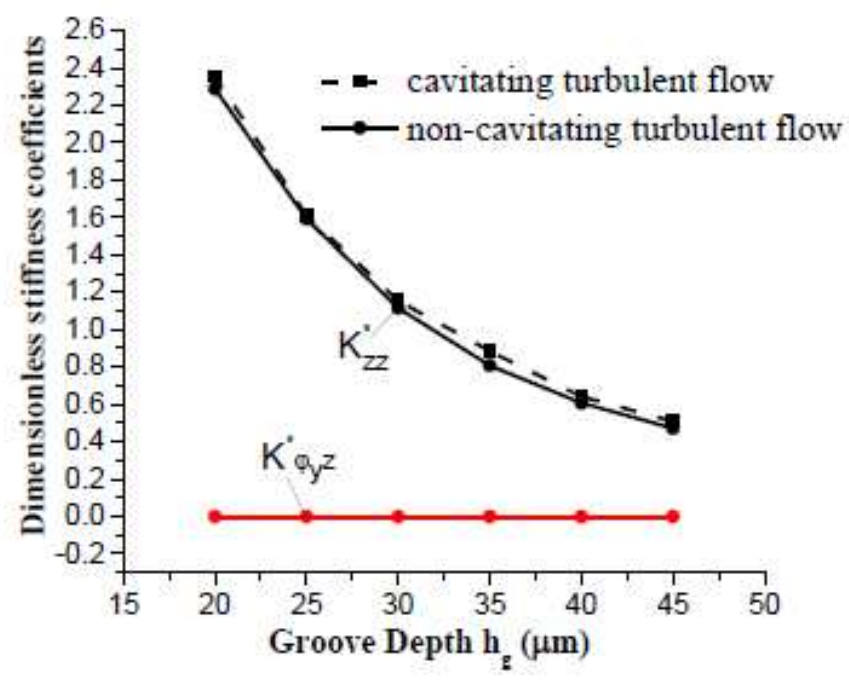

(a)

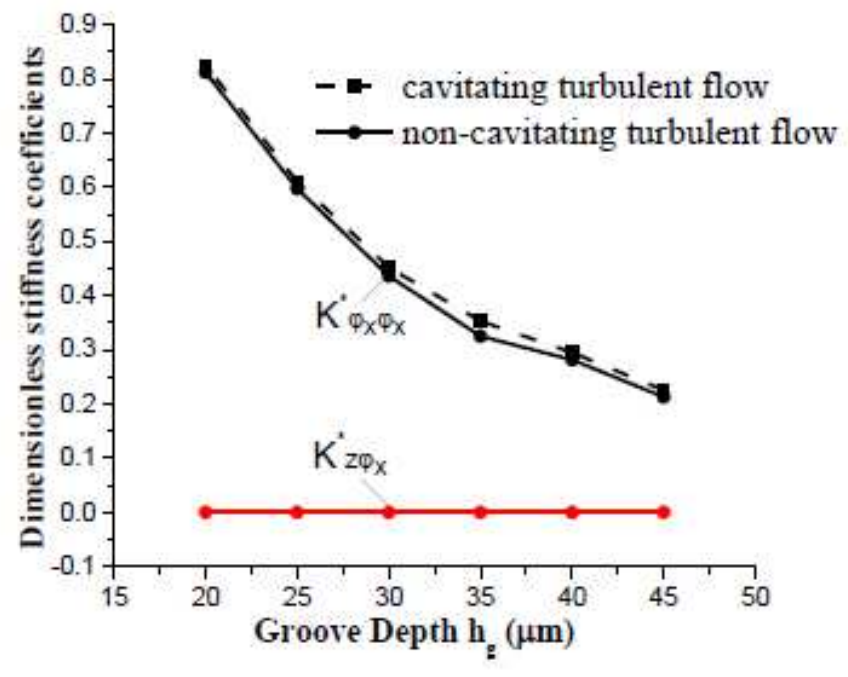

(b)

Figure 10

Influence of cavitation effect on stiffness coefficients for different groove depth 


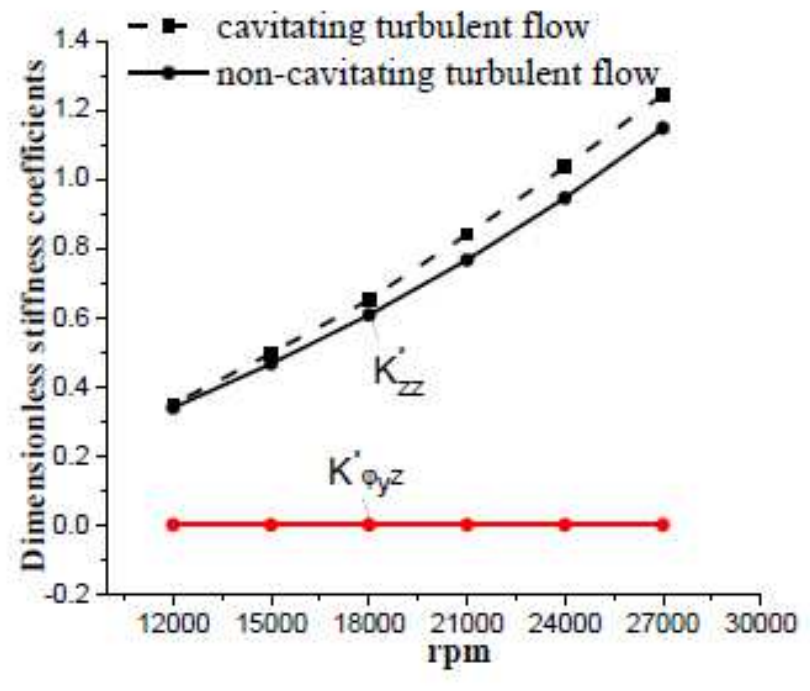

(a)

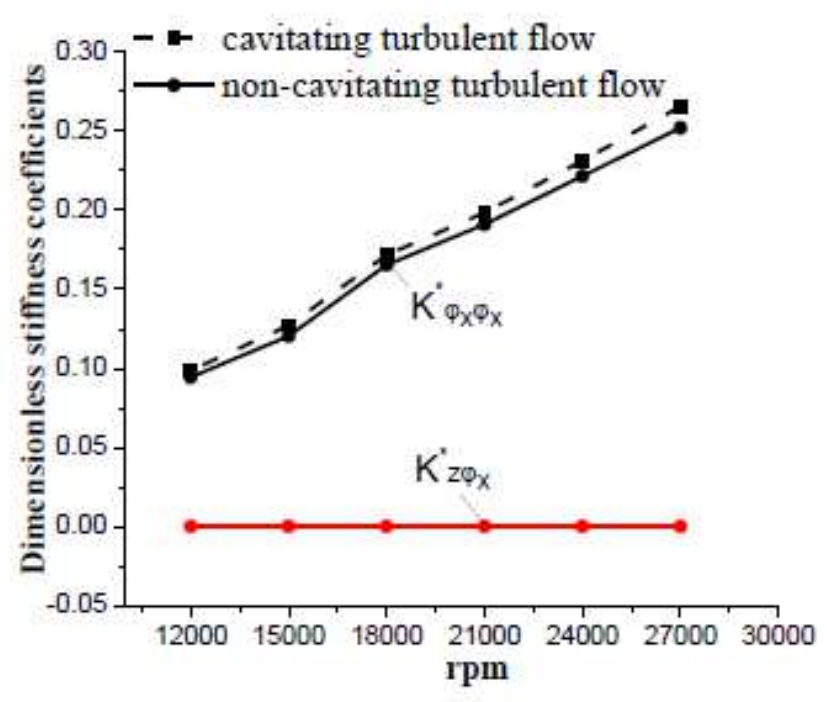

(c)

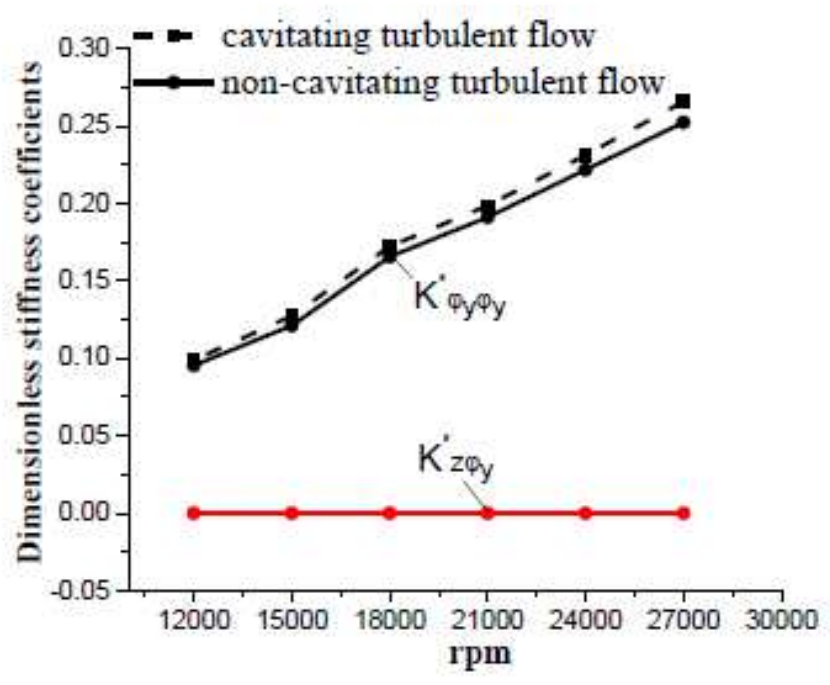

(b)

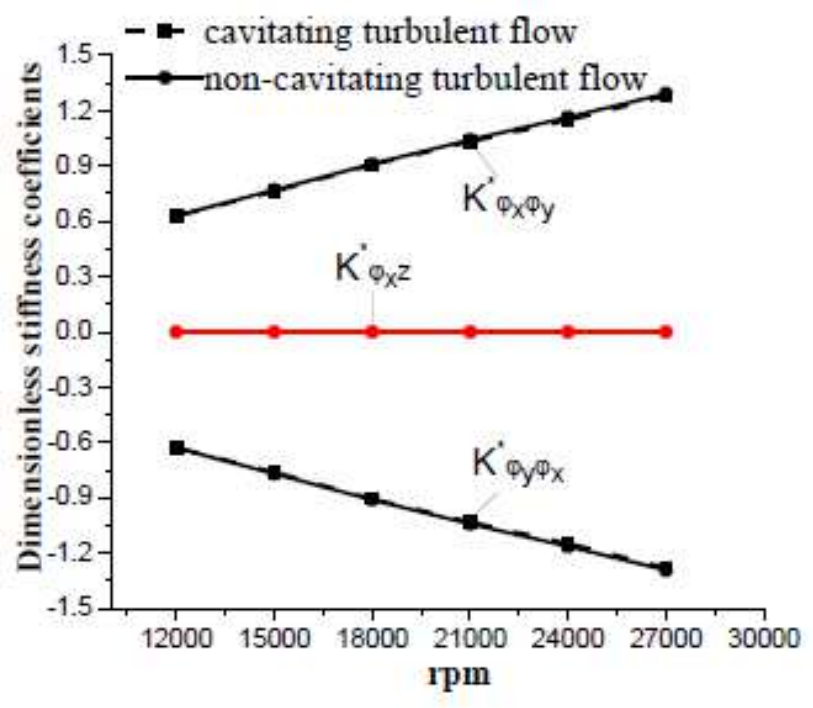

(d)

\section{Figure 11}

Influence of cavitation effect on stiffness coefficients for different rotating speed 


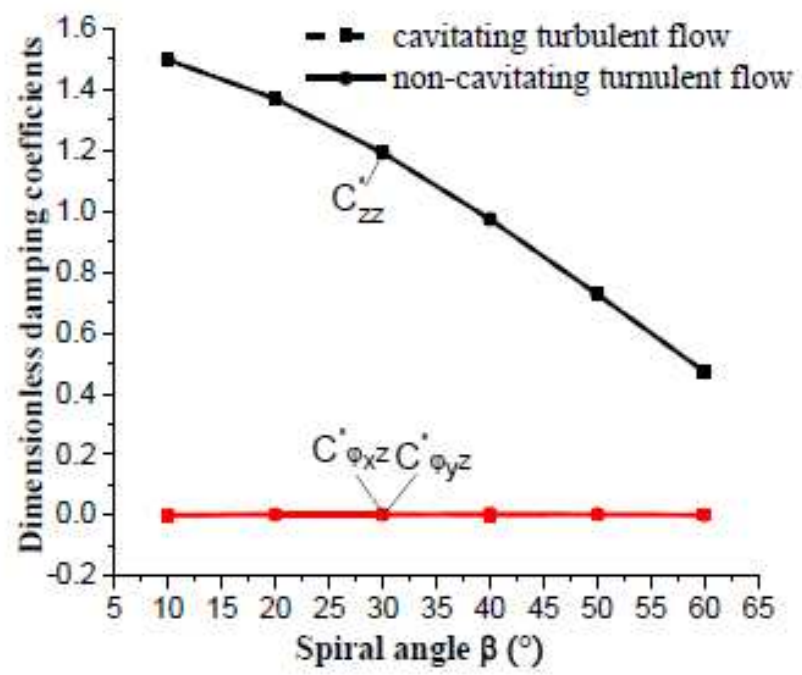

(a)

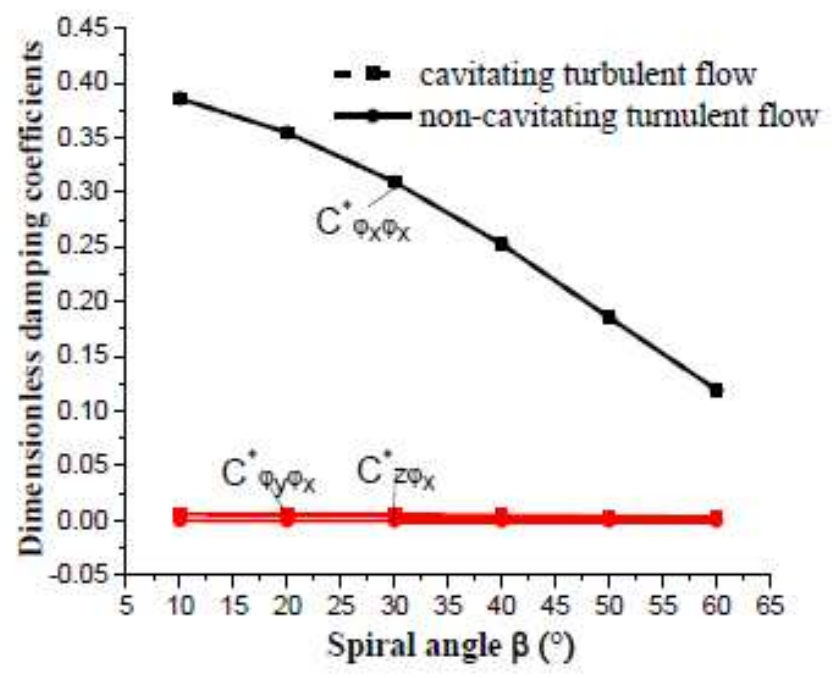

(b)

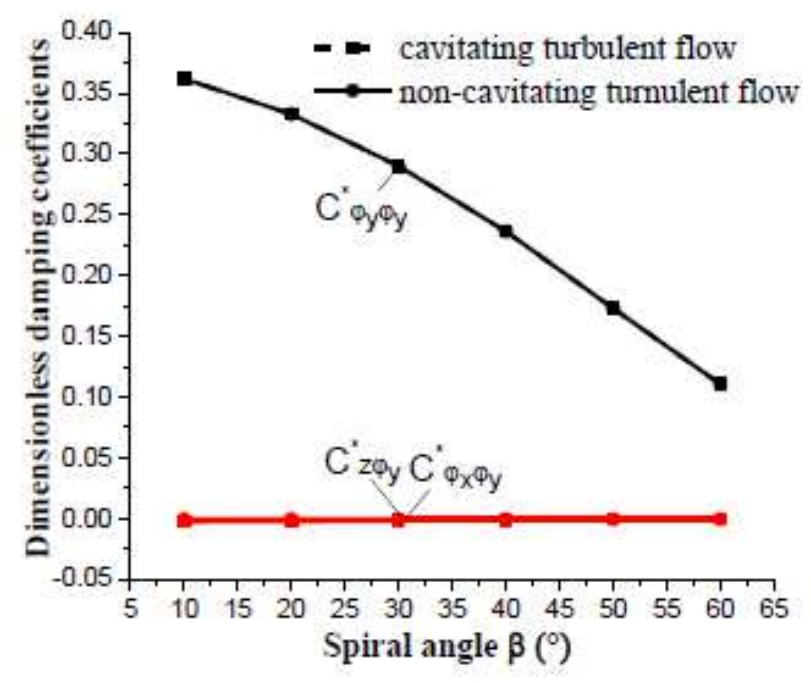

(c)

Figure 12

Influence of cavitation effect on the damping coefficients for different spiral angles 


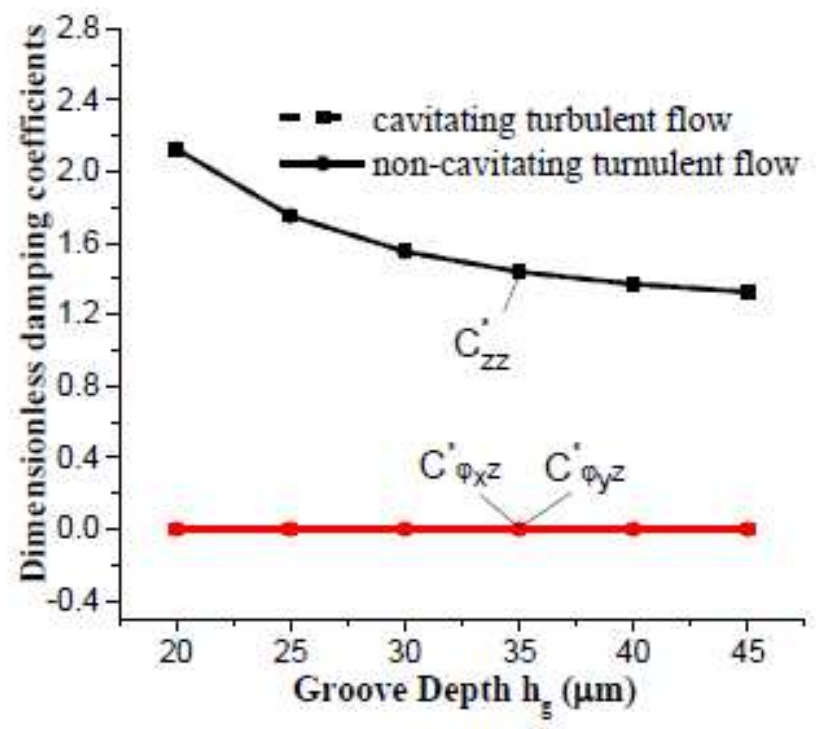

(a)

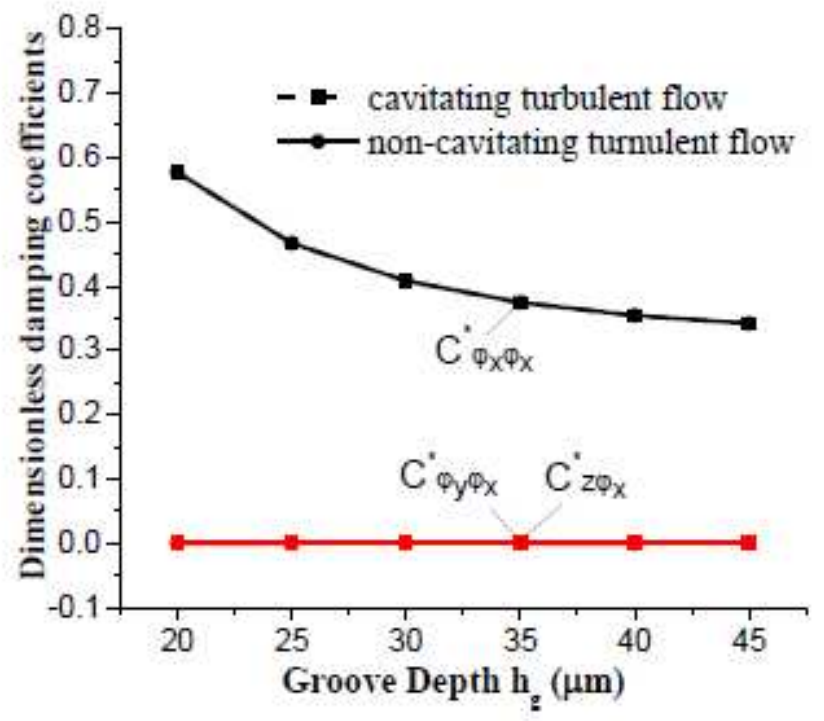

(b)

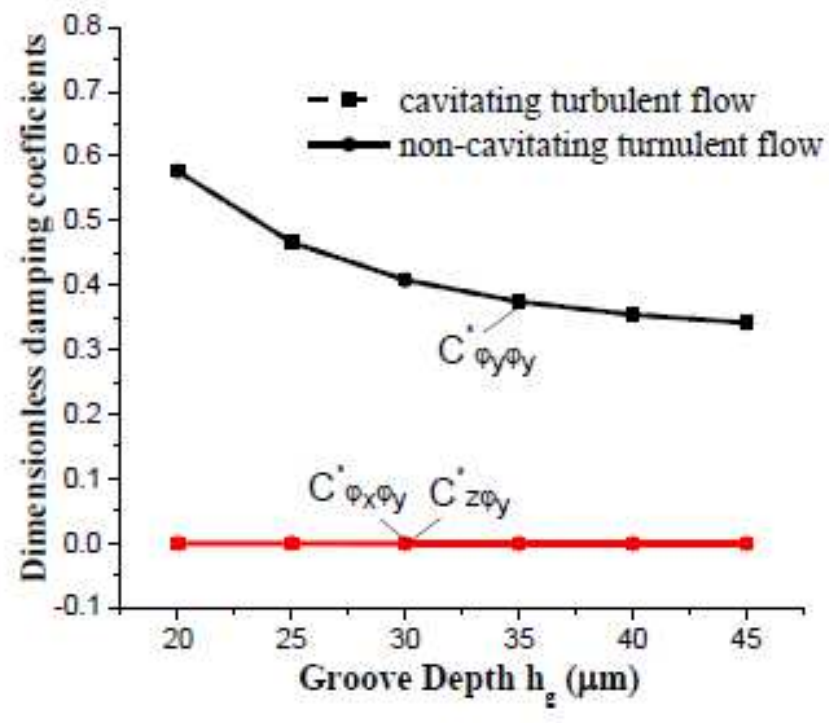

(c)

Figure 13

Influence of cavitation effect on damping coefficients for different groove depth. 


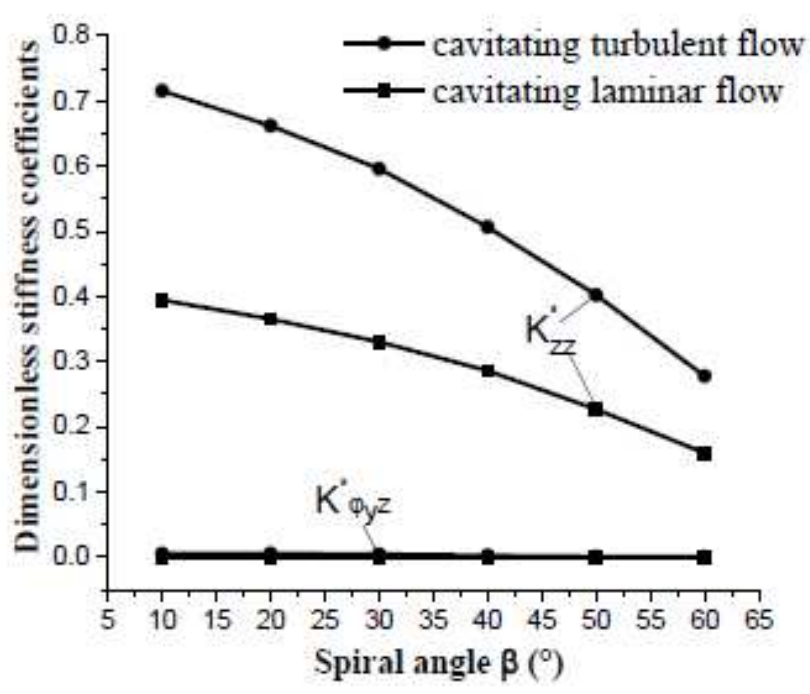

(a)

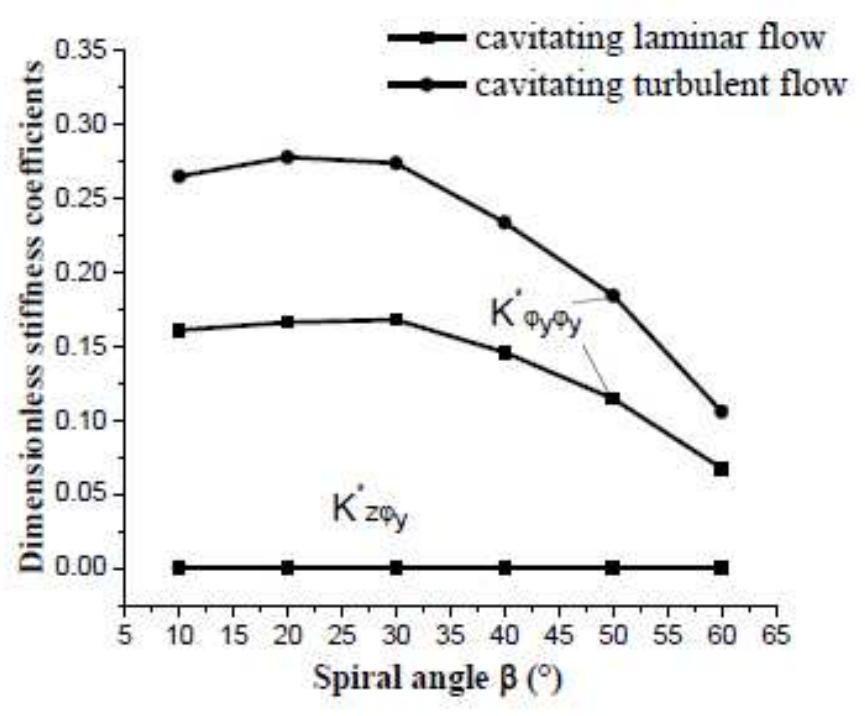

(c)

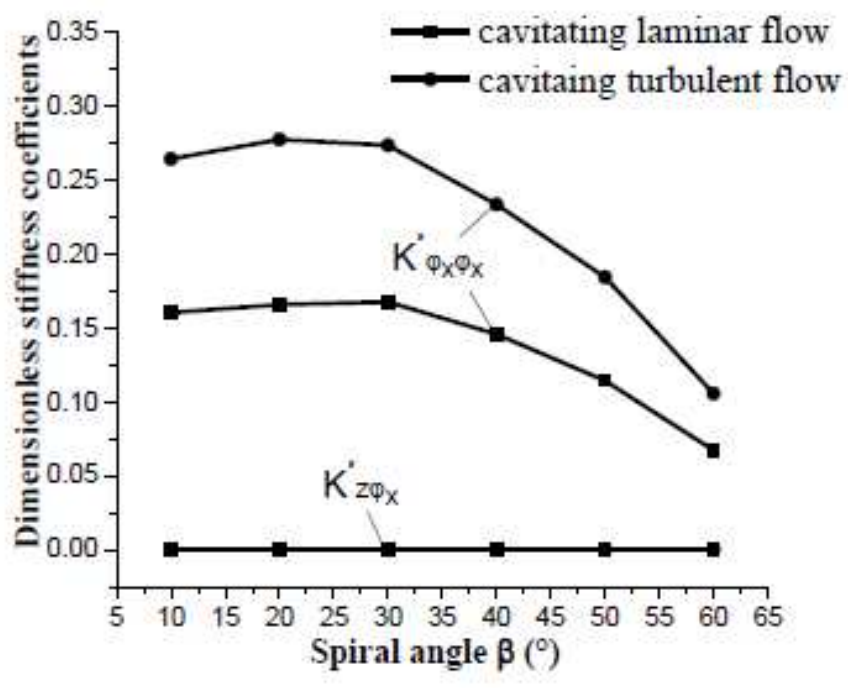

(b)

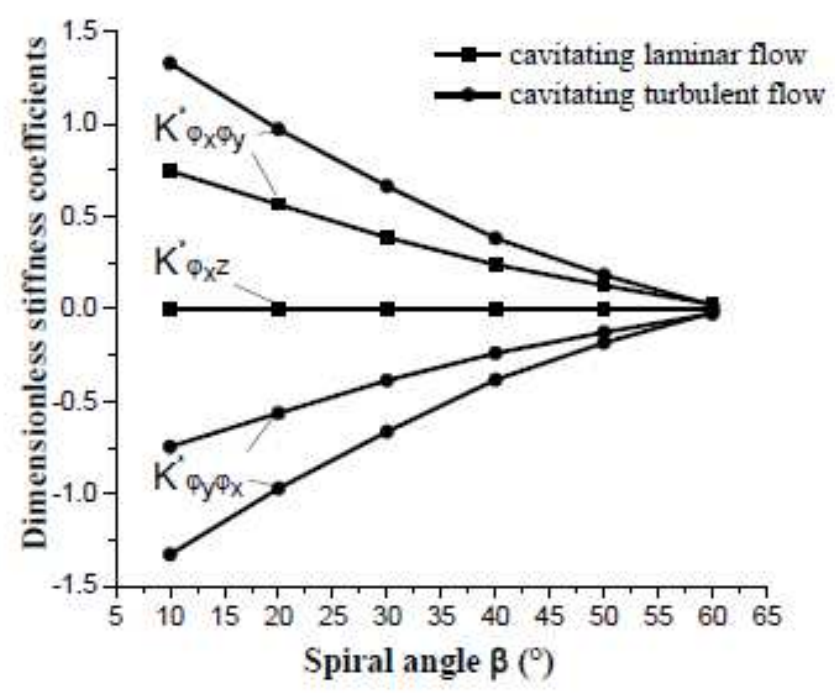

(d)

\section{Figure 14}

Influence of turbulence effect on the water film stiffness coefficient for different spiral angle 


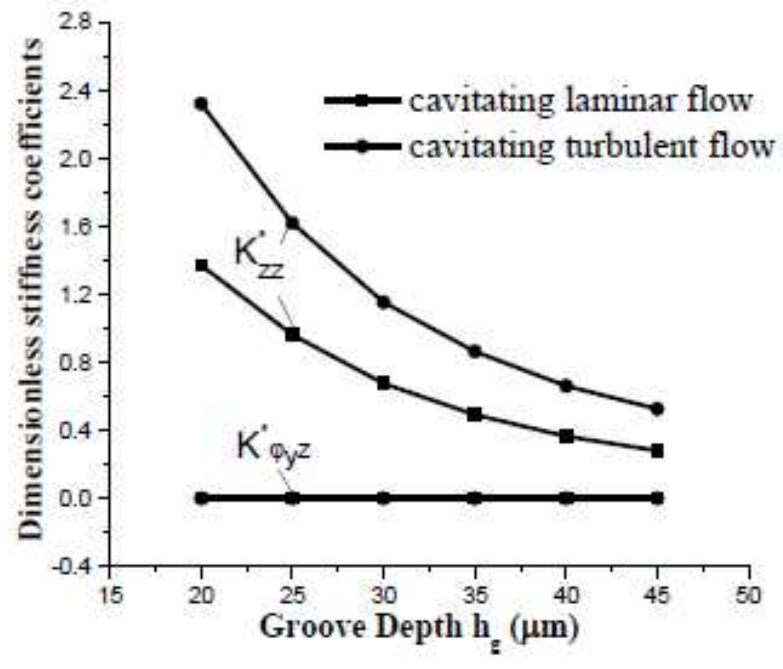

(a)

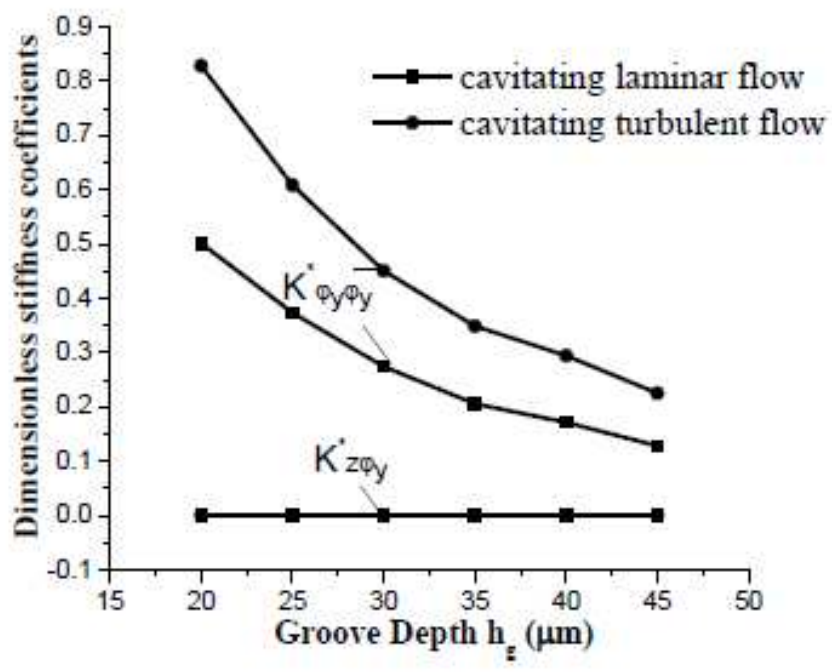

(c)

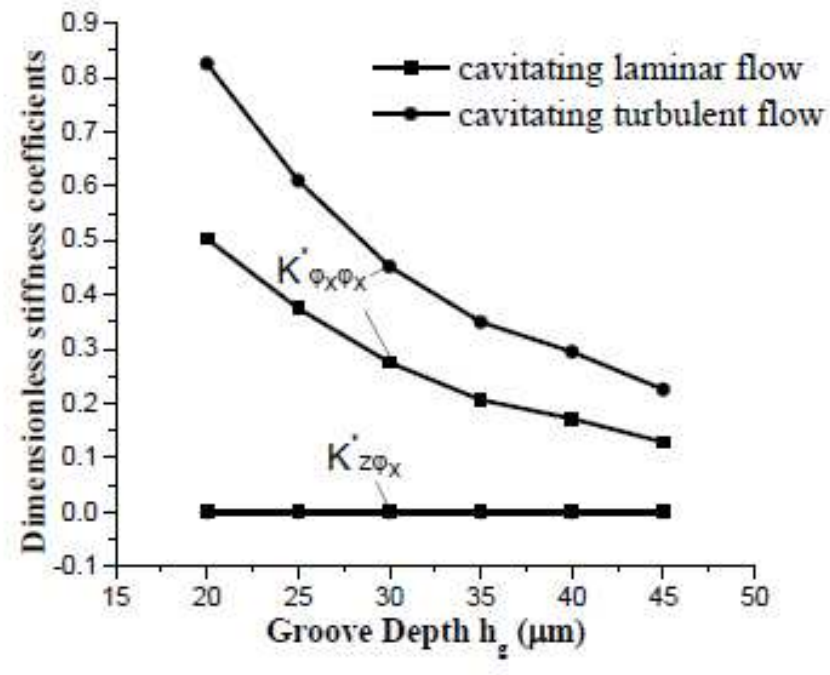

(b)

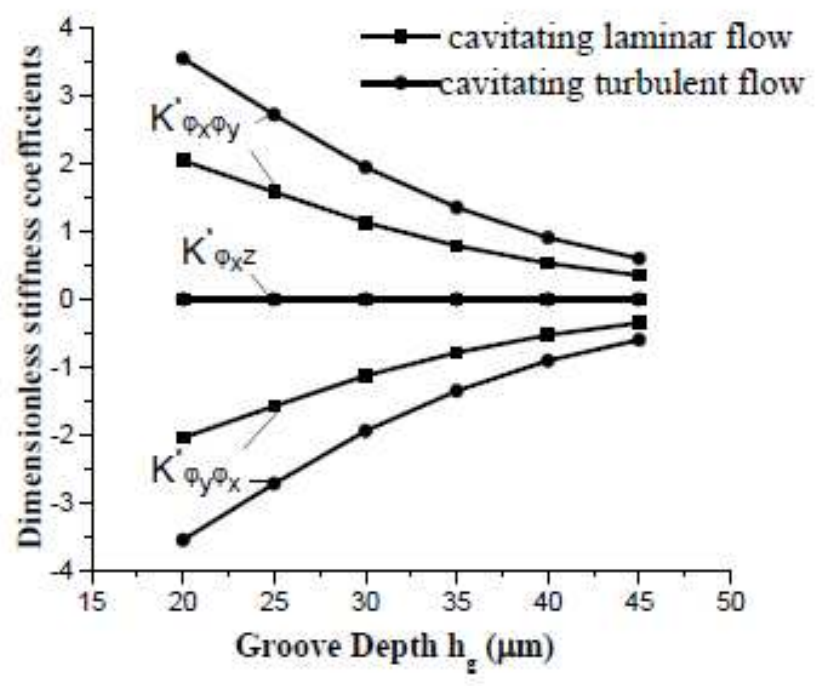

(d)

\section{Figure 15}

Influence of turbulence effect on the stiffness coefficient for different groove depths 


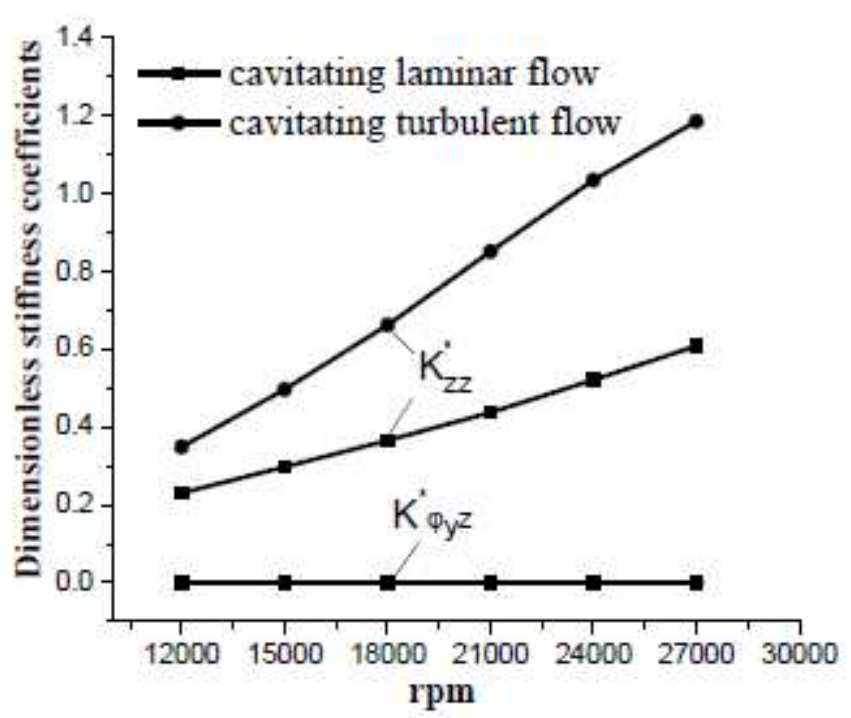

(a)

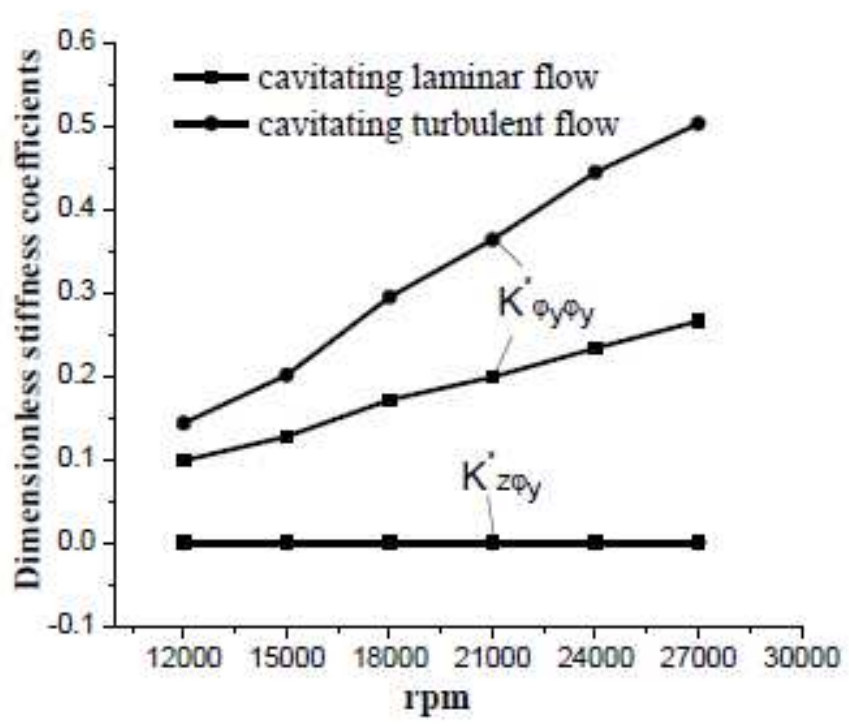

(c)

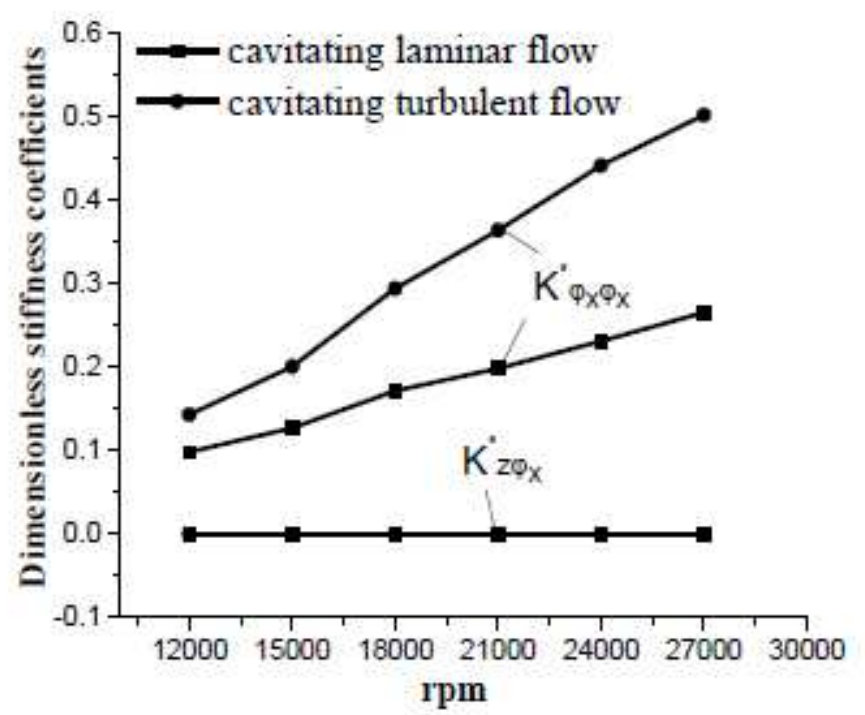

(b)

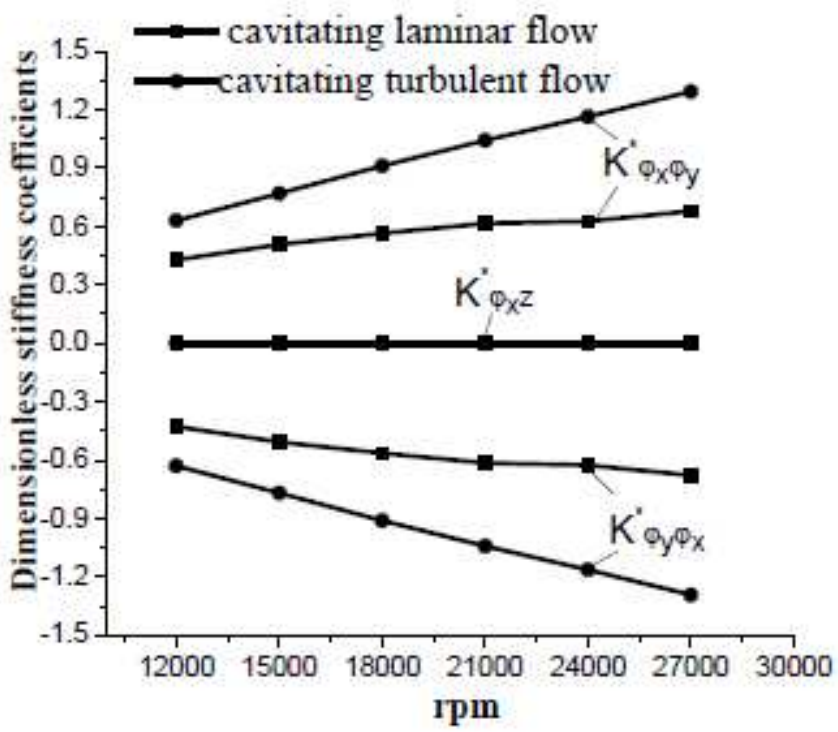

(d)

Figure 16

nfluence of turbulence effect on the stiffness coefficient for different otating speed 


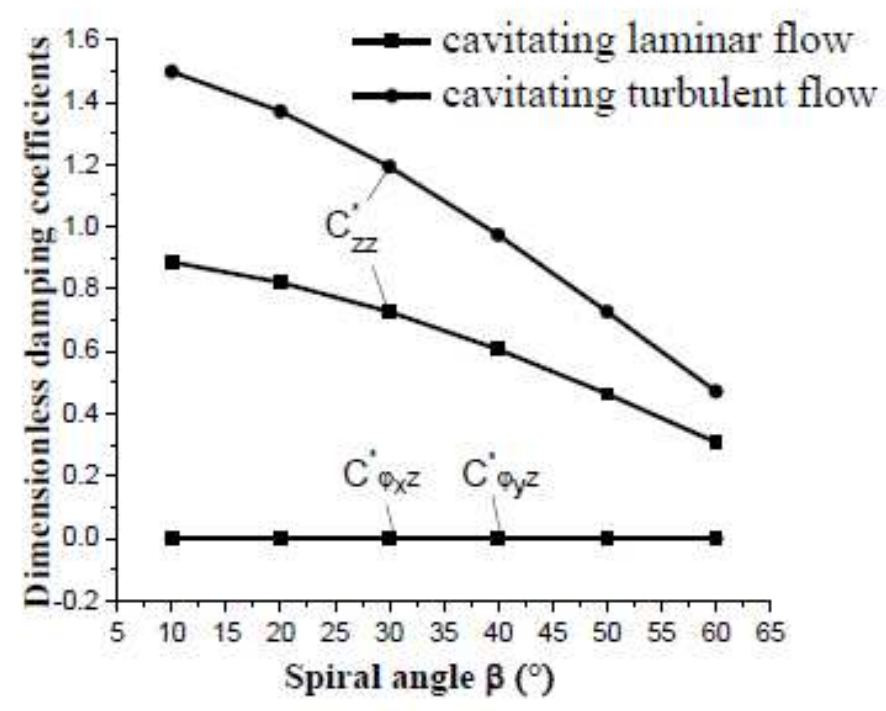

(a)

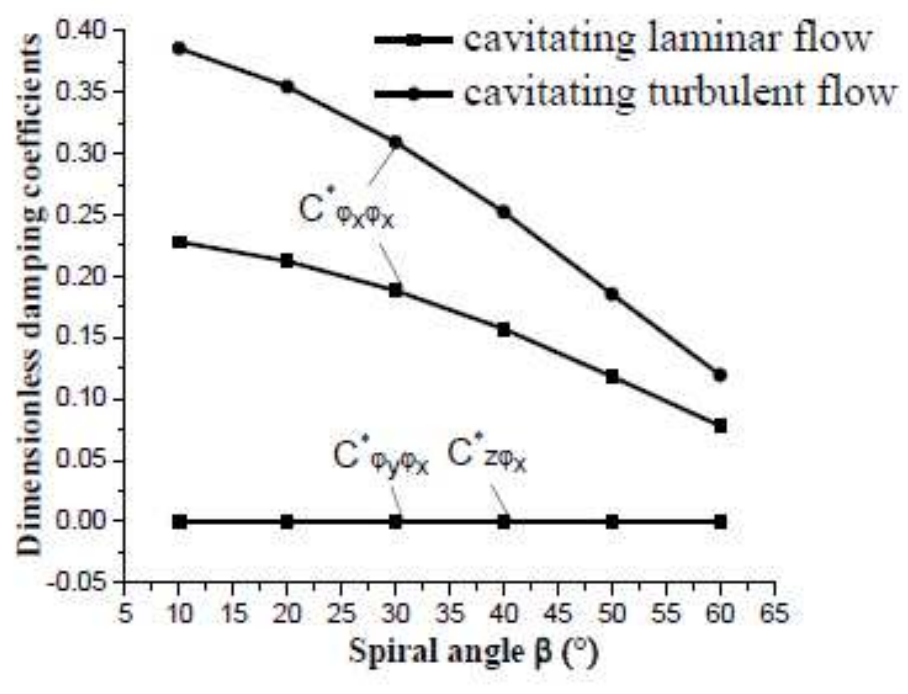

(b)

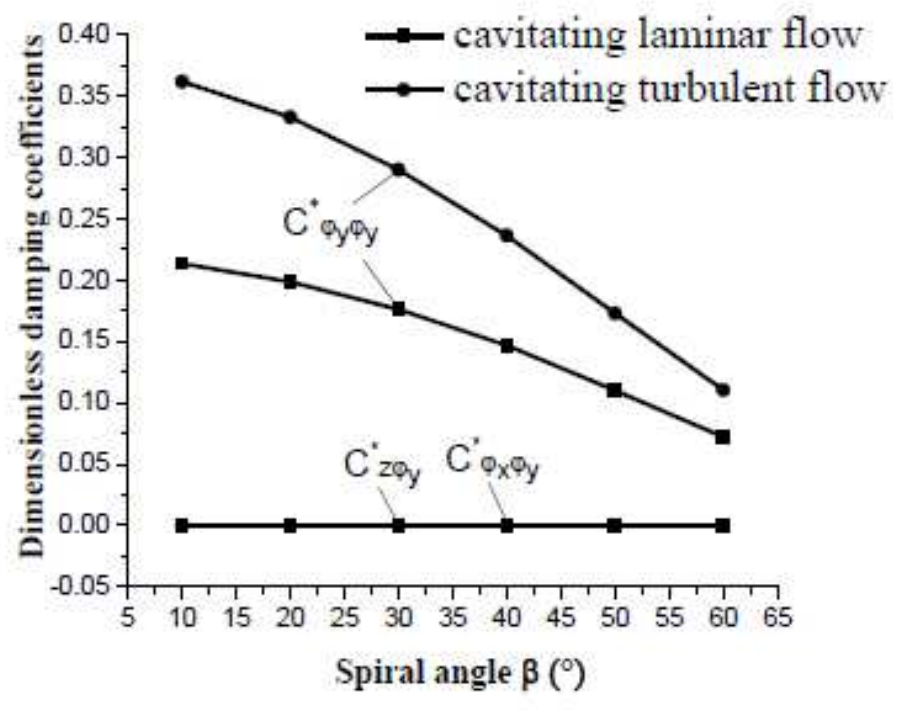

(c)

\section{Figure 17}

Influence of turbulence effect on the damping coefficients for different spiral angles. 

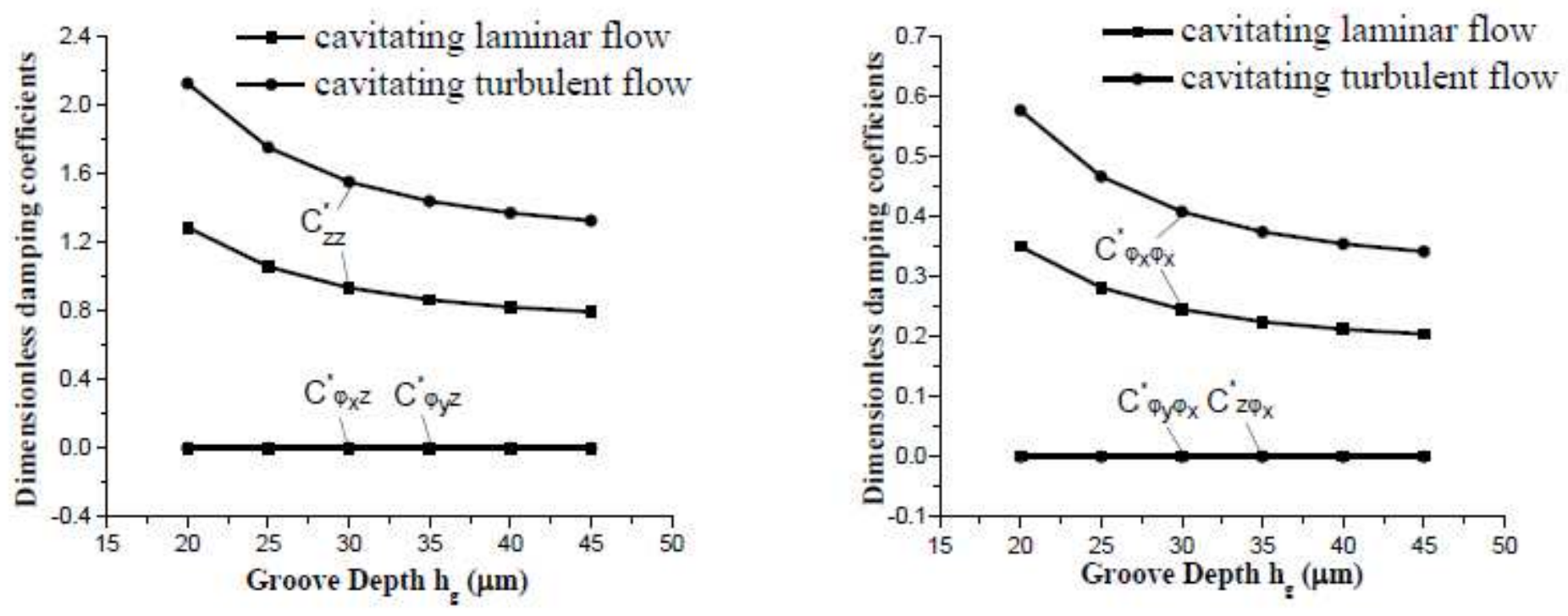

(a)

(b)

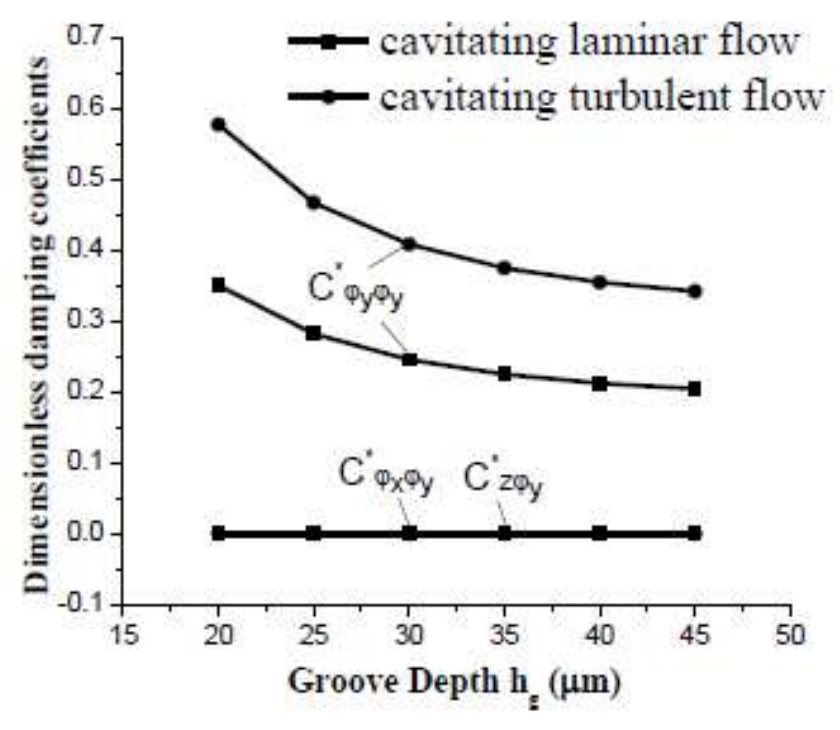

(c)

Figure 18

Influence of turbulence effect on the damping coefficients for different groove depths. 


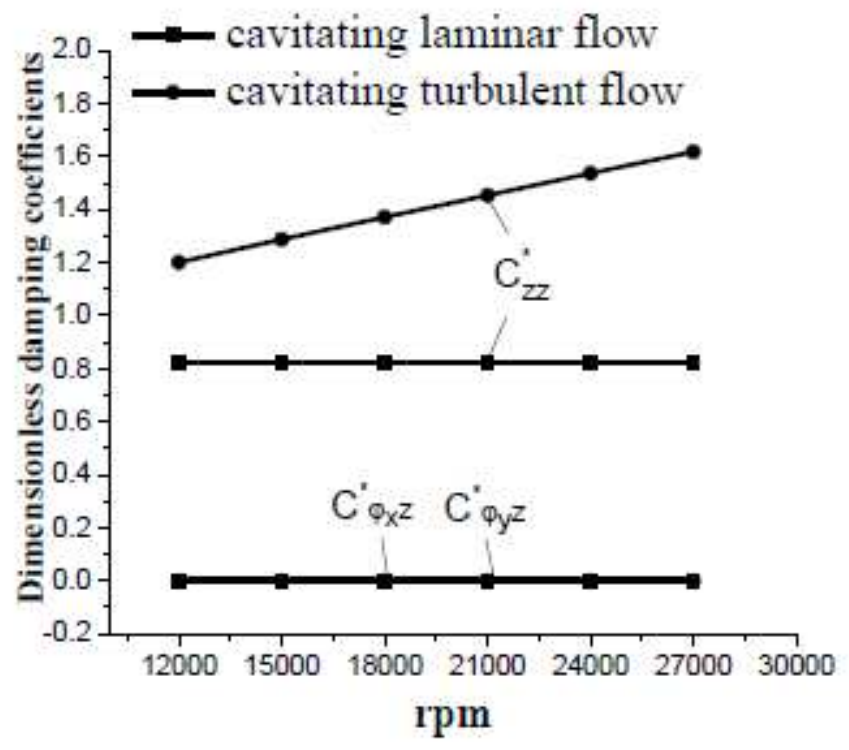

(a)

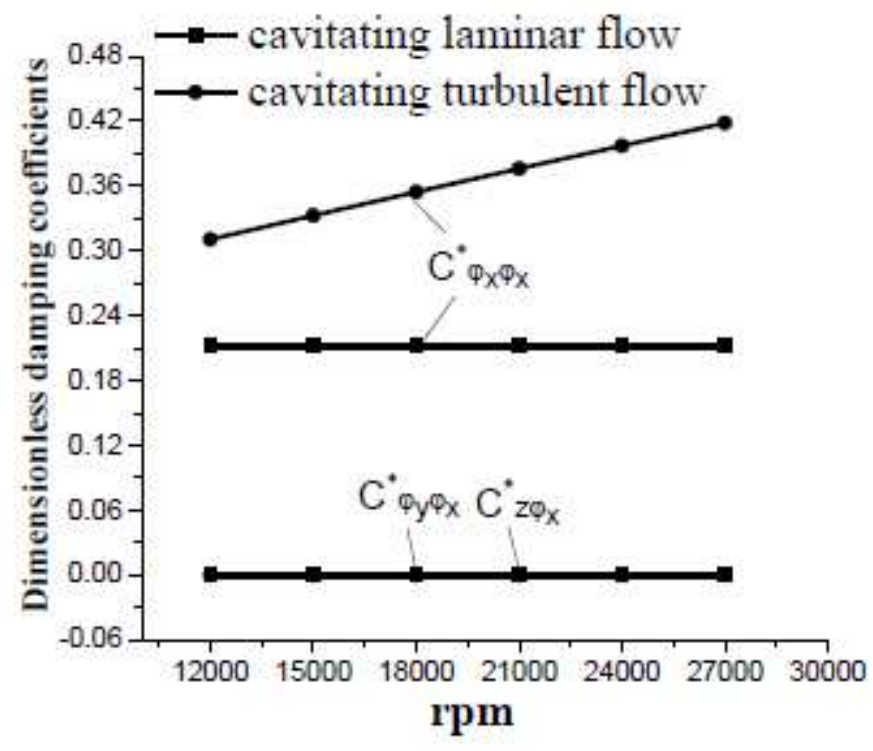

(b)

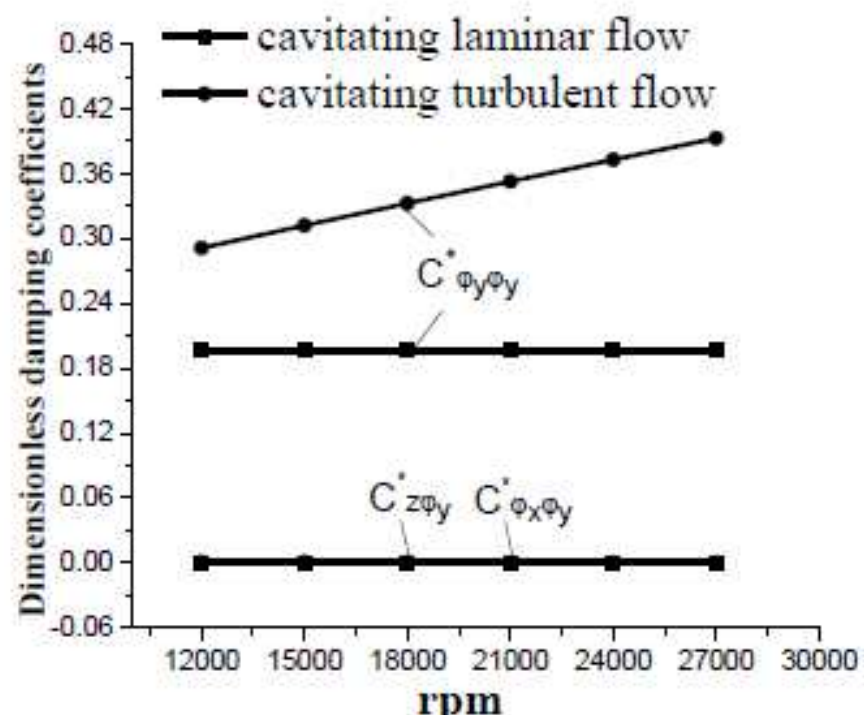

(c)

Figure 19

nfluence of turbulence effect on the damping coefficients for different rotating speed. 


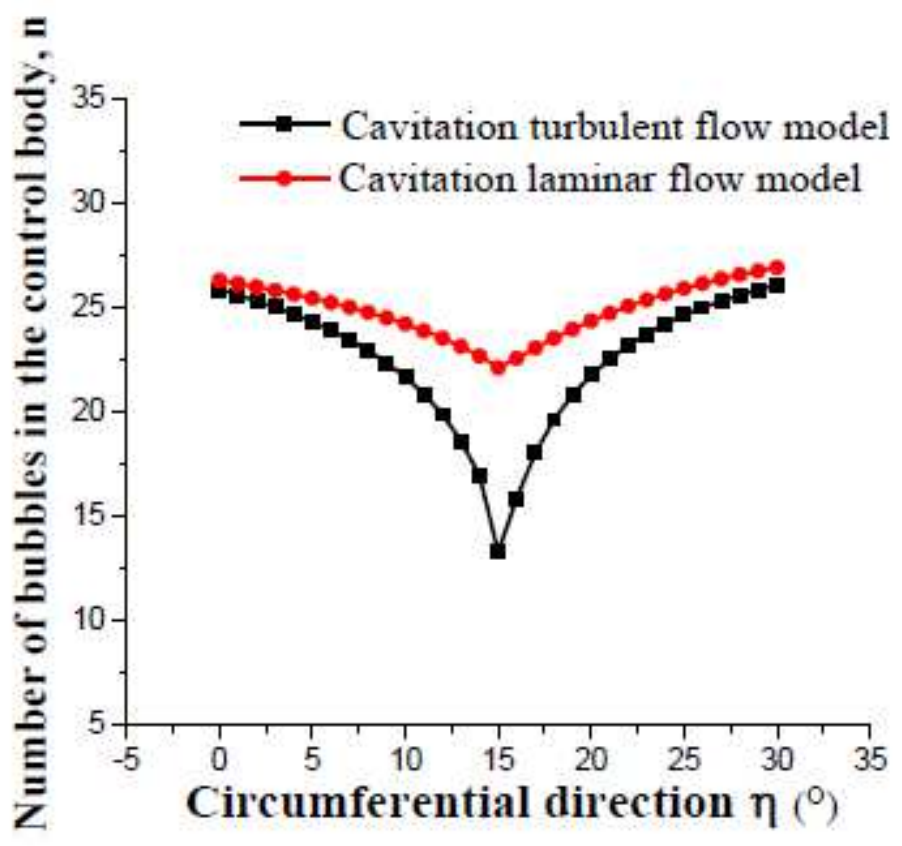

(a)

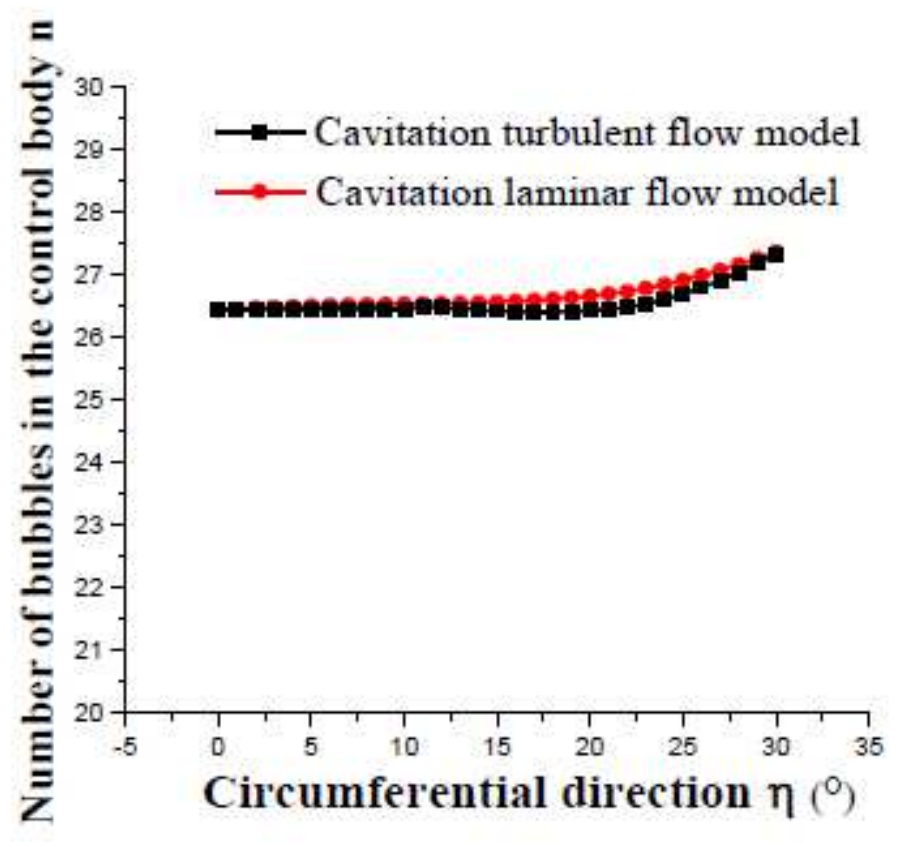

(b)

Figure 20

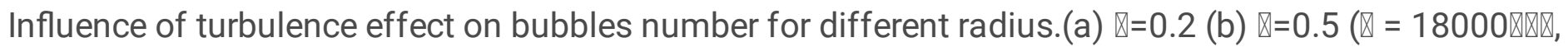
$\mathrm{Q}=20 \mathrm{Q}, \mathrm{Q} \mathrm{Q}=15 \mathrm{Q} \mathrm{Q}, \mathrm{Q} \mathrm{Q}=40 \mathrm{Q} \mathrm{Q}$ ) 